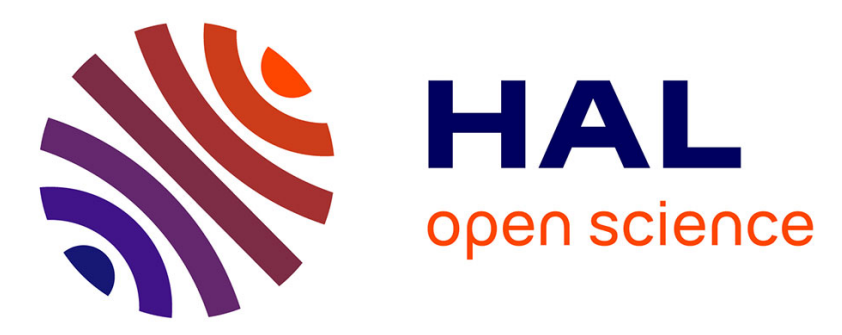

\title{
An introduction to the homogenization modeling of non-Newtonian and electrokinetic flows in porous media
} Andro Mikelic

\section{To cite this version:}

Andro Mikelic. An introduction to the homogenization modeling of non-Newtonian and electrokinetic flows in porous media. Springer. New trends in non-newtonian fluid mechanics and complex flow, 2212, Springer, pp.171-227, 2017, Lecture Notes Centro Internazionale Matematico Estivo (C.I.M.E.) Series, Lecture Notes in Mathematics, Springer. hal-01488276

\section{HAL Id: hal-01488276 https://hal.science/hal-01488276}

Submitted on 13 Mar 2017

HAL is a multi-disciplinary open access archive for the deposit and dissemination of scientific research documents, whether they are published or not. The documents may come from teaching and research institutions in France or abroad, or from public or private research centers.
L'archive ouverte pluridisciplinaire HAL, est destinée au dépôt et à la diffusion de documents scientifiques de niveau recherche, publiés ou non, émanant des établissements d'enseignement et de recherche français ou étrangers, des laboratoires publics ou privés. 


\title{
An introduction to the homogenization modeling of non-Newtonian and electrokinetic flows in porous media *
}

Andro Mikelić

\begin{abstract}
The flow of complex fluids through porous media is common to many engineering applications. The upscaling is a powerful tool for modeling nonhomogeneous media and we consider homogenization of quasi-Newtonian and electrokinetic flows through porous media. For the quasi-Newtonian polymeric fluids, the incompressible Navier-Stokes equations with the invariants dependent viscosity is supposed to hold the pore scale level. The 2-scale asymptotic expansions and the two-scale convergence of the monotone operators are applied to derive the reservoir level filtration law, given as a monotone relation between the filtration velocity and the pressure gradient. The second problem, we consider, is the quasi-static transport of an electrolyte through an electrically charged medium. The physical chemistry modeling is presented and used to get a dimensionless form of the problem. Next the equilibrium solutions are constructed through solving the Poisson-Boltzmann equation. For the solutions being close to the equilibrium, the two-scale convergence is applied to obtain the Onsager relations linking gradients of the pressure and of the chemical potentials to the filtration velocity and the ionic fluxes.
\end{abstract}

\footnotetext{
Andro Mikelić

Univ Lyon, Université Claude Bernard Lyon 1, CNRS UMR 5208,

Institut Camille Jordan, 43 blvd. du 11 novembre 1918,

F-69622 Villeurbanne cedex, France, e-mail: Andro.Mikelic@univ-lyon1.fr

* A chapter to appear in "New trends in non-newtonian fluid mechanics and complex flow", Lecture Notes Centro Internazionale Matematico Estivo (C.I.M.E.) Series, Lecture Notes in Mathematics, Springer, 2017. The corresponding lectures were given at the CIME-CISM Course "New trends in non-newtonian fluid mechanics and complex flows" held in Levico Terme, Italia, August 28- September 2, 2016,
} 


\section{Introduction to the homogenization}

Using the equations of the continuum physics at pore scale for porous media is a promising approach to derive the overall equations, but meets many difficulties. The presence of the fluid and the solid parts in the soil obliges us to consider it as a multiphase medium. The phases are geometrically present in a heterogeneous way, with small pores and cavities.

Homogenization applied to heterogeneous media (porous media, composites, tissus, etc) is a mathematical method that allows to "upscale" the fundamental equations from continuum physics, being valid at the microscopic level. Upscaling or homogenization signifies that the particular phases lose their independent presence in the model and will get "smeared". Rather than tracking behavior of every phase, we search to approximate the model with equations being valid everywhere. Phases get present in every point through new averaged unknowns like saturations and concentrations. This way it is not necessary to solve nonlinear PDEs of the fluid mechanics/elasticity/heat conduction in the complicated geometry of a heterogeneous medium. Note that, in addition, the pore geometry is usually unknown and avaible only through some statistical averages.

The homogenization theory of heterogeneous media studies the effects of the micro-structure (i.e. of the pore structure) upon solutions of PDEs of the continuum mechanics. Even in the simplest case of a viscous single phase flow through a porous medium, we are given a PDE with two natural length scales : a macroscopic scale (the scale of the piece of reservoir/soil) of order 1 and a microscopic scale (the pore scale or the scale of perforations) of order $\varepsilon$, the later measuring the scale of oscillations. This disparity in length scales is what provides us with our expansion parameter $\varepsilon$. For fixed, but small, characteristic pore length $\varepsilon>0$ the solutions $u^{\varepsilon}$ of the flow equations will in general be complicated, having different behaviors on the two length scales.

A closed-form solution is not achievable and a numerical solution would be nearly impossible to calculate. In the practical simulations of the flows through porous media, we use PDEs at the macroscale. Information about the pore structure is only kept through some averaged quantities as porosity and permeability.

Therefore, one of the fundamental questions in the modeling of flows through porous media is how to get the "averaged" or "upscaled " equations. Next we wish to calculate the effective coefficients describing the influence of the microstructure. Finally, it is of interest to know whether our derived model is correct, in the sense that it should approximate the original problem involving the micro-structure.

In the homogenization theory, the upscaling corresponds to the study of the limiting behavior $u^{\varepsilon} \rightarrow u$ as $\varepsilon \rightarrow 0$. The idea is that in this limit the micro-structure (generating the high-frequency oscillations) will " average out", and there will be a simple " averaged " or " homogenized" PDE, which will represent a filtration law.

As even the simple example of Darcy's law confirms, the homogenized PDE can differ much from the original one. In overcoming this fundamental difficulty it is 
useful to use formal multiscale expansions in $\varepsilon$, containing behavior on different length scales.

The idea is to suppose $u^{\varepsilon}$ has the following expansion:

$$
u^{\varepsilon}=\varepsilon^{\beta}\left\{u_{0}\left(x, \frac{x}{\varepsilon}\right)+\varepsilon u_{1}\left(x, \frac{x}{\varepsilon}\right)+\varepsilon^{2} u_{2}\left(x, \frac{x}{\varepsilon}\right)+\ldots\right\}
$$

Two-scale expansion (1) is plugged into the PDE and we search for a scale of equations determining the functions $u_{i}, i=1, \ldots$ Nevertheless, before plugging expansion (1) into the PDE, we should somehow determine $\beta$.

In order to answer all those questions, we establish the following strategy, which we are going to apply in the sections which follow:

A) A description of the geometry of the heterogeneous medium is given. It can be periodic, statistically homogeneous etc.

B) A continuum physics model valid at the pore scale is written up. The model can come either from the well-established textbook modeling or from the molecular dynamics calculations allowing to go from the molecular structure to the continuum mechanics at micro/nano-metric scale.

C) The a priori estimates for solutions of the PDE, uniform with respect to $\varepsilon$, are established. For the flow problems we usually need:

C1) A priori estimates for the velocity.

C2) A priori estimates for the pressure.

D) Having obtained a priori estimates, a formal multiscale expansion is set up in the form (1). We shall see that for the linear and monotone problems it corresponds to passing to the homogenization limit in the sense of the 2-scale convergence.

E) The upscaled problem is studied. We prove uniqueness and regularity and undertake separation of the fast and slow scales. A numerical method for calculating the effective coefficients is proposed.

This short chapter will try to initiate the reader to the applications of the 2-scale convergence technique in the homogenization of complex flow through porous media. We present 3 examples of complex flows through a porous medium: the 1 st is the homogenization of a quasi-Newtonian flow, the 2 nd is the homogenization of a Bingham flow and the 3rd is the derivation of the Onsager relations for the electrokinetic flows.

In connection with the homogenization in porous media, we recommend to the reader the book edited by U. Hornung [39]. It contains number of contributed chapters, and we mention the chapters on the 2-scale convergence and on the derivation of Darcy's law by homogenization by G. Allaire, which we are going to quote frequently in this text.

As general references on homogenization we recommend the classic text by E. Sanchez-Palencia [72] and the recent engineering textbook by Mei and Vernescu 
[53]. More recent mathematical references are the books by Jikov, Kozlov and Oleinik [40], Cioranescu and Donato [29] and Pavliotis [67].

Classical references on 2-scale convergence are papers by G. Allaire [5] and by G. Nguetseng [63].

\section{Models for quasi-Newtonian fluids and a derivation of the filtration laws by a two-scale expansion}

In this section we first present models of quasi-Newtonian fluids. Then we discuss their well-posedness and particularities of the geometry. After obtaining a priori estimates, we propose two-scale expansions. They allow achieving our goal of deriving formally equations describing filtration of a quasi-Newtonian fluid.

\subsection{Continuum physics models for quasi-Newtonian fluids}

We first recall the fluid mechanics equations at the pore level.

The incompressible quasi-Newtonian fluids are characterized by the viscosity depending on the principal invariants of the symmetric stretching tensor $D(\mathbf{v})$. In our notation, $\mathbf{v}$ is the velocity, $p$ the pressure, $\nabla \mathbf{v}$ the gradient velocity tensor and $D(\mathbf{v})=\left(\nabla \mathbf{v}+\nabla \mathbf{v}^{t}\right) / 2$ will denote the rate-of-strain or the symmetric stretching $)$ tensor. The principal invariants of $D(\mathbf{v})$ are

$$
I_{1}(D)=\operatorname{tr} D=\operatorname{div} \mathbf{v}, \quad I_{2}(D)=\frac{1}{2}\left((\operatorname{div} \mathbf{v})^{2}-\operatorname{tr} D^{2}\right) \quad \text { and } \quad I_{3}(D)=\operatorname{det} D .
$$

$\sigma$ is the stress tensor $\sigma=-p I+2 \eta_{r} D(\mathbf{v})$. The viscosity $\eta_{r}$ is constant for a Newtonian fluid but dependent on the shear rate $\dot{\gamma}=\sqrt{|D(\mathbf{v})|^{2} / 2}=\sqrt{I_{2}(D(\mathbf{v})}$, i.e. $\eta_{r}=\eta_{r}(\dot{\gamma})$, for viscous non-Newtonian fluids. The deviatoric stress tensor $\tau$, i.e. the part of the total stress tensor that is zero at equilibrium, is then a nonlinear function of the symmetric stretching tensor $D(\mathbf{v})$,

$$
\tau=\eta_{r}(\dot{\gamma}) D(\mathbf{v})
$$

Two most widely used laws in engineering practice are the power law and the Carreau-Yasuda law.

The first most popular empiricism is the "power law" or Ostwald-de Waele model, where the expression for the shear rate dependent viscosity is

$$
\eta_{r}(\xi)=\lambda_{0}|\xi|^{r-2}=\lambda_{1}|\dot{\gamma}|^{r-2}=\lambda_{1}|\dot{\gamma}|^{n-1}, \quad \xi \in \mathbb{R}^{9}
$$


where $n=r-1$ is the power-law exponent and $\lambda_{1}$ is the consistency of the fluid. For $1<r<2$ the fluid is a shear thinning and for $r>2$ a shear-thickening.

The simple power-law model (equation(2)) has a well-known singularity at zero shear rate, which must be carefully accounted for in kinematic analyses. The Carreau-Yasuda equation is an alternate generalized Newtonian model that enables the description of the plateaus in viscosity that are expected when the shear rate is very small or very large. The empiricism for the viscosity $\eta_{r}$ used in the CarreauYasuda law is

$$
\eta_{r}(\xi)=\left(\eta_{0}-\eta_{\infty}\right)\left(1+\lambda|\xi|^{2}\right)^{r / 2-1}+\eta_{\infty}, \quad \xi \in \mathbb{R}^{9},
$$

with $\eta_{0} \geq \eta_{\infty}>0, \lambda>0$, where $\eta_{0}$ is the zero-shear-rate viscosity, $\eta_{\infty}$ is the infiniteshear-rate viscosity, $\lambda$ is a time constant being the inverse of a characteristic shear rate at which shear thinning becomes important and $r-1$ is a dimensionless constant describing the slope in the "power law region" of $\log \eta_{r}$ versus $\log \dot{\gamma}$.

The incompressible quasi-Newtonian Navier-Stokes system is given by

$$
\begin{array}{cc}
-\nabla \cdot\left\{\eta_{r}(\dot{\gamma}) D(\mathbf{v})\right\}+\rho(\mathbf{v} \nabla) \mathbf{v}+\nabla p=\rho \mathbf{f} & \text { in } \Omega_{p}, \\
\nabla \cdot \mathbf{v}=0 & \text { in } \Omega_{p}, \\
\mathbf{v}=0 & \text { on } \partial \Omega_{p},
\end{array}
$$

where $\Omega_{p}$ is the pore space of the porous medium.

The corresponding functional space for the velocity is

$$
V_{r}\left(\Omega_{p}\right)=\left\{\mathbf{z} \in W_{0}^{1, r}\left(\Omega_{p}\right)^{3}: \nabla \cdot \mathbf{z}=0 \text { in } \Omega_{p}\right\},
$$

where $1<r<+\infty$ and $W_{0}^{1, r}\left(\Omega_{p}\right)=\left\{z \in L^{r}\left(\Omega_{p}\right) \mid \nabla z \in L^{r}\left(\Omega_{p}\right)^{3}\right\} . \Omega_{p}$ is a bounded open set with a smooth boundary and $\mathbf{f}$ is a smooth function.

In two and three dimensions the classical theory from Lions, Cattabriga and Temam (see [44], [26] and [80]), and newly developed techniques from [25] give the existence of at least one weak solution $(\mathbf{v}, p) \in V_{r}\left(\Omega_{p}\right) \times L_{0}^{r^{\prime}}\left(\Omega_{p}\right)$ for (2),(4)-(6) (i.e. the power law) under assumption, $r>2 d /(d+2)$ (i.e. $r>1$ in the two dimensional case and $r>6 / 5$ in three dimension), with $r^{\prime}=r /(r-1)$. For system (3)-(6) (i.e. for the case of Carreau-Yasuda law) we have existence of at least one weak solution $(\mathbf{v}, p) \in V_{2}\left(\Omega_{p}\right) \times L_{0}^{2}\left(\Omega_{p}\right)$ for $1<r \leq 2$ and $(\mathbf{v}, p) \in V_{r}\left(\Omega_{p}\right) \times L_{0}^{r^{\prime}}\left(\Omega_{p}\right)$ for $r>2$.

In order to make modeling more precise, we define the dimensionless geometrical structure of the porous medium. We will divide $\Omega_{p}$, which is a subset of $\Omega=(0, L)^{3}$, by the characteristic length $L_{0}$ and obtain $\Omega_{\varepsilon}$. 


\subsection{The geometry of a periodic porous medium and a priori estimates}

Now we consider a periodic porous medium $\Omega=\left(0, L / L_{0}\right)^{3}$ in $\mathbb{R}^{3}$ with a periodic arrangement of the pores. The formal description goes along the following lines: First we define the geometrical structure inside the unit cell $Y=(0,1)^{3}$. Let $Y_{s}$ (the solid part) be a closed subset of $\bar{Y}$ and $Y_{F}=Y \backslash Y_{S}$ (the fluid part). The liquid/solid interface is $S=\partial Y_{S} \backslash \partial Y$. See Fig. 1 for a typical unit cell.

We make the periodic repetition of $Y_{s}$ all over $\mathbb{R}^{3}$ and set $Y_{s}^{k}=Y_{s}+k, k \in \mathbb{Z}^{3}$. Obviously the set $E_{S}=\bigcup_{k \in \mathbb{Z}^{3}} Y_{s}^{k}$ is a closed subset of $\mathbb{R}^{3}$ and $E_{F}=\mathbb{R}^{3} \backslash E_{S}$ is an open set in $\mathbb{R}^{3}$. The following assumptions on $Y_{F}$ and $E_{F}$ have been made:

(i) $Y_{F}$ is an open connected set of strictly positive measure, with a Lipschitz boundary and $Y_{S}$ has strictly positive measure in $\bar{Y}$ as well.

(ii) $E_{F}$ and the interior of $E_{S}$ are open sets with the boundary of class $C^{1,1}$, which are locally located on one side of their boundary. Moreover $E_{F}$ is connected and the solid part, $E_{S}$, is supposed connected in $\mathbb{R}^{3}$ (in two dimensions this hypothesis is not realistic).

Now we see that $\Omega=\left(0, L / L_{0}\right)^{3}$ is covered with a regular mesh of size $\varepsilon$, each cell being a cube $Y_{i}^{\varepsilon}=\varepsilon(Y+i)$, with $1 \leq i \leq N(\varepsilon)=|\Omega| \varepsilon^{-3}[1+0(1)]$. We define $Y_{s_{i}}^{\varepsilon}=\left(\Pi_{i}^{\varepsilon}\right)^{-1}\left(Y_{s}\right)$ and $Y_{f_{i}}^{\varepsilon}=\left(\Pi_{i}^{\varepsilon}\right)^{-1}\left(Y_{F}\right)$. For sufficiently small $\varepsilon>0$ we consider $T_{\varepsilon}=\left\{k \in \mathbb{Z}^{3} \mid Y_{s_{k}}^{\varepsilon} \subset \Omega\right\}$ and define

$$
\Omega_{s}^{\varepsilon}=\bigcup_{k \in T_{\varepsilon}} Y_{s_{k}}^{\varepsilon}, \quad \Gamma^{\varepsilon}=\partial \Omega_{s}^{\varepsilon}, \quad \Omega_{\varepsilon}=\Omega \backslash \Omega_{s}^{\varepsilon} .
$$

Obviously, $\partial \Omega_{\varepsilon}=\partial \Omega \cup \Gamma^{\varepsilon}$. The domains $\Omega_{s}^{\varepsilon}$ and $\Omega_{\varepsilon}$ represent, respectively, the solid and fluid parts of a porous medium $\Omega$.

For simplicity, we will suppose that $L /\left(L_{0} \varepsilon\right)$ is an integer.

Remark 1. A two-dimensional porous medium could be seen as a section of a bundle of parallel fibers. Possible geometries are shown on figures 2.

The no-slip condition on the pore boundaries is at the origin of velocity oscillations. They are precisely described by the following, pore-size dependent, Poincaré's inequality in a porous medium

Lemma 1. (see [72]) For each $\mathbf{w} \in W_{0}^{1, q}\left(\Omega_{\varepsilon}\right)^{3}$, the inequality

$$
\|\mathbf{w}\|_{L^{q}\left(\Omega_{\varepsilon}\right)^{3}} \leq C \varepsilon\|D(\mathbf{w})\|_{L^{q}\left(\Omega_{\varepsilon}\right)^{9}}, \quad 1<q<+\infty,
$$

holds true.

The equivalence between the norms $\|D(\mathbf{w})\|_{L^{q}\left(\Omega_{\varepsilon}\right)^{9}}$ and $\|\nabla \mathbf{w}\|_{L^{q}\left(\Omega_{\varepsilon}\right)^{9}}$ is due to Korn's inequality in porous media 


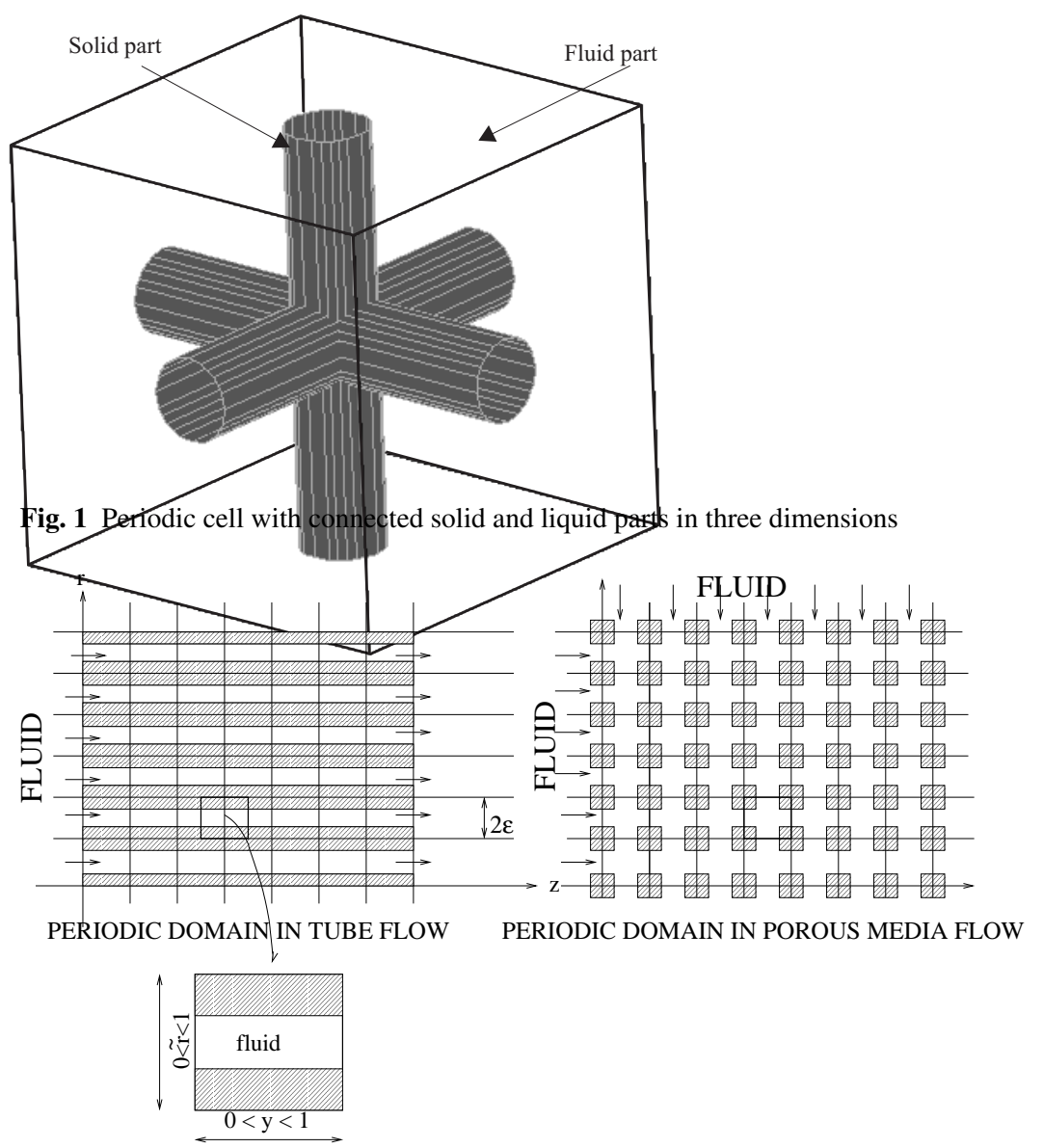

Fig. 2 Examples of two-dimensional porous media

Proposition 1. (see [65] and references therein) For each $\mathbf{w} \in W_{0}^{1, q}\left(\Omega_{\varepsilon}\right)^{3}, 1<q<$ $+\infty$, we have the inequality

$$
\|\nabla \mathbf{w}\|_{L^{q}\left(\Omega_{\varepsilon}\right)^{9}} \leq C\|D(\mathbf{w})\|_{L^{q}\left(\Omega_{\varepsilon}\right)^{9}} .
$$

Next we test equation (4) with the solution and obtain

$$
\|\mathbf{v}\|_{L^{r}}+\varepsilon\|D(\mathbf{v})\|_{L^{r}} \leq C \varepsilon^{r /(r-1)}
$$


in the case of the power law and

$$
\|\mathbf{v}\|_{L^{r}}+\varepsilon\|D(\mathbf{v})\|_{L^{r}} \leq C \varepsilon^{\beta}, \quad \beta=\min \{r /(r-1), 2\}
$$

in the case of the Carreau-Yosuda law.

Hence the characteristic velocity $U$ is of order $O\left(\varepsilon^{\beta}\right)$ and the Reynolds number $\operatorname{Re}=\rho L_{0} U / \eta_{0}$ is small. Therefore, it is enough to consider the quasi-Newtonian Stokes equations.

Consequently, in the case of power law (2) we consider the dimensionless system

$$
\begin{array}{cc}
-\nabla \cdot\left\{\left|D\left(\mathbf{v}^{\varepsilon}\right)\right|^{r-2} D\left(\mathbf{v}^{\varepsilon}\right)\right\}+\nabla p^{\varepsilon}=\mathbf{f} & \text { in } \Omega_{\varepsilon}, \\
\nabla \cdot \mathbf{v}^{\varepsilon}=0 & \text { in } \Omega_{\varepsilon}, \\
\mathbf{v}^{\varepsilon}=0 \quad \text { on } \partial \Omega_{\varepsilon} . &
\end{array}
$$

Problem (11)-(13) is equivalent to the minimization problem

$$
\min _{\varphi \in V_{r}\left(\Omega_{\varepsilon}\right)} J(\varphi)=\min _{\varphi \in V_{r}\left(\Omega_{\varepsilon}\right)}\left\{\frac{1}{r} \int_{\Omega_{\varepsilon}}|D(\varphi)|^{r} d x-\int_{\Omega_{\varepsilon}} \mathbf{f} \cdot \varphi d x\right\} .
$$

For $1<r<+\infty, J$ is strictly convex, proper, continuous and coercive, which give the existence and the uniqueness of the minimizer $\mathbf{v}^{\varepsilon} \in V_{r}\left(\Omega_{\varepsilon}\right)$ (see [34]). The pressure field is recovered using the De Rham or Tartar's constructions (see [80]).

\subsection{The filtration laws via two-scale asymptotic expansions: the power-law}

The conclusion of the previous subsection is that we can take dimensionless problem (11)-(13) as the starting point for the asymptotic analysis.

$\mathbf{v}^{\varepsilon}$ satisfies a priori estimate (9). Obtaining the a priori estimate for the pressure field $p^{\varepsilon}$ is more involved and we address the question in the next section. Motivated by the classical Darcy law, we expect that there would be a term $\mathbf{f}-\nabla_{x} p$ in the filtration law. Hence we expect to have the pressure $p^{\varepsilon}$ uniformly bounded in $L^{r^{\prime}}\left(\Omega_{\varepsilon}\right)$.

After having obtained the a priori estimates, we can proceed with formal twoscale asymptotic expansions. We will use the two-scale asymptotic expansion (1) to perform the formal homogenization of the system (11)-(13). Introducing the fast variable $y=x / \varepsilon$, we assume that the solution of (11)-(13) can be developed in the following way

$$
\left\{\begin{array}{l}
\mathbf{v}^{\varepsilon}(x)=\varepsilon^{r /(r-1)}\left\{\mathbf{v}^{0}(x, y)+\varepsilon \mathbf{v}^{1}(x, y)+\ldots\right\} \\
p^{\varepsilon}(x)=p^{0}(x, y)+\varepsilon p^{1}(x, y)+\ldots
\end{array}\right.
$$

The above two-scale expansion can be considered as a special case of the general expansions of this type from the monographs by Sanchez-Palencia [72] and Hornung 
[39]. The differential operators transform as follows

$$
\begin{gathered}
\nabla \cdot=\frac{1}{\varepsilon} \nabla_{y} \cdot+\nabla_{x} ; \quad D=\frac{1}{\varepsilon} D_{y}+D_{x} ; \\
\nabla=\frac{1}{\varepsilon} \nabla_{y}+\nabla_{x} ; \quad\left|D\left(\mathbf{v}^{\varepsilon}\right)\right|^{r-2}=\varepsilon^{(r-2) /(r-1)}\left|D_{y}\left(\mathbf{v}^{0}\right)+O(\varepsilon)\right|^{r-2} .
\end{gathered}
$$

Insertion of the two-scale asymptotic expansion into the incompressibility condition (12) yields

$$
\frac{1}{\varepsilon} \nabla_{y} \cdot \mathbf{v}^{0}+\nabla_{x} \cdot \mathbf{v}^{0}+\nabla_{y} \cdot \mathbf{v}^{1}=O(\varepsilon)
$$

Hence at order $O(1 / \varepsilon)$ we have

$$
\nabla_{y} \cdot \mathbf{v}^{0}=0 \quad \text { in } \quad Y_{F} ; \quad \mathbf{v}^{0}=0 \quad \text { on } \quad S
$$

and at order $O(1)$

$$
\nabla_{x} \cdot \mathbf{v}^{0}+\nabla_{y} \cdot \mathbf{v}^{1}=0 \quad \text { in } \quad Y_{F} ; \quad \mathbf{v}^{1}=0 \quad \text { on } \quad S .
$$

Integration of equation (16) over $Y_{F}$ yields the macroscopic mass conservation equation

$$
\nabla_{x} \cdot\left(\int_{Y_{F}} \mathbf{v}^{0}(x, y) d y\right)=0 \text { in } \Omega .
$$

Insertion of the two-scale expansion into the momentum equation (11) yields

$$
\begin{gathered}
-\left\{\frac{1}{\varepsilon} \nabla_{y} \cdot+\nabla_{x} \cdot\right\}\left\{\varepsilon\left|D_{y}\left(\mathbf{v}^{0}\right)+O(\varepsilon)\right|^{r-2}\left(D_{y}\left(\mathbf{v}^{0}\right)+O(\varepsilon)\right)\right\}+ \\
\frac{1}{\varepsilon} \nabla_{y} p^{0}+\nabla_{x} p^{0}+\nabla_{y} p^{1}+O(\varepsilon)=\mathbf{f} .
\end{gathered}
$$

At order $O(1 / \varepsilon)$, equation (18) yields

$$
\nabla_{y} p^{0}=0 \quad \Rightarrow p^{0}=p^{0}(x) .
$$

At order $O(1)$ equation (18) yields

$$
\nabla_{y} p^{1}-\nabla_{y} \cdot\left\{\left|D_{y}\left(\mathbf{v}^{0}\right)\right|^{r-2} D_{y}\left(\mathbf{v}^{0}\right)\right\}=\mathbf{f}(x)-\nabla_{x} p^{0}(x) \quad \text { in } \quad Y_{F} .
$$

Now we are able to write the resulting homogenized two-scale system. 


$$
\begin{gathered}
\nabla_{y} p^{1}-\nabla_{y} \cdot\left\{\left|D_{y}\left(\mathbf{v}^{0}\right)\right|^{r-2} D_{y}\left(\mathbf{v}^{0}\right)\right\}=\mathbf{f}(x)-\nabla_{x} p^{0}(x) \text { in } Y_{F} \times \Omega, \\
\nabla_{y} \cdot \mathbf{v}^{0}=0 \quad \text { in } Y_{F} \times \Omega ; \quad \mathbf{v}^{0}=0 \text { on } S \times \Omega, \\
\left\{\mathbf{v}^{0}, p^{1}\right\} \text { are } Y \text {-periodic, } \\
\nabla_{x} \cdot\left(\int_{Y_{F}} \mathbf{v}^{0}(x, y) d y\right)=0 \text { in } \Omega, \\
\mathbf{n} \cdot \int_{Y_{F}} \mathbf{v}^{0}(x, y) d y=0 \text { on } \partial \Omega .
\end{gathered}
$$

System (21)-(25) is called the two-pressures quasi-Newtonian Stokes problem.

Let

$$
V=\left\{\psi \in L^{r}\left(\Omega ; W_{p e r}^{1, r}\left(Y_{F}\right)^{3}\right) \mid \psi \text { satisfies conditions }(22)-(25)\right\} .
$$

Then the variational form of system (21)-(25) is

Find $\mathbf{v}^{0} \in V$ such that

$$
\int_{\Omega} \int_{Y_{F}}\left|D_{y}\left(\mathbf{v}^{0}\right)\right|^{r-2} D_{y}\left(\mathbf{v}^{0}\right): D_{y}(\psi) d y d x=\int_{\Omega} \int_{Y_{F}} \mathbf{f} \cdot \psi d y d x, \quad \forall \psi \in V .
$$

After [34], the strict monotonicity, continuity and coercivity of the operator yields existence of a unique solution for problem (26).

Similar to the Newtonian case,

$$
-\nabla_{y} \cdot\left\{\left|D_{y}\left(\mathbf{v}^{0}\right)\right|^{r-2} D_{y}\left(\mathbf{v}^{0}\right)\right\}-\mathbf{f}=0 \quad \text { in } \quad V^{\prime}
$$

means that $-\nabla_{y} \cdot\left\{\left|D_{y}\left(\mathbf{v}^{0}\right)\right|^{r-2} D_{y}\left(\mathbf{v}^{0}\right)\right\}-\mathbf{f}$ is an element of the subspace

$$
\tilde{V}=\left\{\nabla_{x} \varphi+\nabla_{y} \psi, \varphi \in W^{1, r /(r-1)}(\Omega) \text { and } \psi \in L^{r /(r-1)}\left(\Omega, L_{p e r}^{r /(r-1)}\left(Y_{F}\right) / \mathbb{R}\right)\right\}
$$

of $L^{r /(r-1)}\left(\Omega, W^{-1, r /(r-1)}\left(Y_{F}\right)^{3}\right)$.

The uniqueness of the pressure field is linked to the following surjectivity property:

Lemma 2. (see [5]) For any function $\theta \in L^{q}(\Omega)^{3}, 1<q<+\infty$, there exists $\sigma \in$ $L^{q}\left(\Omega ; W_{\text {per }}^{1, q}\left(Y_{F}\right)^{3}\right)$ such that

$$
\nabla_{y} \cdot \sigma=0 \quad \text { in } Y_{F} ; \quad \sigma=0 \quad \text { on } S ; \int_{Y_{F}} \sigma d y=\theta(x)
$$

and $\|\sigma\|_{L^{q}\left(\Omega ; W_{p e r}^{1, q}\left(Y_{F}\right)^{3}\right)} \leq C\|\theta\|_{L^{q}(\Omega)^{3}}$.

In the uniqueness proof, we get for the pressures differences $p=p_{1}^{0}-p_{2}^{0}$ and $\pi=$ $p_{1}^{1}-p_{2}^{1}, \nabla_{x} p+\nabla_{y} \pi=0$. Consequently, 


$$
\begin{gathered}
-\nabla_{x} p \cdot \int_{Y_{F}} \sigma(x, y) d y=0 \quad \forall \sigma \in W_{\text {per }}^{1, r}\left(Y_{F}\right)^{3}, \\
\nabla_{y} \cdot \sigma=0 \text { in } Y_{F} \times \Omega, \sigma=0 \text { on } S \times \Omega .
\end{gathered}
$$

Now using the above result we get $\nabla_{x} p=0$ which implies $p=0$ in $L_{0}^{r /(r-1)}(\Omega)$. Finally, $\nabla_{y} \pi=0$ and $\pi=0$.

Remark 2. After the discussion in [74] and [75], the filtration laws of the form

$$
U_{\text {filt }}=M\left(\dot{\gamma}_{\text {eff }}, K, \varphi\right) \nabla p
$$

are usually used in applications. $U_{\text {filt }}$ is the filtration velocity and $M$ is the effective mobility, defined as the ratio of the permeability to the effective viscosity and depending on the effective shear rate $\dot{\gamma}_{e f f}$, permeability $K$ and porosity $\varphi$. After our two-scale expansions, it makes sense to link the effective shear rate $\dot{\gamma}_{\text {eff }}$ to $\nabla_{x} p^{0}$.

The obtained two-scale filtration law (21)-(23) is not of Darcy law type and generally does not lead to the usual filtration law used in standard engineering treatment (e.g. as in [28], [81] and [68]):

$$
\mathbf{v}=\left(\frac{K}{\mu_{e f f}}\left[-\frac{\partial p}{\partial x}\right]\right)^{1 /(r-1)}
$$

The above filtration law is obtained by modelling a porous medium as a collection of long capillary tubes through which the fully developed laminar flow occurs.

If we suppose the flow only in the $x_{1}$ direction then the variables $x$ and $y$ in two pressures quasi-Newtonian Stokes system (21)-(25) can be separated. Then solving (21)-(25) leads to a non-linear one dimensional power-like law, identical to the one used in the engineering literature. In our notation it reads

$$
\int_{Y_{F}} \mathbf{v}(x, y) d y=\left|f-\frac{d p}{d x_{1}}\right|^{r^{\prime}-2} \cdot\left(f-\frac{d p}{d x_{1}}\right) \int_{Y_{F}} \mathbf{u}(y) d y,
$$

where $\mathbf{u}$ is the solution of two pressures quasi-Newtonian Stokes system (21)(25) for the right hand side of (21) being $\mathbf{e}^{1}=(1,0,0)$.

However, it should be noticed that this argument holds only in the one dimensional case. Our laws, except for a tubular porous medium, are nonlocal and they cannot be reduced to a multi-dimensional variant of (28), connecting the Darcy velocity $\mathbf{v}$ and some power of $\nabla p$. Most laboratory experiments are performed for onedimensional flows, which makes difficult observing any dimensional effect. From the engineering point of view, it is important to have not only a good laboratory prediction, but also the filtration laws for the oil fields.

In the case of tube flows, a detailed study of the filtration laws is in [21] and [55].

Remark 3. For more details on the formal two-scale expansions presented above we refer to [73] and [66]. 
Remark 4. The impossibility to separate slow scale $x$ and the fast scale $y$ in the homogenized momentum equation (21)-(23) has consequences to the numerical simulations. A numerical method of a good performance was introduced in [37]. A study of the analytic properties of the homogenized law was undertaken in [23].

\subsection{The filtration laws via two-scale asymptotic expansions: Carreau law}

The analogous procedure applies to the case of the Carreau-Yasuda law.

We start with the a priori estimate pour Carreau law. As before, we use Poincaré's inequality in a porous medium (7). In the Carreau-Yosuda law $\eta_{\infty} \approx 0$ and we neglect it. Furthermore, we suppose $\lambda=\lambda_{0} / \varepsilon$ and $1<r<2$. Then, after testing equation (4) with the solution we obtain

$$
\|\mathbf{v}\|_{L^{r}}+\varepsilon\|D(\mathbf{v})\|_{L^{r}} \leq C \varepsilon^{2}
$$

in the case of Carreau's law.

Hence the characteristic velocity $U$ is of order $O\left(\varepsilon^{2}\right)$ and the Reynolds number $\operatorname{Re}=\rho L_{0} U / \eta_{0}$ is small. Therefore, it is enough to consider the quasi-Newtonian Stokes equations.

Consequently, in the case of Carreau law (3), with $\eta_{\infty}=0$, we consider the dimensionless system

$$
\begin{gathered}
-\nabla \cdot\left\{\left(1+\frac{\lambda_{0}^{2}}{\varepsilon^{2}}\left|D\left(\mathbf{v}^{\varepsilon}\right)\right|^{2}\right)^{r / 2-1} D\left(\mathbf{v}^{\varepsilon}\right)\right\}+\nabla p^{\varepsilon}=\mathbf{f} \quad \text { in } \Omega_{\varepsilon} \\
\nabla \cdot \mathbf{v}^{\varepsilon}=0 \quad \text { in } \Omega_{\varepsilon} \\
\mathbf{v}^{\varepsilon}=0 \quad \text { on } \partial \Omega_{\varepsilon}
\end{gathered}
$$

Problem (30)-(32) is equivalent to the minimization problem

$$
\min _{\varphi \in V_{r}\left(\Omega_{\varepsilon}\right)} J(\varphi)=\min _{\varphi \in V_{r}\left(\Omega_{\varepsilon}\right)}\left\{\frac{\varepsilon^{2}}{r \lambda_{0}^{2}} \int_{\Omega_{\varepsilon}}\left(1+\frac{\lambda_{0}^{2}}{\varepsilon^{2}}\left|D\left(\mathbf{v}^{\varepsilon}\right)\right|^{2}\right)^{r / 2} d x-\int_{\Omega_{\varepsilon}} \mathbf{f} \cdot \varphi d x\right\} .
$$

For $1<r<+\infty, J$ is strictly convex, proper, continuous and coercive, which yields the existence and the uniqueness of the minimizer $\mathbf{v}^{\varepsilon} \in V_{r}\left(\Omega_{\varepsilon}\right)$. The pressure field is (again) recovered using the De Rham or Tartar's constructions.

$\mathbf{v}^{\varepsilon}$ satisfies a priori estimate (29) and we make a hypothesis that $p^{\varepsilon}$ is uniformly bounded in $L^{r^{\prime}}\left(\Omega_{\varepsilon}\right)$.

We will use the two-scale asymptotic expansion (1) to perform the formal homogenization of the system (30)-(32). Introducing the fast variable $y=x / \varepsilon$, we assume that the solution of (30)-(32) can be developed in the following way 


$$
\left\{\begin{array}{l}
\mathbf{v}^{\varepsilon}(x)=\varepsilon^{2}\left\{\mathbf{v}^{0}(x, y)+\varepsilon \mathbf{v}^{1}(x, y)+\ldots\right\} \\
p^{\varepsilon}(x)=p^{0}(x, y)+\varepsilon p^{1}(x, y)+\ldots
\end{array}\right.
$$

As before, the differential operators transform as follows

$$
\begin{gathered}
\nabla \cdot=\frac{1}{\varepsilon} \nabla_{y} \cdot+\nabla_{x} ; \quad D=\frac{1}{\varepsilon} D_{y}+D_{x} ; \quad \nabla=\frac{1}{\varepsilon} \nabla_{y}+\nabla_{x} ; \\
\left(1+\frac{\lambda_{0}^{2}}{\varepsilon^{2}}\left|D\left(\mathbf{v}^{\varepsilon}\right)\right|^{2}\right)^{r / 2-1}=\left|1+\lambda_{0}^{2}\right| D_{y}\left(\mathbf{v}^{0}\right)+\left.\left.O(\varepsilon)\right|^{2}\right|^{r / 2-1} .
\end{gathered}
$$

In the case of Carreau's law, the formal asymptotic expansion for the mass conservation equation is identical to the case of the power law:

Insertion of the 2-scale expansion into the incompressibility condition (31) yields

$$
\frac{1}{\varepsilon} \nabla_{y} \cdot \mathbf{v}^{0}+\nabla_{x} \cdot \mathbf{v}^{0}+\nabla_{y} \cdot \mathbf{v}^{1}=O(\varepsilon) .
$$

Hence at order $O(1 / \varepsilon)$ we have

$$
\nabla_{y} \cdot \mathbf{v}^{0}=0 \quad \text { in } \quad Y_{F} ; \quad \mathbf{v}^{0}=0 \quad \text { on } \quad S
$$

and at order $O(1)$

$$
\nabla_{x} \cdot \mathbf{v}^{0}+\nabla_{y} \cdot \mathbf{v}^{1}=0 \quad \text { in } \quad Y_{F} ; \quad \mathbf{v}^{1}=0 \quad \text { on } S .
$$

Integration of equation (35) over $Y_{F}$ yields the macroscopic mass conservation equation

$$
\nabla_{x} \cdot\left(\int_{Y_{F}} \mathbf{v}^{0}(x, y) d y\right)=0 \text { in } \Omega .
$$

Insertion of the two-scale expansion into the momentum equation (30) is slightly different and yields

$$
\begin{gathered}
-\left\{\frac{1}{\varepsilon} \nabla_{y} \cdot+\nabla_{x} \cdot\right\}\left\{\varepsilon\left|1+\lambda_{0}^{2}\right| D_{y}\left(\mathbf{v}^{0}\right)+\left.\left.O(\varepsilon)\right|^{2}\right|^{r / 2-1}\left(D_{y}\left(\mathbf{v}^{0}\right)+O(\varepsilon)\right)\right\} \\
+\frac{1}{\varepsilon} \nabla_{y} p^{0}+\nabla_{x} p^{0}+\nabla_{y} p^{1}+O(\varepsilon)=\mathbf{f} .
\end{gathered}
$$

At order $O(1 / \varepsilon)$, equation (37) yields

$$
\nabla_{y} p^{0}=0 \quad \Rightarrow p^{0}=p^{0}(x) .
$$

At order $O(1)$ equation (37) yields

$$
\nabla_{y} p^{1}-\nabla_{y} \cdot\left\{\left(1+\lambda_{0}^{2}\left|D_{y}\left(\mathbf{v}^{0}\right)\right|^{2}\right)^{r / 2-1} D_{y}\left(\mathbf{v}^{0}\right)\right\}=\mathbf{f}(x)-\nabla_{x} p^{0}(x) \quad \text { in } \quad Y_{F} .
$$

Now we are able to write the resulting homogenized two-scale system. 


$$
\begin{gathered}
\nabla_{y} p^{1}-\nabla_{y} \cdot\left\{\left(1+\lambda_{0}^{2}\left|D_{y}\left(\mathbf{v}^{0}\right)\right|^{2}\right)^{r / 2-1} D_{y}\left(\mathbf{v}^{0}\right)\right\}=\mathbf{f}(x)-\nabla_{x} p^{0}(x) \\
\text { in } Y_{F} \times \Omega, \\
\nabla_{y} \cdot \mathbf{v}^{0}=0 \quad \text { in } Y_{F} \times \Omega ; \quad \mathbf{v}^{0}=0 \text { on } S \times \Omega, \\
\quad\left\{\mathbf{v}^{0}, p^{1}\right\} \text { are } Y-\text { periodic } \\
\nabla_{x} \cdot\left(\int_{Y_{F}} \mathbf{v}^{0}(x, y) d y\right)=0 \text { in } \Omega, \\
\mathbf{n} \cdot \int_{Y_{F}} \mathbf{v}^{0}(x, y) d y=0 \text { on } \partial \Omega .
\end{gathered}
$$

System (40)-(44) is called the two-pressures Carreau-Stokes problem.

Let

$$
V=\left\{\psi \in L^{r}\left(\Omega ; W_{p e r}^{1, r}\left(Y_{F}\right)^{3}\right) \mid \psi \text { satisfies conditions }(41)-(44)\right\} .
$$

Then the variational form of system (40)-(44) is

Find $\mathbf{v}^{0} \in V$ such that

$$
\begin{gathered}
\int_{\Omega} \int_{Y_{F}}\left(1+\lambda_{0}^{2}\left|D_{y}\left(\mathbf{v}^{0}\right)\right|^{2}\right)^{r / 2-1} D_{y}\left(\mathbf{v}^{0}\right): D_{y}(\psi) d y d x= \\
\int_{\Omega} \int_{Y_{F}} \mathbf{f} \cdot \psi d y d x, \quad \forall \psi \in V .
\end{gathered}
$$

After [34], the strict monotonicity, continuity and coercivity of the operator yields existence of a unique solution for problem (26).

Similar to the Newtonian case,

$$
-\nabla_{y} \cdot\left\{\left(1+\lambda_{0}^{2}\left|D_{y}\left(\mathbf{v}^{0}\right)\right|^{2}\right)^{r / 2-1} D_{y}\left(\mathbf{v}^{0}\right)\right\}-\mathbf{f}=0 \quad \text { in } \quad V^{\prime}
$$

means that $-\nabla_{y} \cdot\left\{\left|D_{y}\left(\mathbf{v}^{0}\right)\right|^{r-2} D_{y}\left(\mathbf{v}^{0}\right)\right\}-\mathbf{f}$ is an element of the subspace

$$
\tilde{V}=\left\{\nabla_{x} \varphi+\nabla_{y} \psi, \varphi \in W^{1, r /(r-1)} \text { and } \psi \in L^{r /(r-1)}\left(\Omega, L_{p e r}^{r /(r-1)}\left(Y_{F}\right) / \mathbb{R}\right)\right\}
$$

of $L^{r /(r-1)}\left(\Omega, W^{-1, r /(r-1)}\left(Y_{F}\right)^{3}\right)$.

The uniqueness of the pressure field is proved similarly like to the power law case.

Remark 5. Finding the filtration laws of the form

$$
U_{\text {filt }}=M\left(\dot{\gamma}_{e f f}, K, \varphi\right) \nabla p
$$

is even more complicated in this case. The consideration from [23] apply to the Carreau law case as well. 


\subsection{The filtration laws via two-scale asymptotic expansions: Bingham fluid case}

In this subsection we discuss briefly the filtration laws for a Bingham fluid (a viscoplastic fluid) in a porous medium.

As in subsection 2.3 let $\mathbf{v}$ be the velocity, $p$ the pressure and $D(\mathbf{v})=(\nabla \mathbf{v}+$ $\left.\nabla \mathbf{v}^{t}\right) / 2$ the rate-of-strain tensor. In the case of the Bingham fluid, the stress tensor $\sigma$ is given by

$$
\sigma=-p I+\left(2 \eta_{0}+\frac{g}{\dot{\gamma}}\right) D(\mathbf{v})
$$

where $\eta_{0}$ is the viscosity and $\dot{\gamma}=\sqrt{|D(\mathbf{v})|^{2} / 2}$ is the shear rate. The deviatoric stress tensor $\tau$, i.e. the part of the total stress tensor that is zero at equilibrium, is then a nonlinear function of the shear rate $D(\mathbf{v})$,

$$
\tau=\left(2 \eta_{0}+\frac{g}{\dot{\gamma}}\right) D(\mathbf{v})
$$

Constitutive law (46) is valid only if $\dot{\gamma} \neq 0$.

In [33], Duvaut and Lions have shown that this constitutive law is equivalent with the following one:

$$
\left\{\begin{array}{l}
|\tau|^{2} / 2 \leq g \Rightarrow D(\mathbf{v})=0 \\
|\tau|^{2} / 2>g \Rightarrow D(\mathbf{v})=(1-g / \dot{\gamma}) \tau /\left(2 \eta_{0}\right)
\end{array}\right.
$$

This is a threshold law: as long as the shear stress is below $g$, the fluid behaves as a rigid solid. When the value of the shear stress exceeds $g$, the fluid flows and obeys a nonlinear constitutive law. Moreover, the fluid is incompressible.

We will deal with the variational formulation of the problem. Let

$$
V\left(\Omega_{p}\right)=\left\{\psi \mid \psi \in H_{0}^{1}\left(\Omega_{p}\right)^{3}, \nabla \cdot \psi=0 \text { in } \Omega_{p}\right\} .
$$

Then the variational problem reads as follows:

Find $\mathbf{v} \in V(\Omega)$ such that

$$
\begin{gathered}
2 \eta_{0} \int_{\Omega_{p}} D(\mathbf{v}): D(\psi-\mathbf{v}) d x+2 g \int_{\Omega_{p}}(\dot{\gamma}(\psi)-\dot{\gamma}(\mathbf{v})) d x \geq \\
\int_{\Omega_{p}} \mathbf{f} \cdot(\psi-\mathbf{v}) d x, \forall \psi \in V\left(\Omega_{p}\right) .
\end{gathered}
$$

Problem (48) was studied in [33] and existence and uniqueness were proved. Furthermore, the corresponding differential interpretation result was established:

Proposition 2. Let $\mathbf{v}$ be the solution for (48). Then there exist a matrix $\mathscr{M}(x)$ and a function $p \in L_{0}^{2}\left(\Omega_{p}\right)$ such that 


$$
\begin{gathered}
\mathscr{M}_{i j} \in L^{\infty}\left(\Omega_{p}\right) ; \mathscr{M}_{i j}=\mathscr{M}_{j i}, \forall i, j ; \operatorname{Tr}(\mathscr{M})=0 \\
|\mathscr{M}| \leq 1 \text { a.e. on } \Omega_{p} ; \mathscr{M}: D(\mathbf{v})=|D(\mathbf{v})| \text { a.e. on } \Omega_{p} \\
\quad-\eta_{0} \Delta \mathbf{v}-g \sqrt{2} \nabla \cdot \mathscr{M}=\mathbf{f}-\nabla p .
\end{gathered}
$$

Following [45] we will study the following dimensionless Bingham flow model in a porous medium

Find $\mathbf{v}^{\varepsilon} \in V\left(\Omega_{\varepsilon}\right)$ such that

$$
\begin{gathered}
2 \eta_{0} \varepsilon^{2} \int_{\Omega_{\varepsilon}} D\left(\mathbf{v}^{\varepsilon}\right): D\left(\psi-\mathbf{v}^{\varepsilon}\right) d x+2 g \varepsilon \int_{\Omega_{\varepsilon}}\left(\dot{\gamma}(\psi)-\dot{\gamma}\left(\mathbf{v}^{\varepsilon}\right)\right) d x \geq \\
\int_{\Omega_{\varepsilon}} \mathbf{f} \cdot\left(\psi-\mathbf{v}^{\varepsilon}\right) d x, \forall \psi \in V\left(\Omega_{\varepsilon}\right) .
\end{gathered}
$$

and study the behavior of the problem in the limit $\varepsilon \rightarrow 0$. The corresponding twoscale asymptotic expansion was developed in [45] and a detailed study of the homogenized problem undertaken. The computations are involved and the interested reader can consult the article [45].

We will see that for a variational inequality (49) direct use of the two-scale convergence is rigorous and shorter than using formal asymptotic expansions.

\section{An introduction to the 2-scale convergence with special attention to the two-scale lower semi-continuity}

We start with recalling basic facts from functional analysis. Let $Y=(0,1)^{n}$ be the unit cube in $\mathbb{R}^{n}$ and $Q$ a bounded open set in $\mathbb{R}^{n}$.

Definition 1. A sequence $\left\{u^{\varepsilon}\right\}$ in $L^{q}(Q), 1 \leq q<+\infty$ is said to converge weakly to $u \in L^{q}(Q)$ if

$$
\lim _{\varepsilon \rightarrow 0} \int_{Q} u^{\varepsilon}(x) v(x) d x=\int_{Q} u(x) v(x) d x, \quad \forall v \in L^{q *}(Q) ; q^{*}=q /(q-1) .
$$

The notation is $u^{\varepsilon} \rightarrow u$. If $q=+\infty$, then $q^{*}=1$ and we have the weak ${ }^{*}$ convergence in $L^{\infty}(Q)$.

After [24], the weak topology on a Banach space $E$ is the coarsest topology in which the linear forms are continuous.

Lemma 3. (a property of the mean value - see e.g. [40]) Let $f$ be a Y-periodic function. Then

$$
\begin{gathered}
f\left(\frac{x}{\varepsilon}\right) \rightarrow \frac{1}{|Y|} \int_{Y} f(y) \text { dy weakly in } L^{q}(Q) \quad \text { for } 1 \leq q<+\infty \\
\text { and weak }{ }^{*} \text { in } L^{\infty}(Q), \text { as } \varepsilon \rightarrow 0
\end{gathered}
$$


Example 1. $\sin \frac{x}{\varepsilon} \rightarrow 0$ weakly in $L_{\text {loc }}^{q}\left(\mathbb{R}^{n}\right)$ for $1 \leq q<+\infty$ and weak* in $L^{\infty}(Q)$, as $\varepsilon \rightarrow 0$.

Our difficulty is that in the homogenization problems we will have to calculate limits of the type

$$
\lim _{\varepsilon \rightarrow 0} \int_{Q} A\left(\frac{x}{\varepsilon}\right) \nabla u^{\varepsilon} \psi d x
$$

with weakly converging $\nabla u^{\varepsilon}$ and $A\left(\frac{x}{\varepsilon}\right)$. Their product will not converge in general to the product of the weak limits.

Example 2. $\sin \frac{x}{\varepsilon} \rightarrow 0$ weakly, as $\varepsilon \rightarrow 0$, but $\sin ^{2} \frac{x}{\varepsilon} \rightarrow 1 / 2$ weakly and not to 0 .

Therefore the weak convergence is not well adapted to our needs and the strong convergence is out of reach for problems with oscillations.

There are several methods to pass to the limit in such products, like Tartar's energy ethod and the compensated compactness (see e.g. [40]).

Here we will present the two-scale convergence method, which involves a convergence which is weaker than the strong convergence but stronger than the weak convergence. We will see that it captures successfully the oscillations. It was introduced by Nguetseng in [63] and developed by Allaire in [5].

Definition 2. The bounded sequence $\left\{w^{\varepsilon}\right\} \subset L^{q}(Q), 1<q<+\infty$, is said to two-scale converge to a limit $w \in L^{q}(Q \times Y)$ if and only if for any $\sigma \in L^{q /(q-1)}\left(Q ; \overline{\left.C_{\text {per }}^{\infty}(Y)\right)}\right.$ ("per" denotes $Y$-periodicity) one has

$$
\lim _{\varepsilon \rightarrow 0} \int_{Q} w^{\varepsilon}(x) \sigma\left(x, \frac{x}{\varepsilon}\right) d x=\int_{Q} \int_{Y} w(x, y) \sigma(x, y) d y d x .
$$

We note that for $\sigma$ the values on the diagonal $y=x / \varepsilon$ have to make sense and for $\sigma \in L^{2}(Q \times Y)$ it is not the case.

(Admissible functions) A function $f$ belongs to $L^{q}\left(Q, C_{p e r}(Y)\right), 1<q<+\infty$ if and only if there exists a subset $E$ of measure zero in $Q$ such that

a For any $x \in Q \backslash E$, the function $y \rightarrow f(x, y)$ is continuous and $Y$ - periodic.

b For any $y \in Y$, the function $x \rightarrow f(x, y)$ is measurable.

c The function $x \rightarrow \sup _{y \in Y}|f(x, y)|$ has finite $L^{q}(Q)$ norm.

Furthermore, the function $x \rightarrow f\left(x, \frac{x}{\varepsilon}\right)$ is measurable and

$$
\begin{aligned}
\left\|f\left(x, \frac{x}{\varepsilon}\right)\right\|_{L^{2}(Q)} & \leq\|f\|_{L^{2}\left(Q ; C_{p e r}(Y)\right)} ; \\
\lim _{\varepsilon \rightarrow 0} \int_{Q}\left|f\left(x, \frac{x}{\varepsilon}\right)\right|^{2} d x & =\int_{Q} \int_{Y}|f(x, y)|^{2} d x d y .
\end{aligned}
$$

The spaces $L^{q}\left(Q, C_{\text {per }}(Y)\right), L_{\text {loc }}^{q}(Y, C(\bar{Q}))$ and $C\left(\bar{Q}, C_{\text {per }}(Y)\right), 1 \leq q<+\infty$, are all separable Banach spaces, dense in $L^{q}(Q \times Y)$. We refer to [49] for more details about the notion of the two-scale convergence in $L^{q}$-setting and the admissibility conditions for the test functions. 
Example 3. $\sin \frac{x}{\varepsilon} \rightarrow 0$ weakly but $\sin \frac{x}{\varepsilon} \rightarrow \sin y$ in two-scales, thus retaining the information on the shape of oscillations present in the sequence. Note that the twoscale convergence will not see the oscillations which are not in resonance with those of test functions: $u\left(x, x / \varepsilon^{2}\right) \rightarrow \int_{Y} u(x, y) d y$ in two-scales, as $\varepsilon \rightarrow 0$.

The basic Compactness Theorem for the two-scale convergence: With the weak convergence/topology, we have less open sets than when using the strong topology but more compact sets. The situation is similar with the two-scale convergence. Boundedness of a sequence will be sufficient for relative two-scale compactness. The proof is based on

The sequential Banach-Alaoglu theorem (see [24]): Let $X$ be a separable Banach space. Then every bounded sequence in $X^{*}$ has a weak* convergent subsequence.

Theorem 1. Let $\left\{u^{\varepsilon}\right\}$ be a bounded sequence in $L^{q}(Q), 1<q<+\infty$. Then there exists a subsequence, denoted by the same subscripts, and $u^{0} \in L^{q}(Q \times Y)$ such that $\left\{u^{\varepsilon}\right\}$ two-scales converges to $u^{0}$.

Proof. Step 1. Let $\psi \in L^{q /(q-1)}\left(Q, C_{\text {per }}(Y)\right)$. We define a sequence of functionals $\left\{\mu_{\varepsilon}\right\}$ on $L^{q /(q-1)}\left(Q, C_{p e r}(Y)\right)$ by

$$
<\mu_{\varepsilon}, \psi>=\int_{Q} u^{\varepsilon}(x) \psi\left(x, \frac{x}{\varepsilon}\right) d x .
$$

It is easy to see that

$$
\begin{aligned}
\left|<\mu_{\varepsilon}, \psi>\right| \leq & \left|\int_{Q} u^{\varepsilon}(x) \psi\left(x, \frac{x}{\varepsilon}\right) d x\right| \leq C|| \psi\left(x, \frac{x}{\varepsilon}\right) \|_{L^{q /(q-1)}(Q)} \\
& \leq C\|\psi(x, y)\|_{L^{q /(q-1)}\left(Q, C_{p e r}(Y)\right)}
\end{aligned}
$$

Hence the sequence $\left\{\mu_{\varepsilon}\right\}$ is bounded in $\left(L^{q /(q-1)}\left(Q, C_{p e r}(Y)\right)\right)^{*}$.

Step 2. $L^{q /(q-1)}\left(Q, C_{p e r}(Y)\right)$ is separable and by the sequential Banach-Alaoglu theorem, there is a subsequence of $\left\{\mu_{\varepsilon}\right\}$ and a $\mu \in\left(L^{q /(q-1)}\left(Q, C_{p e r}(Y)\right)\right)^{*}$ such that $\mu_{\varepsilon} \rightarrow \mu$ in the weak ${ }^{*}$ topology in $\left(L^{q /(q-1)}\left(Q, C_{p e r}(Y)\right)\right)^{*}$. Thus

$$
<\mu_{\varepsilon}, \psi>\rightarrow<\mu, \psi>, \quad \forall \psi \in L^{q /(q-1)}\left(Q, C_{p e r}(Y)\right) .
$$

Step 3. Obviously

$$
|<\mu, \psi>| \leq C\left(\int_{Q}\left|\psi\left(x, \frac{x}{\varepsilon}\right)\right|^{q /(q-1)} d x\right)^{1-1 / q} \leq\left. C|| \psi(x, y)\right|_{L^{q /(q-1)}(Q \times Y)} .
$$

Since $L^{q /(q-1)}\left(Q, C_{\text {per }}(Y)\right)$ is dense in $L^{q /(q-1)}(Q \times Y)$, we can extend $\mu$ to a bounded linear functional on $L^{q /(q-1)}(Q \times Y)$. The extension is denoted $\tilde{\mu}$.

$\tilde{\mu}$ satisfies estimate (53) and by the Riesz representation theorem $\tilde{\mu}$ can be identified with an element $u^{0} \in L^{q}(Q \times Y)$. Then we have 


$$
\begin{gathered}
\lim _{\varepsilon \rightarrow 0} \int_{Q} u^{\varepsilon}(x) \psi\left(x, \frac{x}{\varepsilon}\right) d x=\lim _{\varepsilon \rightarrow 0}\left\langle\mu_{\varepsilon}, \psi>=<\mu, \psi>\right. \\
=\int_{Q} \int_{Y} u^{0}(x, y) \sigma(x, y) d y d x
\end{gathered}
$$

for every $\psi \in L^{q /(q-1)}\left(Q, C_{p e r}(Y)\right)$. This completes the proof.

It is well-known that for PDEs the weak compactness in Sobolev spaces is of importance. It is the same with the two-scale compactness. We follow the approach of Allaire from [5]. Applying the basic compactness theorem for the two-scale convergence first to the functions and then to their derivatives, and then simply comparing the limits yields

Proposition 3. (see [5])

(a) Let $w^{\varepsilon}$ and $\varepsilon \nabla w^{\varepsilon}$ be bounded sequences in $L^{q}(Q), 1<q<+\infty$. Then there exists a function $w \in L^{q}\left(Q ; W_{p e r}^{1, q}(Y)\right)$ and a subsequence such that both $w^{\varepsilon}$ and $\varepsilon \nabla w^{\varepsilon}$ two-scale converge to $w$ and $\nabla_{y} w$, resp.

(b) Let $w^{\varepsilon}$ and $\nabla w^{\varepsilon}$ be bounded sequences in $L^{q}(Q), 1<q<+\infty$. Then there exists functions $w \in W^{1, q}(Q)$ and $w_{1} \in L^{q}\left(Q ; W_{p e r}^{1, q}(Y)\right)$ and a subsequence such that both $w^{\varepsilon}$ and $\nabla w^{\varepsilon}$ two-scale converge to $w$ and $\nabla_{x} w+\nabla_{y} w_{1}$, resp.

(c) Let $\sigma \in L_{\text {per }}^{q}(Y)$, define $\sigma^{\varepsilon}(x)=\sigma\left(\frac{x}{\varepsilon}\right)$, and let the sequence $\left\{w^{\varepsilon}\right\} \subset L^{q}(Q)$ two-scale converges to a limit $w \in L^{q}(Q \times Y)$. Then $\left\{\sigma^{\varepsilon} w^{\varepsilon}\right\}$ two-scale converges to a limit $\sigma w$.

(d) Let $\mathbf{v}^{\varepsilon}$ be a divergence-free bounded sequence in $L^{q}(Q)^{n}, 1<q<+\infty$, which two-scale convergences to $\mathbf{v}^{0} \in L^{q}(Q \times Y)^{n}$. then, the two-scale limit satisfies $\operatorname{div}_{y} \mathbf{v}^{0}(x, y)=0$ a.e. in $Q \times Y$ and $\int_{Y} \operatorname{div}_{x} \mathbf{v}^{0}(x, y) d y=0$.

Remark 6. Strong Convergence $\Rightarrow$ Two-scale Convergence $\Rightarrow$ Weak convergence

Weak Convergence $\nRightarrow$ Two-scale Convergence $\nRightarrow$ Strong convergence

After recalling these basic properties we give a sequential lower semicontinuity result for two-scale convergence in $L^{q}, 1<q<+\infty$.

Proposition 4. (see [5]) Let $\Phi: \mathbb{R}^{n} \rightarrow \mathbb{R}^{+}$be a continuous function satisfying $0 \leq$ $\Phi(\lambda)$ for all $\lambda \in \mathbb{R}^{n}, \sigma \in C_{0}^{\infty}\left(Q ; C_{p e r}^{\infty}(Y)\right)^{n}$, and $\sigma^{\varepsilon}(x)=\sigma\left(x, \frac{x}{\varepsilon}\right)$. Then

$$
\lim _{\varepsilon \rightarrow 0} \int_{Q} \Phi\left(\sigma^{\varepsilon}\right) d x=\int_{Q} \int_{Y} \Phi(\sigma) d y d x
$$

Furthermore, let $\Phi$ in addition be strictly convex and $C^{1}$ in $\mathbb{R}^{n^{2}}$, satisfying

$$
c|\lambda|^{q} \leq \Phi(\lambda) \leq C\left(1+|\lambda|^{q}\right), \quad \forall \lambda \in \mathbb{R}^{n}, 1<q<+\infty .
$$

Then, if $\mathbf{v}^{\varepsilon}$ is a bounded sequence from $L^{q}(\Omega)^{n}$ which two-scale converges towards $\mathbf{v}$, we have

$$
\liminf _{\varepsilon \rightarrow 0} \int_{Q} \Phi\left(\mathbf{v}^{\varepsilon}\right) d x \geq \int_{Q} \int_{Y} \Phi(\mathbf{v}) d y d x
$$


Remark 7. In fact the two scale semi-continuity result is not directly stated in [5], but it is contained in the proof of Theorem 3.3, pages 1500-1503. For $q=2$ the result is stated in [7] as theorem 3.7 on page 243. For the confort of the reader we recall the argument from [5]:

Since $\Phi$ is convex and $C^{1}$, we have

$$
\Phi\left(\mathbf{v}^{\varepsilon}\right) \geq \Phi\left(\psi\left(x, \frac{x}{\varepsilon}\right)\right)+\nabla_{v} \Phi\left(\psi\left(x, \frac{x}{\varepsilon}\right)\right)\left(\mathbf{v}^{\varepsilon}-\psi\left(x, \frac{x}{\varepsilon}\right)\right),
$$

for every $\psi \in C_{0}^{\infty}\left(\Omega ; C_{p e r}^{\infty}(Y)\right)^{n}$, implying

$$
\begin{gathered}
\liminf _{\varepsilon \rightarrow 0} \int_{Q} \Phi\left(\mathbf{v}^{\varepsilon}\right) d x \geq \liminf _{\varepsilon \rightarrow 0} \int_{Q} \Phi\left(\psi\left(x, \frac{x}{\varepsilon}\right) d x+\right. \\
\liminf _{\varepsilon \rightarrow 0} \int_{Q} \nabla_{\nu} \Phi\left(\psi\left(x, \frac{x}{\varepsilon}\right)\right)\left(\mathbf{v}^{\varepsilon}-\psi\left(x, \frac{x}{\varepsilon}\right)\right) d x=\int_{Q} \int_{Y} \Phi(\psi(x, y)) d y d x+ \\
\int_{Q} \int_{Y} \nabla_{\nu} \Phi(\psi(x, y))(\mathbf{v}(x, y)-\psi(x, y)) d x d y .
\end{gathered}
$$

Next, we take for $\psi$ a sequence of smooth functions $\psi_{k} \in C_{0}^{\infty}\left(\Omega ; C_{p e r}^{\infty}(Y)\right)^{n}$ which converges to $\mathbf{v}$ strongly in $L^{q}(Q \times Y)^{n}$. Due to the growth conditions on $\Phi$ and smoothness, inequality (56) holds also in the limit $\psi_{k} \rightarrow \mathbf{v}$ in the two-scale sense and we obtain the inequality (55). Note that the coercivity is not required for the lower semi-continuity.

In several applications (Bingham flows, friction, ... ) the functional

$$
\psi \rightarrow \int_{Q}|\psi(x)| d x
$$

arises. We have

Proposition 5. Let $\left\{\mathbf{v}^{\varepsilon}\right\}$ be a bounded sequence from $\left(L^{q}(\Omega)\right)^{n}, 1<q<+\infty$, which two-scale converges towards $\mathbf{v}$, we have

$$
\liminf _{\varepsilon \rightarrow 0} \int_{Q}\left|\mathbf{v}^{\varepsilon}(x)\right| d x \geq \int_{Q} \int_{Y}|\mathbf{v}(x, y)| d y d x .
$$

Proof. The functions $f_{\delta}=\sqrt{|x|^{2}+\delta^{2}}-\delta$ are $C^{1}$ with partial derivatives $\frac{\partial f_{\delta}}{\partial x_{j}}=$ $x_{j} / \sqrt{|x|^{2}+\delta^{2}}, j=1, \ldots, n$. We have

$$
\int_{Q}|| \mathbf{v}^{\varepsilon}\left|-f_{\delta}\left(\mathbf{v}^{\varepsilon}\right)\right| d x \leq c \delta
$$

and

$$
\sqrt{\left|\mathbf{v}^{\varepsilon}\right|^{2}+\delta^{2}}-\delta \geq \sqrt{|\psi|^{2}\left(x, \frac{x}{\varepsilon}\right)+\delta^{2}}-\delta+\sum_{j=1}^{n} \frac{\psi_{j}\left(x, \frac{x}{\varepsilon}\right)}{\sqrt{|\psi|^{2}\left(x, \frac{x}{\varepsilon}\right)+\delta^{2}}}\left(v_{j}^{\varepsilon}-\psi_{j}\left(x, \frac{x}{\varepsilon}\right)\right)
$$


for every smooth $\psi(x, y)$. Hence we have

$$
\begin{gathered}
\liminf _{\varepsilon \rightarrow 0} \int_{Q}\left(\sqrt{\left|\mathbf{v}^{\varepsilon}\right|^{2}+\delta^{2}}-\delta\right) d x \geq \int_{Q} \int_{Y}\left(\sqrt{|\psi|^{2}(x, y)+\delta^{2}}-\delta\right) d x d y+ \\
\sum_{j=1}^{n} \int_{Q} \int_{Y} \frac{\psi_{j}(x, y)}{\sqrt{|\psi|^{2}(x, y)+\delta^{2}}}\left(v_{j}-\psi_{j}(x, y)\right) d x d y
\end{gathered}
$$

Now we take a sequence of smooth functions $\psi$, periodic in $y$, which strongly converges to $\mathbf{v}$. It yields

$$
\begin{gathered}
\liminf _{\varepsilon \rightarrow 0} \int_{Q}\left(\sqrt{\left|\mathbf{v}^{\varepsilon}\right|^{2}+\delta^{2}}-\delta\right) d x \geq \int_{Q} \int_{Y}\left(\sqrt{|\mathbf{v}|^{2}(x, y)+\delta^{2}}-\delta\right) d x d y \Rightarrow \\
\liminf _{\varepsilon \rightarrow 0} \int_{Q}\left|\mathbf{v}^{\varepsilon}(x)\right| d x \geq \liminf _{\varepsilon \rightarrow 0} \int_{Q}\left(\sqrt{\left|\mathbf{v}^{\varepsilon}\right|^{2}+\delta^{2}}-\delta\right) d x-C \delta \geq \\
\int_{Q} \int_{Y}\left(\sqrt{|\mathbf{v}|^{2}(x, y)+\delta^{2}}-\delta\right) d x d y-C \delta \geq \int_{Q} \int_{Y}|\mathbf{v}| d x-C \delta, \quad \forall \delta>0
\end{gathered}
$$

and the proposition is proved. $\square$

Remark 8. It is important to note that two-scale convergence is a tool adapted to the particular problem one wants to solve. Consequently, other two-scale convergences can be introduced. An example are the problems with chemical reactions/biological processes on surfaces $\Gamma^{\varepsilon}$. Then the appropriate tool is the two-scale convergence on the surfaces developed in [62], [6] and [50]. Another example is the two-scale convergence with drift, designed to handle homogenization of reaction-diffusion equations with large Péclet and Damkohler's numbers. For details we refer to [54], [8] and [9].

\section{The a priori estimates for the pressure and the two-scale limits in the case of the power law viscosity}

In order to use the two-scale convergence, we first need a priori estimates.

We recall the estimate (9), valid in the case of the power-law viscosity:

$$
\left\|\mathbf{v}^{\varepsilon}\right\|_{L^{r}}+\varepsilon\left\|D\left(\mathbf{v}^{\varepsilon}\right)\right\|_{L^{r}} \leq C \varepsilon^{r /(r-1)} .
$$

In order to investigate the behavior of solutions to (11)-(13), as $\varepsilon \rightarrow 0$, we need to extend $\mathbf{v}^{\varepsilon}$ and $p^{\varepsilon}$ to the whole of $\Omega$. We extend $\mathbf{v}^{\varepsilon}$ by zero in $\Omega \backslash \Omega_{\varepsilon}$. It is well known that extension by zero preserves $L^{q}$ and $W_{0}^{1, q}$ norms for $1<q<\infty$.

Extending the pressure is a much more difficult task. The extension is closely related to the construction of the restriction operator $R_{q} \in \mathscr{L}\left(W^{1, q}(Y)^{n}, W_{S}^{1, q}\left(Y_{F}\right)^{n}\right)$, $n=2,3$, where $W_{S}^{1, q}\left(Y_{F}\right)=\left\{\mathbf{z} \in W^{1, q}\left(Y_{F}\right): z=0\right.$ on $\left.S\right\}$. 
A priori estimates for the pressure are derived using the a priori estimates for the velocity and the equation:

$$
\begin{gathered}
\nabla p^{\varepsilon}=\mathbf{f}+\nabla \cdot\left\{\left|D\left(\mathbf{v}^{\varepsilon}\right)\right|^{r-2} D\left(\mathbf{v}^{\varepsilon}\right)\right\} \Rightarrow \\
<\nabla p^{\varepsilon}, \psi>=\int_{\Omega_{\varepsilon}}\left(\left|D\left(\mathbf{v}^{\varepsilon}\right)\right|^{r-2} D\left(\mathbf{v}^{\varepsilon}\right): D(\psi)+\mathbf{f} \cdot \psi\right) d x, \quad \forall \psi \in W_{0}^{1, r}\left(\Omega_{\varepsilon}\right)^{3}
\end{gathered}
$$

Hence the pressure $p^{\varepsilon}$ satisfies the inequality

$$
\left\|\nabla p^{\varepsilon}\right\|_{W^{-1, r^{\prime}}\left(\Omega_{\varepsilon}\right)^{3}} \leq C \varepsilon .
$$

The functional space $W^{-1, r^{\prime}}\left(\Omega_{\varepsilon}\right)^{3}$ changes with $\varepsilon$ and estimate (59) is difficult to use. Our strategy is to extend the pressure to the solid part of the porous medium.

Following the idea of Lipton, Avellaneda [46] and using the constructions by Tartar and Allaire (see [4], [7] and the Appendix of [72]) we define the extension of pressure $p^{\varepsilon}$ by

$$
\tilde{p}^{\varepsilon}= \begin{cases}p^{\varepsilon}, & \text { in } \Omega_{\varepsilon} \\ \frac{1}{\left|\varepsilon\left(Y_{F}+i\right)\right|} \int_{Y_{F_{i}}^{\varepsilon}} p^{\varepsilon}, & \text { in the } Y_{S_{i}}^{\varepsilon} \text { for each } i\end{cases}
$$

where $Y_{F_{i}}^{\varepsilon}$ is the fluid part of the cell $Y_{i}^{\varepsilon}$. Note that solid part of the porous medium is an union of all $Y_{S_{i}}^{\varepsilon}$. We have

Proposition 6. (see [7] ) The pressure extension $\tilde{p}^{\varepsilon} \in L_{0}^{r^{\prime}}(\Omega)$ of the function $p^{\varepsilon}$, defined by (60) satisfies the estimate

$$
\left\|\tilde{p}^{\varepsilon}\right\|_{L^{r^{\prime}}(\Omega)}+\left\|\nabla \tilde{p}^{\varepsilon}\right\|_{W^{-1, r^{\prime}}(\Omega)^{n}} \leq C, n=2,3 .
$$

Furthermore for arbitrary sequence $\left\{w^{\varepsilon}\right\} \subset L_{0}^{r}(\Omega)^{n}$ which converges weakly to 0 , we have

$$
\int_{\Omega} \tilde{p}^{\varepsilon} w^{\varepsilon} \rightarrow 0 \quad \text { as } \varepsilon \rightarrow 0
$$

Proposition 7. Let $\left\{\mathbf{v}^{\varepsilon}, p^{\varepsilon}\right\}$ be corresponding solutions of the power-law system (11)-(13). Then there exist subsequences of $\left\{\mathbf{v}^{\varepsilon}\right\}$ and $\left\{\tilde{p}^{\varepsilon}\right\}$ (again denoted by the same symbols) and functions $\mathbf{v}_{0}^{*} \in L^{r}(\Omega \times Y)^{3}, p^{*} \in L_{0}^{r /(r-1)}(\Omega)$ and $\nabla_{y} \mathbf{v}_{0}^{*} \in L^{r}(\Omega \times$ $Y)^{9}$ such that

$$
\begin{gathered}
\varepsilon^{-r /(r-1)} \mathbf{v}^{\varepsilon} \rightarrow \mathbf{v}_{0}^{*} \quad \text { in the two-scale sense in } L^{r}, \\
\varepsilon^{-1 /(r-1)} \nabla \mathbf{v}^{\varepsilon} \rightarrow \nabla_{y} \mathbf{v}_{0}^{*} \in L^{r}(\Omega \times Y)^{9} \text { in the two-scale sense in } L^{r}, \\
\varepsilon^{-r /(r-1)} \mathbf{v}^{\varepsilon} \rightarrow \mathbf{v}^{*}=\int_{Y_{F}} \mathbf{v}_{0}^{*} d y \text { weakly in } L^{r}(\Omega)^{3}, \\
\tilde{p}^{\varepsilon} \rightarrow p^{*} \text { in } L_{0}^{r /(r-1)}(\Omega),
\end{gathered}
$$


as $\varepsilon \rightarrow 0$.

Proof. Proof of Proposition 7 follows directly from (9) and (61), through the compactness results stated in Proposition 3. The pressure convergence (66) follows the formal two-scale expansion:

Let $\psi \in C_{0}^{\infty}\left(\Omega ; C_{\text {per }}^{\infty}\left(Y_{F}\right)\right)^{3}$ such that $\psi(x, y)=0$ on $S$ for (a.e.) $x \in \Omega$ and set $\psi^{\varepsilon}(x)=\psi\left(x, \frac{x}{\varepsilon}\right)$. We test equation (21) with $\varepsilon \psi^{\varepsilon}$. It yields

$$
0=\lim _{\varepsilon \rightarrow 0} \int_{\Omega} \tilde{p}^{\varepsilon} \nabla_{y} \cdot \psi^{\varepsilon} d x=\int_{\Omega} \int_{Y} p^{*} \nabla_{y} \cdot \psi(x, y) d x d y=<\nabla_{y} p^{*}, \psi>_{\Omega \times Y} .
$$

Hence $p^{*}$ is independent of $y$. The information is enough for passing to the limit in the terms containing the pressure, but after [72], (62) implies also the strong convergence of $\tilde{p}^{\varepsilon}$.

Using the incompressibility and the weak convergence (65), we find out that the average $\mathbf{v}^{*}$ satisfies the equations

$$
\nabla_{x} \cdot \mathbf{v}^{*}=0 \text { in } \Omega, \quad \mathbf{v}^{*} \cdot \mathbf{n}=0 \text { on } \partial \Omega .
$$

Lemma 4. $\mathbf{v}_{0}^{*} \in L^{r}\left(\Omega ; W_{S}^{1, r}\left(Y_{F}\right)^{3}\right)$ and $\nabla_{y} \cdot \mathbf{v}_{0}^{*}=0$ in $Y_{F}$.

Proof. Let $\psi$ be a smooth function. Then

$$
\begin{aligned}
0 & =-\int_{\Omega_{\varepsilon}} \varepsilon^{-r /(r-1)} \mathbf{v}^{\varepsilon} \cdot \varepsilon \nabla \psi\left(x, \frac{x}{\varepsilon}\right) d x \rightarrow-\int_{\Omega} \int_{Y_{F}} \mathbf{v}_{0}^{*} \cdot \nabla_{y} \psi d y d x=0 . \\
\Rightarrow \nabla_{y} \cdot \mathbf{v}_{0}^{*} & =0 \text { in } Y_{F} \cdot \square
\end{aligned}
$$

Proposition 8. The functions $\mathbf{v}_{0}^{*}$ and $p^{*}$ defined, respectively, by (63) and (66) satisfy the two-pressures quasi-Newtonian Stokes problem (21)-(25).

Proof. It remains only to justify the momentum equation (21):

$$
\nabla \cdot\left\{\left|D_{y}\left(\mathbf{v}_{0}^{*}\right)\right|^{r-2} D_{y}\left(\mathbf{v}_{0}^{*}\right)\right\}+\nabla_{y} \pi(x, y)=\mathbf{f}-\nabla_{x} p^{*}(x) \text { in } Y_{F} \times \Omega .
$$

We use equation

$$
\int_{\Omega_{\varepsilon}}\left|D\left(\mathbf{v}^{\varepsilon}\right)\right|^{r-2} D\left(\mathbf{v}^{\varepsilon}\right): D(\psi) d x+<\nabla p^{\varepsilon}-\mathbf{f}, \psi>=0, \forall \psi \in W_{0}^{1, r}\left(\Omega_{\varepsilon}\right) .
$$


Using Minty's lemma ${ }^{2}$ we write it in as a minimization problem with a given pressure:

$$
\begin{gathered}
\int_{\Omega} \frac{1}{r}|\varepsilon D(\psi)|^{r} d x-\int_{\Omega} \frac{1}{r}\left|\varepsilon D\left(\varepsilon^{-r /(r-1)} \mathbf{v}^{\varepsilon}\right)\right|^{r} d x \geq \\
-\left\langle f-\nabla \tilde{p}^{\varepsilon}, \psi-\varepsilon^{-r /(r-1)} \mathbf{v}^{\varepsilon}\right\rangle_{\Omega}, \quad \forall \psi \in W_{0}^{1, r}\left(\Omega_{\varepsilon}\right)^{3} .
\end{gathered}
$$

Next we choose $\psi \in C_{0}^{\infty}\left(\Omega ; C_{\text {per }}^{\infty}\left(Y_{F}\right)\right)^{3}$ such that $\psi(x, y)=0$ on $S$ for (a.e.) $x \in$ $\Omega, \nabla_{y} \cdot \psi=0$ in $Y_{F}$ and set $\psi^{\varepsilon}(x)=\psi\left(x, \frac{x}{\varepsilon}\right)$.

We insert $\psi=\psi^{\varepsilon}$ as a test function in (69). It yields

$$
-\left\langle\nabla \tilde{p}^{\varepsilon}, \psi^{\varepsilon}\right\rangle_{\Omega}=\int_{\Omega} \tilde{p}^{\varepsilon} \nabla_{x} \cdot \psi^{\varepsilon} \rightarrow \int_{\Omega} \int_{Y} p^{*} \nabla_{x} \cdot \psi(x, y) d x d y, \text { as } \varepsilon \rightarrow 0 .
$$

The above limit and Proposition 4 imply

$$
\begin{gathered}
\int_{\Omega} \int_{Y} \frac{1}{r}\left|D_{y}(\psi)\right|^{r} d x d y-\int_{\Omega} \int_{Y} \frac{1}{r}\left|D_{y}\left(\mathbf{v}_{0}^{*}\right)\right|^{r} d x d y \geq \\
\left\langle\mathbf{f}-\nabla p^{*}(x), \int_{Y}\left(\psi-\mathbf{v}_{0}^{*}\right) d y\right\rangle_{\Omega} .
\end{gathered}
$$

Using again Minty's lemma and de Rham's formula yield

$$
\begin{gathered}
-\nabla_{y} \cdot\left\{\left|D_{y}\left(\mathbf{v}_{0}^{*}\right)\right|^{r-2} D_{y}\left(\mathbf{v}_{0}^{*}\right)\right\}+\nabla_{y} \pi(x, y)=\mathbf{f}-\nabla p^{*}(x) \text { in } Y_{F} \\
\nabla_{y} \cdot \mathbf{v}_{0}^{*}=0 \text { in } Y_{F}, \quad \mathbf{v}_{0}^{*}=0 \text { on } S
\end{gathered}
$$

and (21) is justified.

Therefore we justified rigorously the two-pressures quasi-Newtonian Stokes problem. The uniqueness theorem from subsection 2.1 implies that the whole sequence converges towards $\left\{\mathbf{v}_{0}^{*}, p^{*}\right\}=\left\{\mathbf{v}^{0}, p^{0}\right\}$.

\subsection{A priori estimates and the two-scale convergence for the case of the law of Carreau}

We recall the Carreau-Stokes system, corresponding to Carreau law (3):

\footnotetext{
${ }^{2}$ Minty's lemma (see [34])) Let $F$ be a convex lower semi-continuous and proper functional on a reflexive Banach space $B$. Then for $u \in B$ the following three conditions are equivalent to each other:
a) $\quad u$ solves the problem $\inf _{v \in B} F(v)$.
b) $\quad\left\langle F^{\prime}(u), v-u>\geq 0, \quad \forall v \in B\right.$.

c) $\quad<F^{\prime}(v), v-u>\geq 0, \quad \forall v \in B$.
} 


$$
\begin{gathered}
-\nabla \cdot\left\{\left(1+\frac{\lambda_{0}^{2}}{\varepsilon^{2}}\left|D\left(\mathbf{v}^{\varepsilon}\right)\right|^{2}\right)^{r / 2-1} D\left(\mathbf{v}^{\varepsilon}\right)\right\}+\nabla p^{\varepsilon}=\mathbf{f} \text { in } \Omega_{\varepsilon} \\
\nabla \cdot \mathbf{v}^{\varepsilon}=0 \quad \text { in } \Omega_{\varepsilon} \\
\mathbf{v}^{\varepsilon}=0 \quad \text { on } \partial \Omega_{\varepsilon} .
\end{gathered}
$$

We also recall the a priori estimate (10) for the velocity:

$$
\left\|\mathbf{v}^{\varepsilon}\right\|_{L^{r}}+\varepsilon\left\|D\left(\mathbf{v}^{\varepsilon}\right)\right\|_{L^{r}} \leq C \varepsilon^{2} .
$$

In order to investigate the behavior of solutions to (71)-(73), as $\varepsilon \rightarrow 0$, we need to extend $v^{\varepsilon}$ and $p^{\varepsilon}$ to the whole of $\Omega$. We extend $v^{\varepsilon}$ by zero in $\Omega \backslash \Omega_{\varepsilon}$. It is well known that extension by zero preserves $L^{q}$ and $W_{0}^{1, q}$ norms for $1<q<\infty$.

Extending the pressure is a much more difficult task. A priori estimates for the pressure are derived using the a priori estimates for the velocity and the momentum equation (30):

$$
\begin{gathered}
\nabla p^{\varepsilon}=\mathbf{f}+\nabla \cdot\left\{\left(1+\frac{\lambda_{0}^{2}}{\varepsilon^{2}}\left|D\left(\mathbf{v}^{\varepsilon}\right)\right|^{2}\right)^{r / 2-1} D\left(\mathbf{v}^{\varepsilon}\right)\right\} \Rightarrow \\
<\nabla p^{\varepsilon}, \psi>=\int_{\Omega_{\varepsilon}}\left(\left(1+\frac{\lambda_{0}^{2}}{\varepsilon^{2}}\left|D\left(\mathbf{v}^{\varepsilon}\right)\right|^{2}\right)^{r / 2-1} D\left(\mathbf{v}^{\varepsilon}\right): D(\psi)+\mathbf{f} \cdot \psi\right) d x \\
\forall \psi \in W_{0}^{1, r}\left(\Omega_{\varepsilon}\right)^{3} .
\end{gathered}
$$

Hence the pressure $p^{\varepsilon}$ satisfies the inequality

$$
\left\|\nabla p^{\varepsilon}\right\|_{W^{-1, r^{\prime}}\left(\Omega_{\varepsilon}\right)^{3}} \leq C \varepsilon, \quad r^{\prime}=r /(r-1) .
$$

Extension of the pressure to the solid part of the porous medium is done again using formula (60) and estimate (61) is valid again..

Furthermore for arbitrary sequence $\left\{w^{\varepsilon}\right\} \subset L_{0}^{r}(\Omega)^{n}$ which converges weakly to 0 we have

$$
\int_{\Omega} \tilde{p}^{\varepsilon} w^{\varepsilon} \rightarrow 0 \quad \text { as } \varepsilon \rightarrow 0
$$

Proposition 9. Let $\left\{\mathbf{v}^{\varepsilon}, p^{\varepsilon}\right\}$ be the corresponding solutions of the Carreau-Stokes system (71)-(73). Then there exist subsequences of $\left\{\mathbf{v}^{\varepsilon}\right\}$ and $\left\{\tilde{p}^{\varepsilon}\right\}$ (again denoted by the same symbols) and functions $\mathbf{v}_{0}^{*} \in L^{r}(\Omega \times Y)^{3}, p^{*} \in L_{0}^{r^{\prime}}(\Omega)$ and $\nabla_{y} \mathbf{v}_{0}^{*} \in$ $L^{r}(\Omega \times Y)^{9}$ such that

$$
\begin{gathered}
\varepsilon^{-2} \mathbf{v}^{\varepsilon} \rightarrow \mathbf{v}_{0}^{C} \quad \text { in the two-scale sense in } L^{r}, \\
\varepsilon^{-1} \nabla \mathbf{v}^{\varepsilon} \rightarrow \nabla_{y} \mathbf{v}_{0}^{C} \in L^{r}(\Omega \times Y)^{9} \text { in the two-scale sense in } L^{r}, \\
\varepsilon^{-2} \mathbf{v}^{\varepsilon} \rightarrow \mathbf{v}^{C}=\int_{Y_{F}} \mathbf{v}_{0}^{C} d y \text { weakly in } L^{r}(\Omega)^{3}, \\
\tilde{p}^{\varepsilon} \rightarrow p^{C} \text { in } L_{0}^{r^{\prime}}(\Omega),
\end{gathered}
$$

as $\varepsilon \rightarrow 0$. 
Derivation of the macro and micro level mass conservation laws in the case of Carreau law is exactly the same as in the case of the power law. Only the momentum equations differs slightly.

Proposition 10. The functions $\mathbf{v}_{0}^{C}$ and $p^{C}$ defined, respectively, by (77) and (80) satisfy the two-pressures Carreau-Stokes problem (40)-(44).

Proof. It remains only to justify the momentum equation (40):

$$
\begin{gathered}
\nabla_{y} p^{1}-\nabla_{y} \cdot\left\{\left(1+\lambda_{0}^{2}\left|D_{y}\left(\mathbf{v}_{0}^{C}\right)\right|^{2}\right)^{r / 2-1} D_{y}\left(\mathbf{v}_{0}^{C}\right)\right\}=\mathbf{f}(x)-\nabla_{x} p^{C}(x) \\
\text { in } Y_{F} \times \Omega .
\end{gathered}
$$

We use the variational equation

$$
\begin{gathered}
\int_{\Omega_{\varepsilon}}\left(1+\frac{\lambda_{0}^{2}}{\varepsilon^{2}}\left|D\left(\mathbf{v}^{\varepsilon}\right)\right|^{2}\right)^{r / 2-1} D\left(\mathbf{v}^{\varepsilon}\right): D(\psi) d x+<\nabla p^{\varepsilon}-\mathbf{f}, \psi>=0, \\
\forall \psi \in W_{0}^{1, r}\left(\Omega_{\varepsilon}\right)^{3} .
\end{gathered}
$$

and write it in as a minimization problem for a given pressure:

$$
\begin{gathered}
\int_{\Omega} \frac{1}{r \lambda_{0}^{2}}\left(1+\lambda_{0}^{2}|\varepsilon D(\psi)|^{2}\right)^{r / 2} d x-\int_{\Omega} \frac{1}{r \lambda_{0}^{2}}\left(1+\lambda_{0}^{2}\left|\varepsilon^{-1} D\left(\mathbf{v}^{\varepsilon}\right)\right|^{2}\right)^{r / 2} d x \geq \\
-\left\langle f-\nabla \tilde{p}^{\varepsilon}, \psi-\varepsilon^{-2} \mathbf{v}^{\varepsilon}\right\rangle_{\Omega}, \quad \forall \psi \in W_{0}^{1, r}\left(\Omega_{\varepsilon}\right)^{3} .
\end{gathered}
$$

Now we choose $\psi \in C_{0}^{\infty}\left(\Omega ; C_{\text {per }}^{\infty}\left(Y_{F}\right)\right)^{3}$ such that $\psi(x, y)=0$ on $S$ for (a.e.) $x \in$ $\Omega, \nabla_{y} \cdot \psi=0$ in $Y_{F}$ and define $\psi^{\varepsilon}(x)=\psi\left(x, \frac{x}{\varepsilon}\right)$.

We insert $\psi=\psi^{\varepsilon}$ in (82). Then

$$
-\left\langle\nabla \tilde{p}^{\varepsilon}, \psi^{\varepsilon}\right\rangle_{\Omega}=\int_{\Omega} \tilde{p}^{\varepsilon} \nabla_{x} \cdot \psi^{\varepsilon} \rightarrow \int_{\Omega} \int_{Y} p^{C} \nabla_{x} \cdot \psi(x, y) d x d y \text { as } \varepsilon \rightarrow 0 .
$$

The above limit and Proposition 4 imply

$$
\begin{gathered}
\int_{\Omega} \int_{Y} \frac{1}{r}\left|D_{y}(\psi)\right|^{r} d x d y-\int_{\Omega} \int_{Y} \frac{1}{r}\left|D_{y}\left(\mathbf{v}_{0}^{C}\right)\right|^{r} d x d y \geq \\
\left\langle\mathbf{f}-\nabla p^{C}(x), \int_{Y}\left(\psi-\mathbf{v}_{0}^{C}\right) d y\right\rangle_{\Omega} .
\end{gathered}
$$

After recalling Minty's lemma, using de Rham's formula yields

$$
\begin{gathered}
-\nabla_{y} \cdot\left\{\left(1+\lambda_{0}^{2}\left|D_{y}\left(\mathbf{v}_{0}^{C}\right)\right|^{2}\right)^{r / 2-1} D_{y}\left(\mathbf{v}_{0}^{C}\right)\right\}+\nabla_{y} \pi(x, y)=\mathbf{f}-\nabla p^{C}(x) \text { in } Y_{F} \\
\nabla_{y} \cdot \mathbf{v}_{0}^{C}=0 \text { in } Y_{F}, \quad \mathbf{v}_{0}^{C}=0 \text { on } S
\end{gathered}
$$

and (40) is justified. $\square$

Therefore we justified rigorously the two-pressures Carreau-Newtonian Stokes problem. The uniqueness theorem from subsection 2.1 implies that the whole sequence converges towards $\left\{\mathbf{v}_{0}^{C}, p^{C}\right\}=\left\{\mathbf{v}^{0}, p^{0}\right\}$. 
Remark 9. We note that other scalings are discussed in [22] . In other cases, depending on the scaling of $\lambda$, the limit could be either the classical Darcy law or the power law.

\subsection{A priori estimates and the two-scale convergence for the case of the Bingham flow}

In the case of the Bingham flow through a porous medium we study variational problem (49).

Find $\mathbf{u}_{\varepsilon} \in V\left(\Omega_{\varepsilon}\right)$ such that

$$
\begin{gathered}
2 \eta_{0} \varepsilon^{2} \int_{\Omega_{\varepsilon}} D\left(\mathbf{u}_{\varepsilon}\right): D\left(\psi-\mathbf{u}_{\varepsilon}\right) d x+2 g \varepsilon \int_{\Omega_{\varepsilon}}\left(\dot{\gamma}(\psi)-\dot{\gamma}\left(\mathbf{u}_{\varepsilon}\right)\right) d x \geq \\
\int_{\Omega_{\varepsilon}} \mathbf{f} \cdot\left(\psi-\mathbf{u}_{\varepsilon}\right) d x, \quad \forall \psi \in V\left(\Omega_{\varepsilon}\right) .
\end{gathered}
$$

and study the behavior of the solution $\mathbf{u}_{\varepsilon}$ to problem 84 in the limit $\varepsilon \rightarrow 0$.

We start with estimates for the velocity $\mathbf{u}_{\varepsilon}$, then we obtain a priori estimates for the pressure and extend the pressure to the solid part of the porous medium.

Proposition 11. Let $\left(\mathbf{u}_{\varepsilon}, p_{\varepsilon}\right)$ be a solution (49). Then we have

$$
\begin{gathered}
\left\|\mathbf{u}_{\varepsilon}\right\|_{L^{2}\left(\Omega_{\varepsilon}\right)^{3}} \leq C, \\
\varepsilon\left\|\nabla \mathbf{u}_{\varepsilon}\right\|_{L^{2}\left(\Omega_{\varepsilon}\right)^{9}} \leq C, \\
\left\|\nabla p_{\varepsilon}\right\|_{H^{-1}\left(\Omega_{\varepsilon}\right)^{3}} \leq C \varepsilon .
\end{gathered}
$$

Proof. Proof of the estimates (85) and (86) is obtained by taking the solution $\mathbf{u}_{\varepsilon}$ as a test function in (49). Next, from (49)) we get the inequality

$$
\left|<\nabla p_{\varepsilon}, v>_{\Omega_{\varepsilon}}\right| \leq\left|(f, v)_{\Omega_{\varepsilon}}\right|+\left|2 \eta_{0} \varepsilon^{2} \int_{\Omega_{\varepsilon}} D\left(\mathbf{u}_{\varepsilon}\right): D(\psi) d x\right|+g \sqrt{2} \varepsilon \int_{\Omega_{\varepsilon}}|D(v)| d x
$$

and (87) follows

We extend velocity $\mathbf{u}_{\varepsilon}$ by zero to the $\Omega \backslash \Omega_{\varepsilon}$ and denote the extension by the same symbol. Obviously estimates (85) and (86) remain valid and the extension is divergence free too. The extension of the pressure $p_{\varepsilon}$ is constructed as before and we summarize its properties in the following lemma:

Proposition 12. The pressure extension $\tilde{p}^{\varepsilon} \in L_{0}^{2}(\Omega)$ of the function $p_{\varepsilon}$, defined by (60) satisfies the estimate

$$
\left\|\tilde{p}_{\varepsilon}\right\|_{L^{2}(\Omega)}+\left\|\nabla \tilde{p}_{\varepsilon}\right\|_{H^{-1}(\Omega)^{n}} \leq C .
$$


Furthermore for arbitrary sequence $\left\{w^{\varepsilon}\right\} \subset L_{0}^{2}(\Omega)^{n}$ which converges weakly to 0 we have

$$
\int_{\Omega} \tilde{p}_{\varepsilon} w^{\varepsilon} \rightarrow 0 \quad \text { as } \varepsilon \rightarrow 0 .
$$

Proposition 13. Let $\left\{\mathbf{u}_{\varepsilon}, p_{\varepsilon}\right\}$ be the corresponding solutions of the Bingham system (49). Then there exist subsequences of $\left\{\mathbf{u}_{\varepsilon}\right\}$ and $\left\{\tilde{p}_{\varepsilon}\right\}$ (again denoted by the same symbols) and functions $\mathbf{u}_{0}^{B} \in L^{2}(\Omega \times Y)^{3}, p^{B} \in L_{0}^{2}(\Omega)$ and $\nabla_{y} \mathbf{u}_{0}^{B} \in L^{2}(\Omega \times Y)^{9}$ such that

$$
\begin{gathered}
\mathbf{u}_{\varepsilon} \rightarrow \mathbf{u}_{0}^{B} \quad \text { in the two-scale sense in } L^{2}, \\
\varepsilon \nabla \mathbf{u}_{\varepsilon} \rightarrow \nabla_{y} \mathbf{u}_{0}^{B} \in L^{r}(\Omega \times Y)^{9} \text { in the two-scale sense in } L^{2}, \\
\mathbf{u}_{\varepsilon} \rightarrow \mathbf{u}^{B}=\int_{Y_{F}} \mathbf{u}_{0}^{B} \text { dy weakly in } L^{2}(\Omega)^{3}, \\
\tilde{p}_{\varepsilon} \rightarrow p^{B} \text { in } L_{0}^{2}(\Omega),
\end{gathered}
$$

as $\varepsilon \rightarrow 0$.

Derivation of the macro and micro level mass conservation laws in the case of the Bingham flow is exactly the same as before. Only passing to the limit in the momentum equation is different.

Proposition 14. Let

$$
\begin{gathered}
V\left(Y_{F}\right)=\left\{\psi \mid \psi \in H_{p e r}^{1}\left(Y_{F}\right)^{3}, \psi=0 \text { on } S, \nabla_{y} \cdot \psi=0 \text { in } Y_{F}\right\}, \\
\mathscr{W}=\left\{\phi \mid \phi \in L^{2}\left(\Omega ; V\left(Y_{F}\right)\right), \nabla_{x} \cdot \int_{Y_{F}} \phi d y=0 \text { in } \Omega\right. \\
\text { and } \left.\mathbf{n} \cdot \int_{Y_{F}} \phi d v=0 \text { on } \partial \Omega\right\} .
\end{gathered}
$$

The functions $\mathbf{u}_{0}^{B} \in \mathscr{W}$ and $p^{B}$ defined, respectively, by (91) and (94) satisfy the following two-pressures Bingham variational inequality

$$
\begin{gathered}
2 \eta_{0} \int_{Y_{F}} D_{y}\left(\mathbf{u}_{0}^{B}\right): D\left(\psi-\mathbf{u}_{0}^{B}\right) d y+2 g \int_{Y_{F}}\left(\dot{\gamma}_{y}(\psi)-\dot{\gamma}_{y}\left(\mathbf{u}_{0}^{B}\right)\right) d x \geq \\
\int_{Y_{F}}\left(\mathbf{f}-\nabla_{x} p^{B}\right) \cdot\left(\psi-\mathbf{u}_{0}^{B}\right) d x, \quad \forall \psi \in V\left(Y_{F}\right) .
\end{gathered}
$$

Proof. We choose $\psi \in C_{0}^{\infty}\left(\Omega ; C_{\text {per }}^{\infty}\left(Y_{F}\right)\right)^{3}$ such that $\psi(x, y)=0$ on $S$ for (a.e.) $x \in$ $\Omega, \nabla_{y} \cdot \psi=0$ in $Y_{F}$ and define $\psi^{\varepsilon}(x)=\psi\left(x, \frac{x}{\varepsilon}\right)$.

Then we write (49) in the form

$$
\begin{gathered}
2 \eta_{0} \varepsilon^{2} \int_{\Omega_{\varepsilon}} D\left(\mathbf{u}_{\varepsilon}\right): D\left(\psi^{\varepsilon}\right) d x+\int_{\Omega_{\varepsilon}}\left(g \sqrt{2} \varepsilon\left|D\left(\psi^{\varepsilon}\right)\right|-p_{\varepsilon} \nabla_{x} \cdot \psi^{\varepsilon}-\mathbf{f} \cdot \psi^{\varepsilon}\right) d x \geq \\
\int_{\Omega_{\varepsilon}}\left(2 \eta_{0} \varepsilon^{2}\left|D\left(\mathbf{u}_{\varepsilon}\right)\right|^{2}+g \sqrt{2} \varepsilon\left|D\left(\mathbf{u}_{\varepsilon}\right)\right|-\mathbf{f} \cdot \mathbf{u}_{\varepsilon}\right) d x
\end{gathered}
$$


Next as $\varepsilon \rightarrow 0$ we get

$$
\begin{gathered}
\int_{\Omega} \tilde{p}^{\varepsilon} \nabla_{x} \cdot \psi^{\varepsilon} \rightarrow \int_{\Omega} \int_{Y} p^{B}(x) \nabla_{x} \cdot \psi(x, y) d x d y \\
\int_{\Omega_{\varepsilon}} g \varepsilon\left|D\left(\psi^{\varepsilon}\right)\right| d x \rightarrow \int_{\Omega} \int_{Y_{F}} g\left|D_{y}(\psi)\right| d y .
\end{gathered}
$$

Next

$$
\begin{gathered}
2 \eta_{0} \varepsilon^{2} \int_{\Omega_{\varepsilon}} D\left(\mathbf{u}_{\varepsilon}\right): D\left(\psi^{\varepsilon}\right) d x \rightarrow \int_{\Omega} \int_{Y_{F}} 2 \eta_{0} D_{y}\left(\mathbf{u}_{0}^{B}\right): D_{y}(\psi) d y d x \\
\int_{\Omega_{\varepsilon}}\left(2 \eta_{0} \varepsilon^{2}\left|D\left(\mathbf{u}_{\varepsilon}\right)\right|^{2}+g \sqrt{2} \varepsilon\left|D\left(\mathbf{u}_{\varepsilon}\right)\right|\right) d x \geq \\
\int_{\Omega} \int_{Y_{F}}\left(2 \eta_{0}\left|D_{y}\left(\mathbf{u}_{0}^{B}\right)\right|^{2}+g \sqrt{2}\left|D_{y}\left(\mathbf{u}_{0}^{B}\right)\right|\right) d y d x \text { as } \varepsilon \rightarrow 0 .
\end{gathered}
$$

Hence we passed to the limit in all terms and the Proposition is proved.

Therefore we justified rigorously the two-pressures Bingham-Stokes problem (95), (41)-(44). The uniqueness theorem from [45] implies that the whole sequence converges towards $\left\{\mathbf{u}_{0}^{B}, p^{B}\right\}$.

\subsection{Concluding remarks on filtration laws for non-Newtonian fluids}

(a) Solving cell problems in the case of the quasi-Newtonian and Bingham flows poses numerical difficulties. See [37] for an efficient numerical method and [23] for an analytic study of the filtration laws, corresponding to the power and Carreau law viscosities.

(b) For the (formal) homogenization of a linear Oldroyd fluid in a bundle of capillary tubes at low Reynolds and Deborah numbers see [43]. Very little is known concerning filtration laws for non-Newtonian fluids, which are more complicated than the quasi-Newtonian ones discussed in this chapter.

(c) Homogenization in Orlicz spaces of the quasi-Newtonian flow equations with more general viscosity laws was undertaken in [41]. Some viscosity laws, as e.g. Ellis' law

$$
\eta_{r}(\dot{\gamma})=\frac{\eta_{0}}{1+\left(\dot{\gamma} \eta_{r}(\dot{\gamma})\right)^{\alpha-1} / \tau_{1 / 2}^{\alpha-1}}
$$

enter into the implicit constitutive laws considered in [25].

(d) An interesting open question is to get a corrector result of the type $\varepsilon^{-r /(r-1)} \mathbf{v}^{\varepsilon}$ $-\mathbf{v}^{0}(x, x / \varepsilon) \rightarrow 0$ in $L^{r}(\Omega)^{3}$, as $\varepsilon \rightarrow 0$. For the Newtonian case we refer to [7]. 


\section{Homogenization of the linearized ionic transport equations in rigid periodic porous media}

The quasi-static transport of an electrolyte through an electrically charged porous medium is an important and well-known multiscale problem in geosciences and porous materials modeling. An $N$-component electrolyte is a dilute solution of $N$ species of charged particles, or ions, in a fluid which saturates a charged porous medium. The porous medium can be either rigid or deformable.

The overall behavior of such a system is controlled by several phenomena. First there is an effective filtration. It is caused by the hydrodynamic flow in the pore space, heavily influenced by the charge distributions of the system. Second, there is a migration of ions because of an electric field. Third, the diffusive transport of the ions takes place. Finally, we have to take into account electrokinetic phenomena due to the electric double layer (EDL) which is formed as a result of the interaction of the electrolyte solution neutralizing the charge of the solid phase at the pore solidliquid interface.

The EDL can be split into several parts, depending on the strength of the electrostatic coupling. There is a condensed layer of heavily adsorbed ions depending on the molecular nature of the interface. It is generally known as the Stern layer and its characteristic width (the Gouy length) is typically less than one nanometer. Adjacent to the Stern layer the electrostatic diffuse layer or Debye's layer is formed, where the ion density varies. The EDL is the union of Stern and diffuse layers. The thickness of the diffuse layer is predicted by the Debye length $\lambda_{D}$ which depends on the electrolyte concentration. For low to moderate electrolyte concentrations $\lambda_{D}$ is in the nanometric range. Outside Debye's layer, in the remaining bulk fluid, the solvent can be considered as electrically neutral.

A detailed, mathematically oriented, presentation of the fundamental concepts of electroosmotic flow in nanochannels can be found in the book [42] by Karniadakis et al., pages 447-470, from which we borrow the notations and definitions.

In the case of porous media with large pores, the electro-osmotic effects are modeled by introducing an effective slip velocity (the Smoluchowski slip) at the solidliquid interfaces. Such models are not valid for numerous systems, such as clays because the characteristic pore size is also of the order of the EDL size (a few hundreds of nanometers or even less). Therefore the Debye's layer fills largely the pores and its effect cannot anymore be modeled by an effective slip boundary condition at the liquid-solid interface.

In this section, we consider continuum physics equations as the right model for the description of porous media at the pore scale where the EDL phenomena and the pore geometry interact and will search to upscale them. It would allow to derive and validate the macroscopic models used for engineering simulations (see the works of Adler and collaborators [2], [3], [15], [30], [38], [51], [71]). The typical length scale for which the continuum mechanics equations are valid is confirmed to be both experimentally (see e.g. [27]) and theoretically [52, 32] close to 1 nanometer. Therefore, at the microscopic level we couple the incompressible Stokes equations 
for the fluid with the electrokinetic model made of a global electrostatic equation and one convection-diffusion equation for each type of ions of an $\mathrm{N}$-component electrolyte in a dilute Newtonian solvent.

We start with the following mass conservation laws

$$
\operatorname{div}\left(\mathbf{j}_{i}+\mathbf{v} n_{i}\right)=0 \quad \text { in } \quad \Omega_{p}, \quad i=1, \ldots, N
$$

where $\Omega_{p}$ is the pore space of the porous medium, $i$ denotes the solute species, $\mathbf{v}$ is the hydrodynamic velocity and $n_{i}$ is the $i$ th species concentration. For each species $i, \mathbf{v} n_{i}$ is its convective flux and $\mathbf{j}_{i}$ its migration-diffusion flux.

The solute velocity satisfies the incompressible Stokes equations with a forcing term consisting of an exterior hydrodynamical force $\mathbf{f}$ and of the electric force

$$
\begin{aligned}
\eta \Delta \mathbf{v}=\mathbf{f}+\nabla p+e \sum_{j=1}^{N} z_{j} n_{j} \nabla \Psi \quad \text { in } \quad \Omega_{p}, \\
\operatorname{div} \mathbf{v}=0 \quad \text { in } \quad \Omega_{p} \quad \text { and } \quad \mathbf{v}=0 \quad \text { on } \quad \partial \Omega_{p} \backslash \partial \Omega,
\end{aligned}
$$

where $\eta>0$ is the shear viscosity, $\mathbf{f}$ is the external body force, $p$ is the pressure, $e$ is the elementary charge, $z_{i}$ is the charge number of the species $i$ and $\Psi$ is the electrostatic potential.

We assume that all valencies $z_{j}$ are different integers. If not, we lump together different ions with the same valency. We rank them by increasing order and we assume that they are both anions and cations, namely positive and negative valencies,

$$
z_{1}<z_{2}<\ldots<z_{N}, \quad z_{1}<0<z_{N},
$$

and we denote by $j^{+}$and $j^{-}$the sets of positive and negative valencies.

The migration-diffusion flux $\mathbf{j}_{i}$ is given by the following semi-linear relationship

$$
\mathbf{j}_{i}=-\sum_{j=1}^{N} L_{i j}\left(n_{1}, \ldots, n_{N}\right)\left(\nabla \mu_{j}+z_{j} e \nabla \Psi\right), \quad i=1, \ldots, N
$$

where $L_{i j}\left(n_{1}, \ldots, n_{N}\right)$ is the Onsager coefficient between $i$ and $j$ and $\mu_{j}$ is the chemical potential of the species $j$ given by

$$
\mu_{j}=\mu_{j}^{0}+k_{B} T \ln n_{j}+k_{B} T \ln \gamma_{j}\left(n_{1}, \ldots, n_{N}\right), \quad j=1, \ldots, N
$$

with $\gamma_{j}$ being the activity coefficient of the species $j, k_{B}$ is the Boltzmann constant, $\mu_{j}^{0}$ is the standard chemical potential expressed at infinite dilution and $T$ is the absolute temperature. The Onsager tensor $\left[L_{i j}\right]$ consists of the linear Onsager coefficients between the species $i$ and $j$. It is symmetric and positive definite. Furthermore, on the fluid/solid interfaces the no-flux condition is imposed

$$
\mathbf{j}_{i} \cdot v=0 \quad \text { on } \quad \partial \Omega_{p} \backslash \partial \Omega, \quad i=1, \ldots, N .
$$


The electrostatic potential is calculated from Poisson equation with the electric charge density as the bulk source term

$$
\mathscr{E} \Delta \Psi=-e \sum_{j=1}^{N} z_{j} n_{j} \quad \text { in } \quad \Omega_{p},
$$

where $\mathscr{E}$ is the dielectric constant of the solvent. The surface charge $\Sigma$ is assumed to be given at the pores boundaries and the boundary condition reads

$$
\mathscr{E} \nabla \Psi \cdot v=-\Sigma \quad \text { on } \quad \partial \Omega_{p} \backslash \partial \Omega
$$

where $v$ is the unit exterior normal to $\Omega_{p}$.

The various parameters appearing in (102)-(110) are defined in Table 1.

\begin{tabular}{|l|l|l|}
\hline \hline & QUANTITY & CHARACTERISTIC VALUE \\
\hline $\mathrm{e}$ & electron charge & $1.6 \mathrm{e}-19 \mathrm{C}($ Coulomb $)$ \\
\hline$D_{i}^{0}$ & diffusivity of the $i$ th species & $D_{i}^{0} \in(1.333,2.032) \mathrm{e}-09 \mathrm{~m}^{2} / \mathrm{s}$ \\
\hline$k_{B}$ & Boltzmann constant & $1.38 \mathrm{e}-23 \mathrm{~J} / \mathrm{K}$ \\
\hline$n_{c}$ & characteristic concentration & $\left(6.0210^{24}, 6.0210^{26}\right)$ particles $/ \mathrm{m}^{3}$ \\
\hline$T$ & temperature & $293^{\circ} \mathrm{K}(\mathrm{Kelvin})$ \\
\hline $\mathscr{E}$ & dielectric constant & $6.93 \mathrm{e}-10 \mathrm{C} /(\mathrm{mV})$ \\
\hline$\eta$ & dynamic viscosity & $1 \mathrm{e}-3 \mathrm{~kg} /(\mathrm{ms})$ \\
\hline$\ell$ & pore size & $5 \mathrm{e}-9 \mathrm{~m}$ \\
\hline$\lambda_{D}$ & Debye's length & $\sqrt{\mathscr{E} k_{B} T /\left(e^{2} n_{c}\right)} \in(0.042,0.42) \mathrm{nm}$ \\
\hline$z_{j}$ & $j$-th electrolyte valence & given integer \\
\hline$\Sigma$ & surface charge density & $0.129 \mathrm{C} / \mathrm{m}^{2}($ clays $)$ \\
\hline $\mathbf{f}$ & given applied force & $\mathrm{N} / \mathrm{m}^{3}$ \\
\hline$\sigma_{j}$ & $j$-th hard sphere diameter & $2 \mathrm{e}-10 \mathrm{~m}$ \\
\hline$\Psi_{c}$ & characteristic electrokinetic potential & $0.02527 \mathrm{~V}($ Volt $)$ \\
\hline$L_{B}$ & Bjerrum length & $7.3 \mathrm{e}-10 \mathrm{~m}$ \\
\hline
\end{tabular}

Table 1 Data description

The activity coefficients $\gamma_{i}$ and the Onsager coefficients $L_{i j}$ depend on the electrolyte. The large majority of theoretical works are concerned with a simple (socalled ideal) descriptions of charged porous media. It is based on the PoissonNernst-Planck formalism for which the local activity coefficients of ions are neglected and the transport properties are modeled solely from the mobility at infinite dilution. In the ideal description we have

$$
\gamma_{i}=1 \quad \text { and } \quad L_{i j}=\delta_{i j} n_{i} D_{i}^{0} /\left(k_{B} T\right)
$$

where $D_{i}^{0}>0$ is the diffusion coefficient of species $i$ at infinite dilution.

In this section we will suppose that we have an infinite dilution, i.e. an ideal description. 
Remark 10. At finite concentration, the non-ideal effects modify the ion transport and they are to be taken into account if a good quantitative description of the system is required. Different models can be used and a widely accepted model is the Mean Spherical Approximation (MSA) in its simplified form from [31]. It is valid if the diameters of the ions are not too different. The activity coefficients read

$$
\ln \gamma_{j}=-\frac{L_{B} \Gamma z_{j}^{2}}{1+\Gamma \sigma_{j}}+\ln \gamma^{H S}, \quad j=1, \ldots, N
$$

where $\sigma_{j}$ is the $j$-th ion diameter, $L_{B}$ is the Bjerrum length, $\gamma^{H S}$ is the hard sphere term defined by (113), and $\Gamma$ is the MSA screening parameter defined by

$$
\Gamma^{2}=\pi L_{B} \sum_{k=1}^{N} \frac{n_{k} z_{k}^{2}}{\left(1+\Gamma \sigma_{k}\right)^{2}}
$$

For dilute solutions, i.e., when all $n_{j}$ are small, we have

$$
2 \Gamma \approx \kappa=\frac{1}{\lambda_{D}} \quad \text { with } \quad \lambda_{D}=\sqrt{\frac{\mathscr{E} k_{B} T}{e^{2} \sum_{k=1}^{N} n_{k} z_{k}^{2}}},
$$

where $\lambda_{D}$ is the Debye length. Thus, $1 / 2 \Gamma$ generalizes $\lambda_{D}$ at finite concentration and it represents the size of the ionic spheres when the ion diameters $\sigma_{i}$ are different from zero. In (111) $\gamma^{H S}$ is the hard sphere term given by

$$
\ln \gamma^{H S}=p(\xi) \equiv \xi \frac{8-9 \xi+3 \xi^{2}}{(1-\xi)^{3}}, \quad \text { with } \quad \xi=\frac{\pi}{6} \sum_{k=1}^{N} n_{k} \sigma_{k}^{3},
$$

where $\xi$ is the solute packing fraction.

The Onsager coefficients $L_{i j}$ are given by

$$
L_{i j}\left(n_{1}, \ldots, n_{N}\right)=n_{i}\left(\frac{D_{i}^{0}}{k_{B} T} \delta_{i j}+\Theta_{i j}\right)\left(1+\mathscr{R}_{i j}\right), i, j=1, \ldots, N
$$

where $\Theta_{i j}=\Theta_{i j}^{c}+\Theta_{i j}^{H S}$ stands for the hydrodynamic interactions in the MSA formalism. It is divided into two terms: the Coulomb part is

$$
\Theta_{i j}^{c}=-\frac{1}{3 \eta} \frac{z_{i} z_{j} L_{B} n_{j}}{\left(1+\Gamma \sigma_{i}\right)\left(1+\Gamma \sigma_{j}\right)\left(\Gamma+\sum_{k=1}^{N} n_{k} \frac{\pi L_{B} z_{k}^{2} \sigma_{k}}{\left(1+\Gamma \sigma_{k}\right)^{2}}\right)},
$$

and the hard sphere part is

$$
\Theta_{i j}^{H S}=-\frac{\left(\sigma_{i}+\sigma_{j}\right)^{2}}{12 \eta} n_{j} \frac{1-\tilde{Y}_{3} / 5+\left(\tilde{Y}_{3}\right)^{2} / 10}{1+2 \tilde{Y}_{3}},
$$


with

$$
\tilde{Y}_{3}=\frac{\pi}{6} \sum_{i=1}^{N} n_{i} \frac{3 Y_{1} Y_{2}+Y_{3} Y_{0}}{4 Y_{0}^{2}} \quad \text { and } Y_{k}=\frac{\pi}{6} \sum_{i=1}^{N} n_{i} \sigma_{i}^{k}
$$

In (114) $\mathscr{R}_{i j}$ is the electrostatic relaxation term given by

$$
\mathscr{R}_{i j}=\frac{\kappa_{q}^{2} e^{2} z_{i} z_{j}}{3 \mathscr{E} k_{B} T\left(\sigma_{i}+\sigma_{j}\right)\left(1+\Gamma \sigma_{i}\right)\left(1+\Gamma \sigma_{j}\right)} \frac{1-e^{-2 \kappa_{q}\left(\sigma_{i}+\sigma_{j}\right)}}{\kappa_{q}^{2}+2 \Gamma \kappa_{q}+2 \Gamma^{2}-2 \pi L_{B} \sum_{k=1}^{N} n_{k} \frac{z_{k}^{2} e^{-\kappa_{q} \sigma_{k}}}{\left(1+\Gamma \sigma_{k}\right)^{2}}}
$$

where $\kappa_{q}>0$ is defined by

$$
\kappa_{q}^{2}=\frac{e^{2}}{\mathscr{E} k_{\mathrm{B}} T} \frac{\sum_{i=1}^{N} n_{i} z_{i}^{2} D_{i}^{0}}{\sum_{i=1}^{N} D_{i}^{0}}
$$

Note that when the concentrations $n_{j}$ are small, all entries $L_{i j}$ are first order perturbations of the ideal values $\delta_{i j} n_{i} D_{i}^{0} /\left(k_{B} T\right)$ and thus the Onsager tensor is positive at first order.

Remark 11. Homogenization of the non-ideal MSA model was undertaken in [13].

At the outer boundary of the porous medium we set

$$
\Psi+\Psi^{e x t}(x), n_{i}, \mathbf{v} \text { and } p \text { are } \Omega-\text { periodic. }
$$

The applied exterior potential $\Psi^{e x t}(x)$ can typically be linear, equal to $\mathbf{E} \cdot \mathbf{x}$, where $\mathbf{E}$ is an imposed electrical field. Note that the applied exterior force $\mathbf{f}$ in the Stokes equations (103) can also be interpreted as some imposed pressure drop or gravity force. Due to the complexity of the geometry and of the equations, it is necessary for engineering applications to upscale the system (102)-(110), (120) and to replace the flow equations with a Darcy type law, including electro-osmotic effects.

A representative class of porous media are those having a periodic microstructure. We suppose the same periodic microstructure as in subsection 2.2. For such media, and in the ideal case, formal two-scale asymptotic analysis of system (102)(110); (120) has been performed in many previous papers. Many of these works rely on a preliminary linearization of the problem, introduced by O'Brien et al. [64]. Let us mention in particular the work of Looker and Carnie in [48], where the formal two-scale expansions were undertaken and the resulting Onsager relations written explicitly. We will present the rigorous justification of the homogenization result, following article [10]. The numerical experiments are provided in [11]. Other relevant references include [69], [70], [77], [78] and [79] .

Remark 12. in this review we will consider only rigid porous media. In many important applications porous media are deformable. Derivations of the homogenized models for deformable charge porous media were undertaken by Moyne and Murad 
in [57], [58], [59], [60], [61]. For a mathematically rigourous analysis we refer to [14].

The goal of the section is to present the results from [10] and [11], providing the homogenized system for a semi-linearized version of (102)-(110), (120) in a rigid periodic porous medium. The semi-linearization means that we study the solutions being a perturbation of a so-called equilibrium solution which satisfies the full nonlinear system (102)-(110), (120) with vanishing fluxes.

The homogenized system is an elliptic system of $(N+1)$ equations

$$
-\operatorname{div}_{x} \mathscr{M} \nabla\left(p^{0},\left\{\mu_{j}\right\}_{1 \leq j \leq N}\right)=\mathscr{S} \quad \text { in } \Omega,
$$

where $p^{0}$ is the pressure, $\mu_{j}$ the chemical potential of the $j$-th species, $\mathscr{M}$ the Onsager homogenized tensor and $\mathscr{S}$ a source term. Our goal is to derive rigorously equation (121).

Before studying its homogenization, we need a dimensionless form of the equations (102)-(110), (120). We follow the same approach as in [10] and [11]. The known data are listed in Table 1 and concern the characteristic pore size $\ell$, the characteristic domain size $L$, the surface charge density $\Sigma$ (having the characteristic value $\Sigma_{c}$ ), the characteristic concentrations $n_{c}$, the static electrical potential $\Psi^{e x t}$ and the applied fluid force $\mathbf{f}$. As usual, we introduce a small parameter $\varepsilon$ which is the ratio between the pore size and the medium size, $\varepsilon=\ell / L<<1$.

Table 1 permits calculating Debye's length $\lambda_{D}=\sqrt{\mathscr{E} k_{B} T /\left(e^{2} n_{c}\right)}$. Following [42], we introduce the characteristic potential $\zeta=k_{B} T / e$ and the parameter $\beta$ related to the Debye-Hückel parameter $\kappa=1 / \lambda_{D}$, is given by $\beta=\left(\frac{\ell}{\lambda_{D}}\right)^{2}$. Next we rescale the space variable by setting $x^{\prime}=x / L$ (we shall drop the primes for simplicity in the sequel). The pore space becomes $\Omega_{\varepsilon}=\Omega_{p} / L$ which is a periodically perforated domain with period $\varepsilon$. Still following [42], we define other characteristic quantities

$$
\Gamma_{c}=\sqrt{\pi L_{B} n_{c}}, \quad p_{c}=n_{c} k_{B} T, \quad u_{c}=\varepsilon^{2} \frac{k_{B} T n_{c} L}{\eta},
$$

where $p_{c}$ is a pressure equilibrating the electrokinetic forces in (103) and $u_{c}$ is the velocity corresponding to a Poiseuille flow in a tube of diameter $\ell$, length $L$ and pressure drop $p_{c}$. We also introduce adimensionalized forcing terms

$$
\Psi^{e x t, *}=\frac{e \Psi^{e x t}}{k_{B} T}, \quad \mathbf{f}^{*}=\frac{\mathbf{f} L}{p_{c}}, \quad \Sigma^{*}=\frac{\Sigma}{\Sigma_{c}}, \quad N_{\sigma}=\frac{e \Sigma_{c} \ell}{\mathscr{E} k_{B} T},
$$

and adimensionalized unknowns

$$
p^{\varepsilon}=\frac{p}{p_{c}}, \mathbf{v}^{\varepsilon}=\frac{\mathbf{v}}{u_{c}}, \Psi^{\varepsilon}=\frac{e \Psi}{k_{B} T}, n_{j}^{\varepsilon}=\frac{n_{j}}{n_{c}}, \mathbf{j}_{j}^{\varepsilon}=\frac{\mathbf{j}_{j} L}{n_{c} D_{j}^{0}} .
$$

The dimensionless equations for hydrodynamical and electrostatic part are thus 


$$
\begin{gathered}
\varepsilon^{2} \Delta \mathbf{v}^{\varepsilon}-\nabla p^{\varepsilon}=\mathbf{f}^{*}+\sum_{j=1}^{N} z_{j} n_{j}^{\varepsilon}(x) \nabla \Psi^{\varepsilon} \quad \text { in } \Omega_{\varepsilon}, \\
\mathbf{v}^{\varepsilon}=0 \quad \text { on } \quad \partial \Omega_{\varepsilon} \backslash \partial \Omega, \quad \operatorname{div} \mathbf{v}^{\varepsilon}=0 \quad \text { in } \Omega_{\varepsilon}, \\
-\varepsilon^{2} \Delta \Psi^{\varepsilon}=\beta \sum_{j=1}^{N} z_{j} n_{j}^{\varepsilon}(x) \quad \text { in } \Omega_{\varepsilon}, \\
\varepsilon \nabla \Psi^{\varepsilon} \cdot v=-N_{\sigma} \Sigma^{*} \text { on } \partial \Omega_{\varepsilon} \backslash \partial \Omega, \\
\left(\Psi^{\varepsilon}+\Psi^{e x t, *}\right), \quad n_{i}^{\varepsilon}, \quad \mathbf{v}^{\varepsilon} \text { and } p^{\varepsilon} \quad \text { are } \Omega-\text { periodic in } x, \\
\operatorname{div}\left(\mathbf{j}_{i}^{\varepsilon}+\mathbf{P e}_{i} n_{i}^{\varepsilon} \mathbf{v}^{\varepsilon}\right)=0 \quad \text { in } \quad \Omega_{\varepsilon}, i=1, \ldots, N, \\
\mathbf{j}_{i}^{\varepsilon} \cdot v=0 \text { on } \partial \Omega_{\varepsilon} \backslash \partial \Omega, i=1, \ldots, N, \\
\mathbf{j}_{i}^{\varepsilon}=-n_{i}^{\varepsilon} \nabla M_{i}^{\varepsilon} \quad \text { and } \quad M_{i}^{\varepsilon}=\ln \left(n_{i}^{\varepsilon} e^{z_{i}} \Psi^{\varepsilon}\right), i=1, \ldots, N,
\end{gathered}
$$

where the Péclet number for the $i$-th species is $\mathrm{Pe}_{i}=\frac{u_{c} L}{D_{i}^{0}}=O(1)$.

Remark 13. Existence results for a coupled Navier-Stokes-Nernst-Planck- Poisson system are in [76].

Remark 14. After writing the dimensionless form, we are able to precise in which sense the non-ideal MSA model from remark $\pi^{10}$ is close to the ideal case. The small parameter is the characteristic value $\xi_{c}=\frac{\pi}{6} n_{c} \sigma_{c}^{3}$ of the solute packing fraction, where $\sigma_{c}$ is the characteristic ion diameter. In [13] it was established that, under the hypothesis that

$$
\left.b i=\frac{L_{B}}{\sigma_{c}} \text { (Bjerrum's parameter }\right) \quad \text { and } \quad S=\frac{k_{B} T}{\eta D_{c}^{0} \sigma_{c}}
$$

are $O(1)$, then the ideal case model is the vanishing solute packing fraction $\xi_{c}$ limit of our non-ideal MSA model. Note that small $\xi_{c}$ means a low concentration, weighted by the ion size. Namely, we have

$$
\left(\frac{D_{i}^{0}}{k_{B} T} \delta_{i j}+\Theta_{i j}\right)\left(1+\mathscr{R}_{i j}\right)=\delta_{i j}+O\left(\sqrt{\xi_{c}}\right), \quad \text { and } \quad \ln \gamma_{j}^{\varepsilon}=O\left(\sqrt{\xi_{c}}\right)
$$

\subsection{Equilibrium solution}

The goal of this subsection is to find a so-called equilibrium solution of system (122)-(129) when the exterior forces are vanishing $\mathbf{f}=0$ and $\Psi^{e x t}=0$. However, the surface charge density $\Sigma^{*}$ is not assumed to vanish or to be small. This equilibrium solution will be a reference solution around which we shall linearize system (122)(129) in the next subsection. 
Then we perform the homogenization of the (partially) linearized system. We denote by $n_{i}^{0, \varepsilon}, \Psi^{0, \varepsilon}, \mathbf{v}^{0, \varepsilon}, M_{i}^{0, \varepsilon}, p^{0, \varepsilon}$ the equilibrium quantities.

In the case $\mathbf{f}=0$ and $\Psi^{\text {ext }}=0$, one can find an equilibrium solution by choosing a zero fluid velocity and taking all diffusion fluxes equal to zero. More precisely, we require

$$
\mathbf{v}^{0, \varepsilon}=0 \quad \text { and } \quad \nabla M_{j}^{0, \varepsilon}=0
$$

which obviously implies that $\mathbf{j}_{i}^{0, \varepsilon}=0$ and (127)-(128) are satisfied. From $\nabla M_{j}^{0, \varepsilon}=0$ and (129) we deduce that there exists constant $n_{j}^{0}(\infty)>0$ such that

$$
n_{j}^{0, \varepsilon}(x)=n_{j}^{0}(\infty) \exp \left\{-z_{j} \Psi^{0, \varepsilon}(x)\right\} .
$$

The Stokes equation (122) shall give the corresponding value of the pressure satisfying

$$
\nabla p^{0, \varepsilon}(x)=-\sum_{j=1}^{N} z_{j} n_{j}^{0, \varepsilon}(x) \nabla \Psi^{0, \varepsilon}(x) \Rightarrow p^{0, \varepsilon}(x)=\sum_{j=1}^{N} n_{j}^{0}(\infty)(x) e^{-z_{j} \Psi^{0, \varepsilon}(x)} .
$$

The value $n_{j}^{0}(\infty)$ is the reservoir concentration (also called the infinite dilute concentration) which will be later assumed to satisfy the bulk electroneutrality condition for zero potential.

Then the electrostatic equation (124) reduces to the Poisson-Boltzmann equation which is a nonlinear partial differential equation for the sole unknown $\Psi^{0, \varepsilon}$

$$
\left\{\begin{array}{l}
-\varepsilon^{2} \Delta \Psi^{0, \varepsilon}=\beta \sum_{j=1}^{N} z_{j} n_{j}^{0}(\infty) \exp \left\{-z_{j} \Psi^{0, \varepsilon}\right\} \text { in } \Omega_{\varepsilon} \\
\varepsilon \nabla \Psi^{0, \varepsilon} \cdot v=-N_{\sigma} \Sigma^{*}\left(\frac{x}{\varepsilon}\right) \text { on } \partial \Omega_{\varepsilon} \backslash \partial \Omega, \Psi^{0, \varepsilon} \text { is } \Omega \text { - periodic. }
\end{array}\right.
$$

We note that problem (133) is equivalent to the following minimization problem:

$$
\inf _{\varphi \in V_{\varepsilon}} J_{\varepsilon}(\varphi)
$$

with $V_{\varepsilon}=\left\{\varphi \in H^{1}\left(\Omega_{\varepsilon}\right), \varphi\right.$ is $\Omega$ - periodic $\}$ and

$$
J_{\varepsilon}(\varphi)=\frac{\varepsilon^{2}}{2} \int_{\Omega_{\varepsilon}}|\nabla \varphi|^{2} d x+\beta \sum_{j=1}^{N} \int_{\Omega_{\varepsilon}} n_{j}^{0}(\infty) e^{-z_{j} \phi} d x+\varepsilon N_{\sigma} \int_{\Gamma^{\varepsilon}} \Sigma^{*}\left(\frac{x}{\varepsilon}\right) \varphi d S
$$

The functional $J_{\varepsilon}$ is strictly convex, which gives the uniqueness of the minimizer. Nevertheless, for arbitrary non-negative $\beta, n_{j}^{0}(\infty)$ and $N_{\sigma}, J_{\varepsilon}$ may be not coercive on $V_{\varepsilon}$ if all $z_{j}$ 's have the same sign (take $\varphi$ to be constant, of the same sign than the $z_{j}$ 's and going to infinity). Therefore, we must put a condition on the $z_{j}$ 's so that the minimization problem (134) admits a solution. Following the literature, we impose the bulk electroneutrality condition 


$$
\sum_{j=1}^{N} z_{j} n_{j}^{0}(\infty)=0
$$

which guarantees that for $\Sigma^{*}=0$, the unique solution is $\Psi^{0, \varepsilon}=0$. Under (135) it is easy to see that $J_{\varepsilon}$ is coercive on $V_{\varepsilon}$.

We recall that we suppose a periodic porous medium as introduced in subsection 2.2. By the uniqueness, $\Psi^{0, \varepsilon}(x)=\Psi^{0}(x / \varepsilon)$, where $\Psi^{0}(y)$ is a solution to the problem

$$
\inf _{\varphi \in V} J(\varphi)
$$

with $V=\left\{\varphi \in H^{1}\left(Y_{F}\right), \varphi\right.$ is $1-$ periodic $\}$ and

$$
J(\varphi)=\frac{1}{2} \int_{Y_{F}}\left|\nabla_{y} \varphi(y)\right|^{2} d y+\beta \sum_{j=1}^{N} \int_{Y_{F}} n_{j}^{c} \exp \left\{-z_{j} \varphi\right\} d y+N_{\sigma} \int_{S} \Sigma^{*}(y) \varphi d S .
$$

Note that $J$ is strictly convex, which gives the uniqueness of the minimizer. Under condition (135) it is easy to see that $J$ is coercive on $V$.

Next difficulty is with the continuity of the functional $J$. In fact it is not defined on $V$, but on its proper subspace $V_{1}=\left\{\varphi \in H^{1}\left(Y_{F}\right), \exp \left\{\max _{j}\left|z_{j}\right||\varphi|\right\} \in L^{1}\left(Y_{F}\right)\right\}$. This situation complicates the solvability of the problem (136). The corresponding existence result was established in [47], using a penalization, with a cut-off of the nonlinear terms and applying the theory of pseudo-monotone operators. It reads as follows:

Lemma 5 ([47]). Assume that the bulk electroneutrality condition (135) holds true and $\Sigma^{*} \in L^{2}(S)$. Then problem (136) has a unique solution $\Psi^{0} \in V$ such that

$$
\sum_{j=1}^{N} z_{j} e^{-z_{j} \Psi^{0}} \in L^{1}\left(Y_{F}\right) \quad \text { and } \quad \Psi^{0} \sum_{j=1}^{N} z_{j} e^{-z_{j} \Psi^{0}} \in L^{1}\left(Y_{F}\right)
$$

We need that $n_{j}^{0}=n_{j}^{c} \exp \left\{-z_{j} \Psi^{0}\right\}$ satisfies the lower bound $n_{j}^{0}(y) \geq C>0$ in $Y_{F}$. It is a consequence of the $L^{\infty}$-estimate for $\Psi^{0}$ from [12], proved by using elementary comparison arguments (a similar result is also proved in [36]).

It is based on the comparison with the following auxiliary Neumann problem

$$
\left\{\begin{array}{l}
-\Delta U=\frac{1}{\left|Y_{F}\right|} \int_{S} \Sigma^{*} d S \text { in } Y_{F} \\
\nabla U \cdot v=-\Sigma^{*} \text { on } S \\
U \text { is } 1-\text { periodic }, \quad \int_{Y_{F}} U(y) d y=0
\end{array}\right.
$$

Problem (137) admits a unique solution $U \in H_{p e r}^{1}\left(Y_{F}\right) \cap C\left(\bar{Y}_{F}\right)$. If $\Sigma^{*}$ and $S$ are $C^{\infty}$, then $U$ is $C^{\infty}$ as well. $U$ achieves its minimum and maximum in $\overline{Y_{F}}$.

The $L^{\infty}$-bound for $\Psi^{0}$ reads as follows

Proposition 15. (see [12]) The solution $\Psi^{0}$ of (136) satisfies the following bounds 


$$
\begin{gathered}
U(y)-U_{m}-\frac{1}{z_{1}} \log \max \left(1, \frac{\bar{\sigma}}{\beta z_{1} n_{1}^{0}(\infty)}-\sum_{j \in j^{+}} \frac{z_{j} n_{j}^{0}(\infty)}{z_{1} n_{1}^{0}(\infty)}\right) \geq \Psi^{0}(y) \geq \\
U(y)-U_{M}-\frac{1}{z_{N}} \log \max \left(1, \frac{\bar{\sigma}}{\beta z_{N} n_{N}^{0}(\infty)}-\sum_{j \in j^{-}} \frac{z_{j} n_{j}^{0}(\infty)}{z_{N} n_{N}^{0}(\infty)}\right),
\end{gathered}
$$

where the symbols $j^{+}$and $j^{-}$denote the sets of positive and negative valences, respectively, and

$$
\bar{\sigma}=\frac{1}{\left|Y_{F}\right|} \int_{S} \Sigma^{*} d S, \quad U_{m}=\min _{y \in \overline{Y_{F}}} U(y) \text { and } U_{M}=\max _{y \in \overline{Y_{F}}} U(y) .
$$

By classical regularity theory for elliptic partial differential equations, we easily deduce that for $S \in C^{\infty}$ and $\sigma \in C_{p e r}^{\infty}(S), \Psi^{0} \in C^{\infty}\left(\bar{Y}_{F}\right)$.

Remark 15. In [12] the asymptotic analysis of (136), when $\beta$ goes to zero, was undertaken. This case corresponds to very small pores, $L<<\lambda_{D}$. The asymptotic regime depends on the sign of the averaged charge $\int_{S} \Sigma^{*} d S$. If it is negative (which means that the surface is positively charged), then only the anion with the most negative valence $\left(z_{1}\right)$ is important and that the potential behaves as

$$
\Psi^{0} \approx \frac{\log \beta}{z_{1}}+\varphi_{0}
$$

where $\varphi_{0}$ is the solution of the reduced system, involving only the species 1 ,

$$
\left\{\begin{array}{l}
\Delta \varphi_{0}=-z_{1} n_{1}^{0}(\infty) e^{-z_{1} \varphi_{0}} \text { in the bulk } Y_{F}, \\
\nabla \varphi_{0} \cdot v=-\Sigma^{*}(y) \text { on the surface } S .
\end{array}\right.
$$

As a consequence, the cation concentrations go to zero while the ion concentrations blow up as $n_{j}=O\left(\beta^{-z_{j} / z_{1}}\right)$ and $n_{1}>>n_{j}$ for $j \neq 1$.

Remark 16. The opposite situation, when $\beta$ goes to infinity, was also addressed in [12]. This scaling corresponds to very large pores, $L>>\lambda_{D}$. The Debye's layer, describing the behavior of the solution close to the surface, was constructed in a general geometric setting and a rigorous error estimate was given. If we choose the characteristic concentration $n_{c}=\sum_{k=1}^{N} z_{k}^{2} n_{k}^{0}(\infty)$, then $\sum_{k=1}^{N} z_{k}^{2} n_{k}^{0}(\infty)=1$ and locally, close to the surface, the potential behaves as

$$
\Psi(y) \approx \frac{-\Sigma^{*}}{\sqrt{\beta}} \exp \{-d(y) \sqrt{\beta}\}
$$

where $d(y)$ is the distance between the point $y$ and the surface. Away from the surface, the concentrations $n_{j}$ are constant and satisfy the so-called bulk electroneutrality condition.

The boundary condition for the electrostatic interaction between the two phases is very often simplified by replacing surface charge $\Sigma^{*}$, which corresponds to the 
chemistry of the system, by a surface potential. Its boundary value at the no slip plane is known as the zeta potential $\zeta$. In [12] the asymptotic behavior for large $\beta$ was established. It is again a boundary layer but with a totally different profile. More precisely we establish

$$
\Psi(y) \approx \Psi_{0, \zeta}(\sqrt{\beta} d(y))
$$

where $d(y)$ is the distance between the point $y$ and the surface and $\Psi_{0, \zeta}$ is the solution of the nonlinear ordinary differential equation

$$
\left\{\begin{array}{l}
\left.\Psi_{0, \zeta}\right|_{\xi=0}=\zeta, \quad \mathscr{C}(x)=\sum_{j=1}^{N} n_{j}^{0}(\infty) e^{-z_{j} x} \\
\frac{d}{d \xi} \Psi_{0, \zeta}=-2 \operatorname{sign}(\zeta) \sqrt{\mathscr{C}\left(\Psi_{0, \zeta}\right)-\mathscr{C}(0)} .
\end{array}\right.
$$

which, starting from the boundary value $\zeta$ on the surface, is exponentially decaying at infinity. In many situations, the explicit solutions for $\Psi_{0, \zeta}$ are known. For example, in the case $-z_{1}=1=z_{2}$ and $n_{1}^{0}(\infty)=n_{2}^{0}(\infty)=1 / 2$, we have the following GouyChapman solution

$$
\Psi_{0, \zeta}\left(q^{\prime}, \xi\right)=2 \ln \frac{1+\tanh (\zeta / 2) e^{-\xi}}{1-\tanh (\zeta / 2) e^{-\xi}} .
$$

Hence in the case of given potential at the boundary the normal component of the electrical field will behave as $\sqrt{\beta}$, which is unrealistic. In fact, it is rather the surface charge density $\Sigma$, proportional to the normal derivative of $\Psi$, than $\zeta$, which is the relevant parameter for the physical modeling.

\subsection{Linearization and the a priori estimates for the perturbation}

We now proceed to the linearization of electrokinetic equations (122)-(129) around the equilibrium solution computed in subsection 5.1. We therefore assume that the external forces, namely the static electric potential $\Psi^{e x t}(x)$ and the hydrodynamic force $\mathbf{f}(x)$, are small. Note that the surface charge density $\Sigma^{*}$ on the pore walls $\Gamma^{\mathcal{E}}$ need not to be small since it is part of the equilibrium problem. Such a linearization process is classical in the ideal case (see the seminal paper [64] by O'Brien et al.). For small exterior forces, we write the perturbed electrokinetic unknowns as

$$
\begin{array}{ccc}
n_{i}^{\varepsilon}(x)=n_{i}^{0, \varepsilon}(x)+\delta n_{i}^{\varepsilon}(x), & \Psi^{\varepsilon}(x)=\Psi^{0, \varepsilon}(x)+\delta \Psi^{\varepsilon}(x), \\
\mathbf{v}^{\varepsilon}(x)=\mathbf{v}^{0, \varepsilon}(x)+\delta \mathbf{v}^{\varepsilon}(x), & p^{\varepsilon}(x)=p^{0, \varepsilon}(x)+\delta p^{\varepsilon}(x),
\end{array}
$$

where $n_{i}^{0, \varepsilon}, \Psi^{0, \varepsilon}, \mathbf{v}^{0, \varepsilon}, p^{0, \varepsilon}$ are the equilibrium quantities, corresponding to $\mathbf{f}=0$ and $\Psi^{e x t}=0$. The $\delta$ prefix indicates a perturbation. Since the equilibrium velocity vanishes $\mathbf{v}^{0, \varepsilon}=0$, we identify in the sequel $\mathbf{v}^{\varepsilon}=\delta \mathbf{v}^{\varepsilon}$. 
Motivated by the form of the Boltzmann equilibrium distribution and the calculation of $n_{i}^{0, \varepsilon}$, we follow the lead of [64] and introduce a so-called ionic potential $\Phi_{i}^{\varepsilon}$ which is defined in terms of $n_{i}^{\varepsilon}$ by

$$
n_{i}^{\varepsilon}(x)=n_{i}^{0}(\infty) \exp \left\{-z_{i}\left(\Psi^{\varepsilon}(x)+\Phi_{i}^{\varepsilon}(x)+\Psi^{e x t}, *(x)\right)\right\},
$$

After linearization (140) yields

$$
\delta n_{i}^{\varepsilon}(x)=-z_{i} n_{i}^{0, \varepsilon}(x)\left(\delta \Psi^{\varepsilon}(x)+\Phi_{i}^{\varepsilon}(x)+\Psi^{e x t, *}(x)\right) .
$$

Introducing (141) into (122)-(127) and linearizing yields the following equations for $\delta \Psi^{\varepsilon}, \delta \mathbf{v}^{\varepsilon}, \delta p^{\varepsilon}$ and $\Phi_{i}^{\varepsilon}$

$$
\begin{gathered}
-\varepsilon^{2} \Delta\left(\delta \Psi^{\varepsilon}\right)+\beta\left(\sum_{j=1}^{N} z_{j}^{2} n_{j}^{0, \varepsilon}(x)\right) \delta \Psi^{\varepsilon}= \\
-\beta\left(\sum_{j=1}^{N} z_{j}^{2} n_{j}^{0, \varepsilon}(x)\left(\Phi_{j}^{\varepsilon}+\Psi^{e x t, *}\right)\right) \text { in } \Omega_{\varepsilon}, \\
\varepsilon \nabla \delta \Psi^{\varepsilon} \cdot v=0 \text { on } \partial \Omega_{\varepsilon} \backslash \partial \Omega \\
\delta \Psi^{\varepsilon}(x)+\Psi^{e x t, *}(x) \text { is } \Omega-\text { periodic, } \\
\varepsilon^{2} \Delta \delta \mathbf{v}^{\varepsilon}-\nabla\left(\delta p^{\varepsilon}+\sum_{j=1}^{N} z_{j} n_{j}^{0, \varepsilon}\left(\delta \Psi^{\varepsilon}+\Phi_{j}^{\varepsilon}+\Psi^{e x t, *}\right)\right)= \\
\mathbf{f}^{*}-\sum_{j=1}^{N} z_{j} n_{j}^{0, \varepsilon}(x)\left(\nabla \Phi_{j}^{\varepsilon}+\mathbf{E}^{*}\right) \text { in } \Omega_{\varepsilon}, \\
\operatorname{div} \delta \mathbf{v}^{\varepsilon}=0 \quad \text { in } \quad \Omega_{\varepsilon}, \quad \delta \mathbf{v}^{\varepsilon}=0 \quad \text { on } \partial \Omega_{\varepsilon} \backslash \partial \Omega \\
\delta \mathbf{v}^{\varepsilon} \quad \text { and } \delta p^{\varepsilon} \text { are } \Omega-\text { periodic. }
\end{gathered}
$$

Note that the perturbed velocity is actually equal to the overall velocity and that it is convenient to introduce a global pressure $P^{\varepsilon}$

$$
\delta \mathbf{v}^{\varepsilon}=\mathbf{v}^{\varepsilon}, P^{\varepsilon}=\delta p^{\varepsilon}+\sum_{j=1}^{N} z_{j} n_{j}^{0, \varepsilon}\left(\delta \Psi^{\varepsilon}+\Phi_{j}^{\varepsilon}+\Psi^{e x t, *}\right)
$$

A straightforward calculation yields for $\Phi_{j}^{\varepsilon}$

$$
\begin{gathered}
\operatorname{div}\left(n_{j}^{0, \varepsilon}(x)\left(\nabla \Phi_{j}^{\varepsilon}+\mathbf{E}^{*}+\frac{\mathbf{P} \mathbf{e}_{j}}{z_{j}} \mathbf{v}^{\varepsilon}\right)\right)=0 \text { in } \Omega_{\varepsilon}, \\
\left(\nabla \Phi_{j}^{\varepsilon}+\mathbf{E}^{*}\right) \cdot v=0 \text { on } \partial \Omega_{\varepsilon} \backslash \partial \Omega \\
\Phi_{j}^{\varepsilon} \text { is } \Omega \text { - periodic. }
\end{gathered}
$$

$\delta \Psi^{\varepsilon}$ does not enter equations (145)-(147), (149)-(151) and thus is decoupled from the main unknowns $\mathbf{v}^{\varepsilon}, P^{\varepsilon}$ and $\Phi_{i}^{\varepsilon}$. The system (132), (133), (145)-(148), (149)- 
(151) is the same microscopic linearized system for the ionic transport as in the work of Looker and Carnie [48].

Next, we establish the variational formulation of system (145)-(147), (149)-(151) for the unknowns $\left\{\mathbf{v}^{\varepsilon}, P^{\varepsilon},\left\{\Phi_{j}^{\varepsilon}\right\}_{j=1, \ldots, N}\right\}$ and prove that it admits a unique solution. The functional spaces related to the velocity field are

$$
W^{\varepsilon}=\left\{\mathbf{g} \in H^{1}\left(\Omega_{\varepsilon}\right)^{3}, \mathbf{g}=0 \text { on } \partial \Omega_{\varepsilon} \backslash \partial \Omega, \Omega-\text { periodic in } x\right\}
$$

and

$$
H^{\varepsilon}=\left\{\mathbf{g} \in W^{\varepsilon}, \operatorname{div} \mathbf{g}=0 \text { in } \Omega_{\varepsilon}\right\} .
$$

The variational formulation of (145)-(151) is:

Find $\mathbf{v}^{\varepsilon} \in H^{\varepsilon}$ and $\left\{\Phi_{j}^{\varepsilon}\right\}_{j=1, \ldots, N} \in H^{1}\left(\Omega_{\varepsilon}\right)^{N}, \Phi_{j}^{\varepsilon}$ being $\Omega$-periodic, such that, for any test functions $\mathbf{g} \in H^{\varepsilon}$ and $\mathbf{b} \in H^{1}\left(\Omega_{\varepsilon}\right)^{N}$, b being $\Omega$-periodic,

$$
a\left(\left(\mathbf{v}^{\varepsilon},\left\{\Phi_{j}^{\varepsilon}\right\}\right),(\mathbf{g}, \mathbf{b})\right)=\langle\mathscr{L},(\mathbf{g}, \mathbf{b})\rangle
$$

where the bilinear form $a$ and the linear form $\mathscr{L}$ are defined by

$$
\begin{gathered}
a\left(\left(\mathbf{v}^{\varepsilon},\left\{\Phi_{j}^{\varepsilon}\right\}\right),(\mathbf{g}, \mathbf{b})\right):=\varepsilon^{2} \int_{\Omega_{\varepsilon}} \nabla \mathbf{v}^{\varepsilon}: \nabla \mathbf{g} d x+\sum_{i=1}^{N} \frac{z_{i}^{2}}{\mathrm{Pe}_{i}} \int_{\Omega_{\varepsilon}} n_{i}^{0, \varepsilon} \nabla \Phi_{i}^{\varepsilon} \cdot \nabla b_{i} d x \\
\quad+\sum_{j=1}^{N} z_{j} \int_{\Omega_{\varepsilon}} n_{j}^{0, \varepsilon}\left(\mathbf{v}^{\varepsilon} \cdot \nabla b_{j}-\mathbf{g} \cdot \nabla \Phi_{j}^{\varepsilon}\right) d x \\
\langle\mathscr{L},(\mathbf{g}, \mathbf{b})\rangle:=\sum_{i=1}^{N} z_{i} \int_{\Omega_{\varepsilon}} n_{i}^{0, \varepsilon} \mathbf{E}^{*} \cdot\left(\mathbf{g}-\frac{z_{i}}{\mathrm{Pe}_{i}} \nabla \phi_{i}\right) d x-\int_{\Omega_{\varepsilon}} \mathbf{f}^{*} \cdot \mathbf{v} d x
\end{gathered}
$$

where, for simplicity, we denote by $\mathbf{E}^{*}$ the electric field corresponding to the potential $\Psi^{e x t, *}$, i.e., $\mathbf{E}^{*}(x)=\nabla \Psi^{e x t, *}(x)$.

Lemma 6. (see [10]) Let $\mathbf{E}^{*}$ and $\mathbf{f}^{*}$ be given elements of $L^{2}(\Omega)^{3}$. The variational formulation (152)-(153) admits a unique solution $\left(\mathbf{v}^{\varepsilon},\left\{\Phi_{j}^{\varepsilon}\right\}\right) \in H^{\varepsilon} \times H^{1}\left(\Omega_{\varepsilon}\right)^{3}$, such that $\Phi_{j}^{\varepsilon}$ are 1-periodic and $\int_{\Omega_{\varepsilon}} \Phi_{j}^{\varepsilon}(x) d x=0$. Furthermore, there exists a positive constant $C$, independent of $\varepsilon$, such that

$$
\begin{gathered}
\left\|\mathbf{v}^{\varepsilon}\right\|_{L^{2}\left(\Omega_{\varepsilon}\right)^{3}}+\varepsilon\left\|\nabla \mathbf{v}^{\varepsilon}\right\|_{L^{2}\left(\Omega_{\varepsilon}\right)^{9}}+\max _{1 \leq j \leq N}\left\|\Phi_{j}^{\varepsilon}\right\|_{H^{1}\left(\Omega_{\varepsilon}\right)} \leq \\
C\left(\left\|\mathbf{E}^{*}\right\|_{L^{2}(\Omega)^{3}}+\left\|\mathbf{f}^{*}\right\|_{L^{2}(\Omega)^{3}}\right) .
\end{gathered}
$$

Note that the a priori estimates (154) follow by testing the problem (152)-(153) by the solution, using the $L^{\infty}$-estimate for $\Psi^{0}$ and using the well-known scaled Poincaré inequality in $\Omega_{\varepsilon}(7)$.

In order to use the two-scale convergence from section 3 , we need that our unknowns are $\left(\mathbf{v}^{\varepsilon}, P^{\varepsilon},\left\{\Phi_{j}^{\varepsilon}\right\}\right)$ are defined on $\Omega$. As in section $4, \mathbf{v}^{\varepsilon}$ is extended by zero to $\Omega \backslash \Omega_{\varepsilon}$. The pressure field is reconstructed using de Rham's theorem and extended by formula (60) from section 4 to $\tilde{P}^{\varepsilon}$, being uniformly bounded, with re- 
spect to $\varepsilon$, in $L_{0}^{2}(\Omega)$. For $\left\{\Phi_{j}^{\varepsilon}\right\}$ we use an extension operator from the perforated domain $\Omega^{\varepsilon}$ into $\Omega$. As was proved in [1], under the assumptions on the geometry from subsection 2.2, there exists such an extension operator $T^{\varepsilon}$ from $H^{1}\left(\Omega_{\varepsilon}\right)$ in $H^{1}(\Omega)$ satisfying $\left.T^{\varepsilon} \phi\right|_{\Omega^{\varepsilon}}=\phi$ and the inequalities

$$
\left\|T^{\varepsilon} \phi\right\|_{L^{2}(\Omega)} \leq C\|\phi\|_{L^{2}\left(\Omega_{\varepsilon}\right)},\left\|\nabla\left(T^{\varepsilon} \phi\right)\right\|_{L^{2}(\Omega)} \leq C\|\nabla \phi\|_{L^{2}\left(\Omega_{\varepsilon}\right)}
$$

with a constant $C$ independent of $\varepsilon$, for any $\phi \in H^{1}\left(\Omega_{\varepsilon}\right)$. We keep for the extended function $T^{\varepsilon} \Phi_{j}^{\varepsilon}$ the same notation $\Phi_{j}^{\varepsilon}$.

Hence the extensions satisfy estimates (154).

\subsection{Homogenization via the two-scale convergence}

The formal two-scale asymptotic expansion method from sections 1 and 2 can be applied to system (145)-(147), (149)-(151), as in [48] and [10]. Introducing the fast variable $y=x / \varepsilon$, it assumes that the solution of (145)-(147), (149)-(151) is given by

$$
\left\{\begin{array}{l}
\mathbf{v}^{\varepsilon}(x)=\mathbf{v}^{0}(x, x / \varepsilon)+\varepsilon \mathbf{v}^{1}(x, x / \varepsilon)+\ldots \\
\tilde{P}^{\varepsilon}(x)=p^{0}(x)+\varepsilon p^{1}(x, x / \varepsilon)+\ldots \\
\Phi_{j}^{\varepsilon}(x)=\Phi_{j}^{0}(x)+\varepsilon \Phi_{j}^{1}(x, x / \varepsilon)+\ldots
\end{array}\right.
$$

We then plug this ansatz in the equations (145)-(147), (149)-(151). In the way analogous to the calculations in section 2 , we identify the various powers of $\varepsilon$ and obtain a cascade of equations from which we retain only the leading ones that constitute the two-scale homogenized problem. For details we refer to [48]. We will present a rigorous passing to the limit using the two-scale convergence from section 3 .

Lemma 6 and the two-scale compactness Proposition 4 from section 3 imply

Theorem 2. (see [10]) Under the assumptions of Lemma 6, there exists

$$
\begin{gathered}
\left(\mathbf{v}^{0}, p^{0}\right) \in L^{2}\left(\Omega ; H_{p e r}^{1}(Y)^{3}\right) \times L_{0}^{2}(\Omega) \quad \text { and } \\
\left\{\Phi_{j}^{0}, \Phi_{j}^{1}\right\}_{j=1, \ldots, N} \in\left(H^{1}(\Omega) \times L^{2}\left(\Omega ; H_{p e r}^{1}(Y)\right)\right)^{N}
\end{gathered}
$$

such that for a subsequence, denoted by the same indices, the solution of (145)(147), (149)-(151) converges in the following sense 


$$
\begin{gathered}
\mathbf{v}^{\varepsilon} \rightarrow \mathbf{v}^{0}(x, y) \quad \text { in the two-scale sense } \\
\varepsilon \nabla \mathbf{v}^{\varepsilon} \rightarrow \nabla_{y} \mathbf{v}^{0}(x, y) \quad \text { in the two-scale sense } \\
\tilde{P}^{\varepsilon} \rightarrow p^{0}(x) \quad \text { strongly in } L^{2}(\Omega)
\end{gathered}
$$

Next we rewrite the variational problem (152)-(153) in the equivalent form, where the velocity test function are not divergence-free and the pressure term is explicitly present:

$$
\begin{gathered}
\varepsilon^{2} \int_{\Omega_{\varepsilon}} \nabla \mathbf{v}^{\varepsilon}: \nabla \xi d x-\int_{\Omega_{\varepsilon}} p^{\varepsilon} \operatorname{div} \xi d x+\sum_{j=1}^{N} \int_{\Omega_{\varepsilon}} z_{j} n_{j}^{0, \varepsilon}\left(-\xi \cdot \nabla \Phi_{j}^{\varepsilon}+\mathbf{v}^{\varepsilon} \cdot \nabla b_{j}\right) d x+ \\
\sum_{j=1}^{N} \frac{z_{j}^{2}}{\mathrm{Pe}_{j}} \int_{\Omega_{\varepsilon}} n_{j}^{0, \varepsilon} \nabla \Phi_{j}^{\varepsilon} \cdot \nabla b_{j} d x=-\sum_{j=1}^{N} \frac{z_{j}^{2}}{\mathrm{Pe}_{j}} \int_{\Omega_{\varepsilon}} n_{j}^{0, \varepsilon} \mathbf{E}^{*} \cdot \nabla b_{j} d x \\
+\sum_{j=1}^{N} \int_{\Omega_{\varepsilon}} z_{j} n_{j}^{0, \varepsilon} \mathbf{E}^{*} \cdot \xi d x-\int_{\Omega_{\varepsilon}} \mathbf{f}^{*} \cdot \xi d x
\end{gathered}
$$

for any test functions $\xi \in W^{\varepsilon}$ and $\mathbf{g} \in H^{1}\left(\Omega_{\varepsilon}\right)^{N}, b_{j}$ being 1-periodic, $1 \leq j \leq N$. We keep the divergence constraint $\operatorname{div} \mathbf{v}^{\varepsilon}=0$ in $\Omega_{\varepsilon}$. Next we define the two-scale test functions:

$$
\begin{gathered}
\xi^{\varepsilon}(x)=\xi\left(x, \frac{x}{\varepsilon}\right), \quad \xi \in C_{p e r}^{\infty}\left(\Omega ; H_{p e r}^{1}(Y)^{3}\right), \\
\xi=0 \text { on } \Omega \times S, \quad \operatorname{div}_{y} \xi(x, y)=0 \text { on } \Omega \times Y, \\
b_{j}^{\varepsilon}(x)=\varphi_{j}(x)+\varepsilon \gamma_{j}\left(x, \frac{x}{\varepsilon}\right), \varphi_{j} \in C_{p e r}^{\infty}(\Omega), \quad \gamma_{j} \in C_{p e r}^{\infty}\left(\Omega ; H_{p e r}^{1}\left(Y_{F}\right)\right) .
\end{gathered}
$$

We take as test function in equation $(156)\left(\xi^{\varepsilon}, \mathbf{b}^{\varepsilon}\right)$. Now we can pass to the limit in (156), along the same lines as in section 4 . For the solution we use the convergences from Theorem 2. After passing to the two-scale limit in (156) we get that the limit $\left(\mathbf{v}^{0}, p^{0},\left\{\Phi_{j}^{0}, \Phi_{j}^{1}\right\}\right)$ satisfy the following two-scale variational formulation:

Theorem 3. Let

$$
\begin{gathered}
\left(\mathbf{v}^{0}, p^{0}\right) \in L^{2}\left(\Omega ; H_{p e r}^{1}(Y)^{3}\right) \times L_{0}^{2}(\Omega) \quad \text { and } \\
\left\{\Phi_{j}^{0}, \Phi_{j}^{1}\right\}_{j=1, \ldots, N} \in\left(H^{1}(\Omega) \times L^{2}\left(\Omega ; H_{p e r}^{1}(Y)\right)\right)^{N}
\end{gathered}
$$

be a limit from Theorem 2 . Then it satisfies the two-scale two-pressures homogenized problem 


$$
\begin{gathered}
-\Delta_{y} \mathbf{v}^{0}(x, y)+\nabla_{y} p^{1}(x, y)=-\nabla_{x} p^{0}(x)-\mathbf{f}^{*}(x) \\
+\sum_{j=1}^{N} z_{j} n_{j}^{0}(y)\left(\nabla_{x} \Phi_{j}^{0}(x)+\nabla_{y} \Phi_{j}^{1}(x, y)+\mathbf{E}^{*}(x)\right) \text { in } \Omega \times Y_{F}, \\
\operatorname{div}_{y} \mathbf{v}^{0}(x, y)=0 \text { in } \Omega \times Y_{F}, \quad \mathbf{v}^{0}(x, y)=0 \text { on } \Omega \times S, \\
\operatorname{div}_{x}\left(\int_{Y_{F}} \mathbf{v}^{0}(x, y) d y\right)=0 \text { in } \Omega, \\
-\operatorname{div}_{y}\left(n_{i}^{0}(y)\left(\nabla_{y} \Phi_{i}^{1}(x, y)+\nabla_{x} \Phi_{i}^{0}(x)+\mathbf{E}^{*}(x)+\frac{P e_{i}}{z_{i}} \mathbf{v}^{0}(x, y)\right)\right)=0 \\
\left(\nabla_{y} \Phi_{i}^{1}+\nabla_{x} \Phi_{i}^{0}+\mathbf{E}^{*}\right) \cdot v(y)=0 \text { on } \Omega \times S, \quad i=1, \ldots, N, \\
-d i v_{x} \int_{Y_{F}} n_{i}^{0}(y)\left(\nabla_{y} \Phi_{i}^{1}(x, y)+\nabla_{x} \Phi_{i}^{0}(x)+\mathbf{E}^{*}(x)+\right. \\
\left.\frac{P e_{i}}{z_{i}} \mathbf{v}^{0}(x, y)\right) \text { dy }=0 \quad \text { in } \Omega, \quad i=1, \ldots, N, \\
\Phi_{i}^{0}, \int_{Y_{F}} \mathbf{v}^{0} d y \text { and } p^{0} \text { being } \Omega \text {-periodic in } x,
\end{gathered}
$$

with periodic boundary conditions on the unit cell $Y_{F}$ for all functions depending on $y$ and $S=\partial Y_{S} \backslash \partial Y$.

Remark 17. The limit problem features two incompressibility constraints (160) and (161) which are exactly dual to the two pressures $p^{0}(x)$ and $p^{1}(x, y)$ which are their corresponding Lagrange multipliers. Remark that equations (159), (160) and (162) are just the leading order terms in the ansatz of the original equations. On the other hand, equations (161) and (164) are averages on the unit cell $Y_{F}$ of the next order terms in the ansatz. For example, (161) is deduced from

$$
\operatorname{div}_{y} \mathbf{v}^{1}(x, y)+\operatorname{div}_{x} \mathbf{v}^{0}(x, y)=0 \text { in } \Omega \times Y_{F}
$$

by averaging on $Y_{F}$, recalling that $\mathbf{v}^{1}(x, y)=0$ on $\Omega \times S$.

The detailed proof of convergence and the derivation of the homogenized system corresponds to Theorem 1 in [10]. The limiting procedure gives us the variational form of problem (159)-(165) and it deserves to be recalled here in other to prove the well-posedness of the two-scale homogenized problem.

Following [7], we introduce the functional space for the velocities

$$
V=\left\{\mathbf{v}^{0}(x, y) \in L_{p e r}^{2}\left(\Omega ; H_{p e r}^{1}\left(Y_{F}\right)^{3}\right) \text { satisfying }(160)-(161)\right\},
$$

which is known to be orthogonal in $L_{p e r}^{2}\left(\Omega ; H_{p e r}^{1}\left(Y_{F}\right)^{3}\right)$ to the space of gradients of the form $\nabla_{x} q(x)+\nabla_{y} q_{1}(x, y)$ with $q(x) \in H_{p e r}^{1}(\Omega) / \mathbb{R}$ and $q_{1}(x, y) \in$ $L_{\text {per }}^{2}\left(\Omega ; L_{\text {per }}^{2}\left(Y_{F}\right) / \mathbb{R}\right)$. We define the functional space

$$
X=V \times H_{\text {per }}^{1}(\Omega) / \mathbb{R} \times L_{\text {per }}^{2}\left(\Omega ; H_{\text {per }}^{1}\left(Y_{F}\right)^{d} / \mathbb{R}\right)
$$


and the variational formulation of (159)-(165) is to find $\left(\mathbf{v}^{0},\left\{\Phi_{j}^{0}, \Phi_{j}^{1}\right\}\right) \in X$ such that, for any test functions $\left(\mathbf{v},\left\{\phi_{j}^{0}, \phi_{j}^{1}\right\}\right) \in X$,

$$
a\left(\left(\mathbf{v}^{0},\left\{\Phi_{j}^{0}, \Phi_{j}^{1}\right\}\right),\left(\mathbf{v},\left\{\phi_{j}^{0}, \phi_{j}^{1}\right\}\right)\right)=\left\langle\mathscr{L},\left(\mathbf{v},\left\{\phi_{j}^{0}, \phi_{j}^{1}\right\}\right)\right\rangle,
$$

where the bilinear form $a$ and the linear form $\mathscr{L}$ are defined by

$$
\begin{gathered}
a\left(\left(\mathbf{v}^{0},\left\{\Phi_{j}^{0}, \Phi_{j}^{1}\right\}\right),\left(\mathbf{v},\left\{\phi_{j}^{0}, \phi_{j}^{1}\right\}\right)\right):=\int_{\Omega} \int_{Y_{F}} \nabla_{y} \mathbf{v}^{0}: \nabla \mathbf{v} d x d y \\
+\sum_{i=1}^{N} \frac{z_{i}^{2}}{\mathrm{Pe}_{i}} \int_{\Omega} \int_{Y_{F}} n_{i}^{0}\left(\nabla_{x} \Phi_{i}^{0}+\nabla_{y} \Phi_{i}^{1}\right) \cdot\left(\nabla_{x} \phi_{i}^{0}+\nabla_{y} \phi_{i}^{1}\right) d x d y \\
+\sum_{j=1}^{N} z_{j} \int_{\Omega} \int_{Y_{F}} n_{j}^{0}\left(\mathbf{v}^{0} \cdot\left(\nabla_{x} \phi_{j}^{0}+\nabla_{y} \phi_{j}^{1}\right)-\mathbf{v} \cdot\left(\nabla_{x} \Phi_{j}^{0}+\nabla_{y} \Phi_{j}^{1}\right)\right) d x d y
\end{gathered}
$$

and

$$
\begin{gathered}
<\mathscr{L},\left(\mathbf{v},\left\{\phi_{j}\right\}\right)>:=\sum_{j=1}^{N} z_{j} \int_{\Omega} \int_{Y_{F}} n_{j}^{0} \mathbf{E}^{*} \cdot \mathbf{v} d x d y-\int_{\Omega} \int_{Y_{F}} \mathbf{f}^{*} \cdot \mathbf{v} d x d y \\
-\sum_{i=1}^{N} \frac{z_{i}^{2}}{\mathrm{Pe}_{i}} \int_{\Omega} \int_{Y_{F}} n_{i}^{0} \mathbf{E}^{*} \cdot\left(\nabla_{x} \phi_{i}^{0}+\nabla_{y} \phi_{i}^{1}\right) d x d y
\end{gathered}
$$

We apply the Lax-Milgram lemma to prove the existence and uniqueness of the solution in $X$ of (166). The only point which requires to be checked is the coercivity of the bilinear form. We take $\mathbf{v}=\mathbf{v}^{0}, \phi_{j}^{0}=\Phi_{j}^{0}$ and $\phi_{j}^{1}=\Phi_{j}^{1}$ as the test functions in (166).

Using the incompressibility constraints (161) and the anti-symmetry of the third integral in (167), we obtain the quadratic form

$$
\begin{gathered}
a\left(\left(\mathbf{v}^{0},\left\{\Phi_{j}^{0}, \Phi_{j}^{1}\right\}\right),\left(\mathbf{v}^{0},\left\{\Phi_{j}^{0}, \Phi_{j}^{1}\right\}\right)\right)=\int_{\Omega \times Y_{F}}\left|\nabla_{y} \mathbf{v}^{0}(x, y)\right|^{2} d x d y+ \\
\sum_{j=1}^{N} \frac{z_{j}^{2}}{\operatorname{Pe}_{j}} \int_{\Omega \times Y_{F}} n_{j}^{0}(y)\left|\nabla_{x} \Phi_{j}^{0}(x)+\nabla_{y} \Phi_{j}^{1}(x, y)\right|^{2} d x d y .
\end{gathered}
$$

Recalling from Lemma 5 that $n_{j}^{0}(y) \geq C>0$ in $Y_{F}$, it is easy to check that each term in the sum on the second line of (168) is bounded from below by

$$
C\left(\int_{\Omega}\left|\nabla_{x} \Phi_{j}^{0}(x)\right|^{2} d x+\int_{\Omega \times Y_{F}}\left|\nabla_{y} \Phi_{j}^{1}(x, y)\right|^{2} d x d y\right),
$$

which proves that the bilinear form is $V$-elliptic.

Hence we have proved

Theorem 4. Problem (159)-(165) has a unique solution 


$$
\begin{gathered}
\left(\mathbf{v}^{0}, p^{0}\right) \in L^{2}\left(\Omega ; H_{p e r}^{1}(Y)^{3}\right) \times L_{0}^{2}(\Omega) \quad \text { and } \\
\left\{\Phi_{j}^{0}, \Phi_{j}^{1}\right\}_{j=1, \ldots, N} \in\left(H^{1}(\Omega) \times L^{2}\left(\Omega ; H_{p e r}^{1}(Y)\right)\right)^{N} .
\end{gathered}
$$

and whole sequence $\left(\mathbf{v}^{\varepsilon}, \tilde{P}^{\varepsilon},\left\{\Phi_{j}^{\varepsilon}\right\}\right)$ converges towards it.

\subsection{The separation of the fast and the slow scales and the Onsager relations}

From the point of view of applications, it is important to extract from (159)-(165) the macroscopic homogenized problem. Obviously, it requires to separate the fast and slow scale. It was undertaken by Looker and Carnie in [48] and further improved in [10] and [11].

The main idea is to recognize in the two-scale homogenized problem (159)(165) that there are two different macroscopic fluxes, namely $\left(\nabla_{x} p^{0}(x)+\mathbf{f}^{*}(x)\right)$ and $\left\{\nabla_{x} \Phi_{j}^{0}(x)+\mathbf{E}^{*}(x)\right\}_{1 \leq j \leq N}$. Therefore we introduce two family of cell problems, indexed by $k \in\{1,2,3\}$ for each component of these fluxes. We denote by $\left\{\mathbf{e}^{k}\right\}_{1 \leq k \leq 3}$ the canonical basis of $\mathbb{R}^{3}$.

The first cell problem, corresponding to the macroscopic pressure gradient, is

$$
\begin{gathered}
-\Delta_{y} \mathbf{v}^{0, k}(y)+\nabla_{y} \pi^{0, k}(y)=\mathbf{e}^{k}+\sum_{j=1}^{N} z_{j} n_{j}^{0}(y) \nabla_{y} \theta_{j}^{0, k}(y) \text { in } Y_{F} \\
\operatorname{div}_{y} \mathbf{v}^{0, k}(y)=0 \quad \text { in } Y_{F}, \quad \mathbf{v}^{0, k}(y)=0 \text { on } S, \\
-\operatorname{div}_{y}\left(n_{i}^{0}(y)\left(\nabla_{y} \theta_{i}^{0, k}(y)+\frac{\operatorname{Pe}_{i}}{z_{i}} \mathbf{v}^{0, k}(y)\right)\right)=0 \text { in } Y_{F} \\
\nabla_{y} \theta_{i}^{0, k}(y) \cdot v=0 \text { on } S .
\end{gathered}
$$

The second cell problem, corresponding to the macroscopic diffusive flux, is for each species $l \in\{1, \ldots, N\}$

$$
\begin{gathered}
-\Delta_{y} \mathbf{v}^{l, k}(y)+\nabla_{y} \pi^{l, k}(y)=\sum_{j=1}^{N} z_{j} n_{j}^{0}(y)\left(\delta_{l j} \mathbf{e}^{k}+\nabla_{y} \theta_{j}^{l, k}(y)\right) \text { in } Y_{F} \\
\operatorname{div}_{y} \mathbf{v}^{l, k}(y)=0 \quad \text { in } Y_{F}, \quad \mathbf{v}^{l, k}(y)=0 \quad \text { on } S, \\
\left.-\operatorname{div}_{y}\left(n_{i}^{0}(y)\left(\delta_{i j} \mathbf{e}^{k}+\nabla_{y} \theta_{j}^{i, k}(y)\right)+\frac{P_{i}}{z_{i}} \mathbf{v}^{i, k}(y)\right)\right)=0 \text { in } Y_{F} \\
\left(\delta_{i j} \mathbf{e}^{k}+\nabla_{y} \theta_{j}^{i, k}(y)\right) \cdot v=0 \text { on } S,
\end{gathered}
$$

where $\delta_{i j}$ is the Kronecker symbol. As usual the cell problems are complemented with periodic boundary conditions.

Then, we can decompose the solution of (159)-(165) as 


$$
\begin{aligned}
\mathbf{v}^{0}(x, y) & =\sum_{k=1}^{3}\left(-\mathbf{v}^{0, k}(y)\left(\frac{\partial p^{0}}{\partial x_{k}}+f_{k}^{*}\right)(x)+\sum_{i=1}^{N} \mathbf{v}^{i, k}(y)\left(E_{k}^{*}+\frac{\partial \Phi_{i}^{0}}{\partial x_{k}}\right)(x)\right) \\
p^{1}(x, y) & =\sum_{k=1}^{3}\left(-\pi^{0, k}(y)\left(\frac{\partial p^{0}}{\partial x_{k}}+f_{k}^{*}\right)(x)+\sum_{i=1}^{N} \pi^{i, k}(y)\left(E_{k}^{*}+\frac{\partial \Phi_{i}^{0}}{\partial x_{k}}\right)(x)\right) \\
\Phi_{j}^{1}(x, y) & =\sum_{k=1}^{3}\left(-\theta_{j}^{0, k}(y)\left(\frac{\partial p^{0}}{\partial x_{k}}+f_{k}^{*}\right)(x)+\sum_{i=1}^{N} \theta_{j}^{i, k}(y)\left(E_{k}^{*}+\frac{\partial \Phi_{i}^{0}}{\partial x_{k}}\right)(x)\right) .
\end{aligned}
$$

We average (177)-(179) in order to get a purely macroscopic homogenized problem. We define the homogenized quantities: first, the electrochemical potential

$$
\mu_{j}(x)=-z_{j}\left(\Phi_{j}^{0}(x)+\Psi^{e x t, *}(x)\right),
$$

then, the ionic flux of the $j$ th species

$$
\mathbf{j}_{j}(x)=\frac{1}{\left|Y_{F}\right|} \int_{Y_{F}} n_{j}^{0}(y)\left(\frac{z_{j}}{\operatorname{Pe}_{j}}\left(\nabla_{y} \Phi_{l}^{1}(x, y)+\nabla_{x} \Phi_{l}^{0}(x)+\mathbf{E}^{*}(x)\right)+\mathbf{v}^{0}\right) d y,
$$

and finally the filtration velocity

$$
\mathbf{v}(x)=\frac{1}{\left|Y_{F}\right|} \int_{Y_{F}} \mathbf{v}^{0}(x, y) d y .
$$

From (177)-(179) we deduce the homogenized or upscaled equations for the above effective fields.

Proposition 16. Introducing the flux $\mathscr{J}(x)=\left(\mathbf{v},\left\{\mathbf{j}_{j}\right\}_{1 \leq j \leq N}\right)$ and the gradient $\mathscr{F}(x)=$ $\left(\nabla_{x} p^{0},\left\{\nabla_{x} \mu_{j}\right\}_{1 \leq j \leq N}\right)$, the macroscopic equations are

$$
\begin{gathered}
d i v_{x} \mathscr{J}=0 \quad \text { in } \Omega, \\
\mathscr{J}=-\mathscr{M} \mathscr{F}-\mathscr{M}\left(\mathbf{f}^{*},\{0\}\right),
\end{gathered}
$$

with a homogenized tensor $\mathscr{M}$ defined by

$$
\mathscr{M}=\left(\begin{array}{cccc}
\mathbb{K} & \frac{\mathbb{J}_{1}}{z_{1}} & \cdots & \frac{\mathbb{J}_{N}}{z_{N}} \\
\mathbb{L}_{1} & \frac{\mathbb{D}_{11}}{z_{1}} & \cdots & \frac{\mathbb{D}_{1 N}}{z_{N}} \\
\vdots & \vdots & \ddots & \vdots \\
\mathbb{L}_{N} & \frac{\mathbb{D}_{N 1}}{z_{1}} & \cdots & \frac{\mathbb{D}_{N N}}{z_{N}}
\end{array}\right),
$$

and complemented with periodic boundary conditions for $p^{0}$ and $\left\{\Phi_{j}^{0}\right\}_{1 \leq j \leq N}$. The matrices $\mathbb{J}_{i}, \mathbb{K}, \mathbb{D}_{j i}$ and $\mathbb{L}_{j}$ are defined by their entries 


$$
\begin{gathered}
\left\{\mathbb{J}_{i}\right\}_{l k}=\frac{1}{\left|Y_{F}\right|} \int_{Y_{F}} \mathbf{v}^{i, k}(y) \cdot \mathbf{e}^{l} d y, \\
\{\mathbb{K}\}_{l k}=\frac{1}{\left|Y_{F}\right|} \int_{Y_{F}} \mathbf{v}^{0, k}(y) \cdot \mathbf{e}^{l} d y, \\
\left\{\mathbb{D}_{j i}\right\}_{l k}=\frac{1}{\left|Y_{F}\right|} \int_{Y_{F}} n_{j}^{0}(y)\left(\mathbf{v}^{i, k}(y)+\frac{z_{j}}{P e_{j}}\left(\delta_{i j} \mathbf{e}^{k}+\nabla_{y} \theta_{j}^{i, k}(y)\right)\right) \cdot \mathbf{e}^{l} d y, \\
\left\{\mathbb{L}_{j}\right\}_{l k}=\frac{1}{\left|Y_{F}\right|} \int_{Y_{F}} n_{j}^{0}(y)\left(\mathbf{v}^{0, k}(y)+\frac{z_{j}}{P e_{j}} \nabla_{y} \theta_{j}^{0, k}(y)\right) \cdot \mathbf{e}^{l} d y .
\end{gathered}
$$

Furthermore, $\mathscr{M}$ is symmetric positive definite, which implies that the homogenized equations (183)-(184) have a unique solution.

Remark 18. The symmetry of $\mathscr{M}$ is equivalent to the famous Onsager's reciprocal relations. The symmetry of the tensor $\mathscr{M}$ was proved in [48] and its positive definiteness in [10].

Proof. The conservation law (183) is just a rewriting of (161) and (164). The constitutive equation (184) is an immediate consequence of the definitions (181) and (182) of the homogenized fluxes, taking into account the decomposition (177)-(179).

We now prove that $\mathscr{M}$ is positive definite. For any vectors $\lambda^{0},\left\{\lambda^{i}\right\}_{1 \leq i \leq N} \in \mathbb{R}^{3}$ let us introduce the following linear combinations of the cell solutions

$$
\mathbf{v}^{\lambda}=\sum_{k=1}^{3}\left(\lambda_{k}^{0} \mathbf{v}^{0, k}+\sum_{i=1}^{N} \lambda_{k}^{i} \mathbf{v}^{i, k}\right), \quad \theta_{j}^{\lambda}=\sum_{k=1}^{3}\left(\lambda_{k}^{0} \theta_{j}^{0, k}+\sum_{i=1}^{N} \lambda_{k}^{i} \theta_{j}^{i, k}\right),
$$

which satisfy

$$
\begin{gathered}
-\Delta_{y} \mathbf{v}^{\lambda}(y)+\nabla_{y} \pi^{\lambda}(y)=\lambda^{0}+\sum_{j=1}^{N} z_{j} n_{j}^{0}(y)\left(\lambda^{j}+\nabla_{y} \theta_{j}^{\lambda}(y)\right) \text { in } Y_{F} \\
\operatorname{div}_{y} \mathbf{v}^{\lambda}(y)=0 \quad \text { in } Y_{F}, \quad \mathbf{v}^{\lambda}(y)=0 \quad \text { on } S \\
-\operatorname{div}_{y}\left(n_{i}^{0}(y)\left(z_{i}\left(\lambda^{i}+\nabla_{y} \theta_{i}^{\lambda}(y)\right)+\operatorname{Pe}_{i} \mathbf{v}^{\lambda}(y)\right)\right)=0 \text { in } Y_{F} \\
\left(\lambda^{i}+\nabla_{y} \theta_{i}^{\lambda}(y)\right) \cdot v=0 \text { on } S .
\end{gathered}
$$

Multiplying the Stokes equation (187) by $\mathbf{v}^{\lambda}$, the convection-diffusion equation (189) by $\theta_{j}^{\lambda}$ and summing up, we obtain 


$$
\begin{gathered}
\int_{Y_{F}}\left(\left|\nabla_{y} \mathbf{v}^{\lambda}(y)\right|^{2}+\sum_{i=1}^{N} \frac{z_{i}^{2}}{\mathrm{Pe}_{i}} n_{i}^{0}(y)\left(\nabla_{y} \theta_{i}^{\lambda}(y)+\lambda^{i}\right) \cdot\left(\nabla_{y} \theta_{i}^{\lambda}(y)+\lambda^{i}\right)\right) d y \\
=\int_{Y_{F}} \lambda^{0} \cdot \mathbf{v}^{\lambda} d y+\sum_{i=1}^{N} \int_{Y_{F}} z_{i} n_{i}^{0} \lambda^{i} \cdot \mathbf{v}^{\lambda} d y+\sum_{i=1}^{N} \int_{Y_{F}} \frac{z_{i}^{2}}{\mathrm{Pe}_{i}} n_{i}^{0}\left(\nabla_{y} \theta_{i}^{\lambda}+\lambda^{i}\right) \cdot \lambda^{i} d y \\
=\mathbb{K} \lambda^{0} \cdot \lambda^{0}+\sum_{i=1}^{N} \mathbb{J}_{i} \lambda^{i} \cdot \lambda^{0}+\sum_{i, j=1}^{N} z_{i} \lambda^{i} \cdot \mathbb{D}_{i j} \lambda^{j}+\sum_{i=1}^{N} z_{i} \lambda^{i} \cdot \mathbb{L}_{i} \lambda^{0}= \\
\mathscr{M}\left(\lambda^{0},\left\{z_{i} \lambda^{i}\right\}\right)^{T} \cdot\left(\lambda^{0},\left\{z_{i} \lambda^{i}\right\}\right)^{T} .
\end{gathered}
$$

The left hand side of the above equality is positive. This proves the positive definite character of $\mathscr{M}$.

It remains to prove the symmetry of $\mathscr{M}$. For another set of vectors $\tilde{\lambda}^{0},\left\{\tilde{\lambda}^{i}\right\}_{1 \leq i \leq N} \in$ $\mathbb{R}^{3}$, we define $\mathbf{v}^{\tilde{\lambda}}$ and $\theta_{j}^{\tilde{\lambda}}$ by (186). Multiplying the Stokes equation for $\mathbf{v}^{\lambda}$ by $\mathbf{v}^{\tilde{\lambda}}$ and the convection-diffusion equation for $\theta_{j}^{\tilde{\lambda}}$ by $\theta_{j}^{\lambda}$ (note the skew-symmetry of this computation), then adding the two variational formulations yields

$$
\begin{gathered}
\int_{Y_{F}} \nabla_{y} \mathbf{v}^{\lambda} \cdot \nabla_{y} \mathbf{v}^{\tilde{\lambda}} d y+\sum_{i=1}^{N} \int_{Y_{F}} \frac{z_{i}^{2}}{\mathrm{Pe}_{i}} n_{i}^{0} \nabla_{y} \theta_{i}^{\tilde{\lambda}} \cdot \nabla_{y} \theta_{i}^{\lambda} d y= \\
\int_{Y_{F}} \lambda^{0} \cdot \mathbf{v}^{\tilde{\lambda}} d y+\sum_{j=1}^{N} \int_{Y_{F}} z_{j} n_{j}^{0} \lambda^{j} \cdot \mathbf{v}^{\tilde{\lambda}} d y-\sum_{i=1}^{N} \int_{Y_{F}} \frac{z_{i}^{2}}{\mathrm{Pe}_{i}} n_{i}^{0} \tilde{\lambda}^{i} \cdot \nabla_{y} \theta_{i}^{\lambda} d y .
\end{gathered}
$$

Therefore, the left hand side of (191) is symmetric in $\lambda, \tilde{\lambda}$. Exchanging the last term in (191), we deduce by symmetry

$$
\begin{aligned}
& \int_{Y_{F}} \lambda^{0} \cdot \mathbf{v}^{\tilde{\lambda}} d y+\sum_{j=1}^{N} \int_{Y_{F}} z_{j} n_{j}^{0} \lambda^{j} \cdot \mathbf{v}^{\tilde{\lambda}} d y+\sum_{i=1}^{N} \int_{Y_{F}} \frac{z_{i}^{2}}{\mathrm{Pe}_{i}} n_{i}^{0} \lambda^{i} \cdot \nabla_{y} \theta_{i}^{\tilde{\lambda}} d y \\
= & \int_{Y_{F}} \tilde{\lambda}^{0} \cdot \mathbf{v}^{\lambda} d y+\sum_{j=1}^{N} \int_{Y_{F}} z_{j} n_{j}^{0} \tilde{\lambda}^{j} \cdot \mathbf{v}^{\lambda} d y+\sum_{i=1}^{N} \int_{Y_{F}} \frac{z_{i}^{2}}{\mathrm{Pe}_{i}} n_{i}^{0} \tilde{\lambda}^{i} \cdot \nabla_{y} \theta_{i}^{\lambda} d y,
\end{aligned}
$$

which is equivalent to the desired symmetry

$$
\mathscr{M}\left(\tilde{\lambda}^{0},\left\{z_{i} \tilde{\lambda}^{i}\right\}\right)^{T} \cdot\left(\lambda^{0},\left\{z_{i} \lambda^{i}\right\}\right)^{T}=\mathscr{M}\left(\lambda^{0},\left\{z_{i} \lambda^{i}\right\}\right)^{T} \cdot\left(\tilde{\lambda}^{0},\left\{z_{i} \tilde{\lambda}^{i}\right\}\right)^{T} .
$$

The norm-closeness of the solution to the homogenized problem, to the solution of the original problem is given by the following result.

Theorem 5. ([10]) Let $\left(p^{0},\left\{\Phi_{j}^{0}\right\}_{1 \leq j \leq N}\right)$ be defined by (183)-(184). Let $\mathbf{v}^{0}$ be given by (177) and $\left\{\Phi_{j}^{1}\right\}_{1 \leq j \leq N}$ by (179). Then in the limit $\varepsilon \rightarrow 0$ we have

$$
\int_{\Omega_{\varepsilon}}\left(\left|\mathbf{v}^{\varepsilon}(x)-\mathbf{v}^{0}\left(x, \frac{x}{\varepsilon}\right)\right|^{2}+\left|\tilde{P}^{\varepsilon}(x)-p^{0}(x)\right|^{2}\right) d x \rightarrow 0
$$

and 


$$
\int_{\Omega_{\varepsilon}}\left|\nabla\left(\Phi_{j}^{\varepsilon}(x)-\Phi_{j}^{0}(x)-\varepsilon \Phi_{j}^{1}\left(x, \frac{x}{\varepsilon}\right)\right)\right|^{2} d x \rightarrow 0 .
$$

Acknowledgements This research was partially supported by the MOCOMIPOC project (Modélisation multiéchelles des écoulements complexes en présence de gaz dans les milieux chargés) from the NEEDS program (Projet fédérateur Milieux Poreux MIPOR), part of CNRS, France and by the LABEX MILYON (ANR-10-LABX-0070) of Université de Lyon, within the program "Investissements d'Avenir" (ANR-11-IDEX-0007) operated by the French National Research Agency (ANR).

\section{References}

1. Acerbi, E., Chiadò Piat, V., Dal Maso, G., Percivale, D. : An extension theorem from connected sets, and homogenization in general periodic domains, Nonlinear Anal., TMA, 18, 481 - 496 (1992).

2. Adler, P.M., Mityushev, V.: Effective medium approximation and exact formulae for electrokinetic phenomena in porous media, J. Phys. A: Math. Gen. 36 , 391-404 (2003).

3. Adler, P.M. : Macroscopic electroosmotic coupling coefficient in random porous media, Math. Geol. 33(1), 63-93 (2001).

4. Allaire, G. : Homogenization of the Stokes Flow in a Connected Porous Medium, Asymptotic Analysis, 2 , 203-222 (1989).

5. Allaire, G.: Homogenization and two-scale convergence. SIAM J. Math. Anal., 23 , 1482 $1518(1992)$

6. Allaire, G., A. Damlamian, A., Hornung, U.: Two-scale convergence on periodic surfaces and applications. In: Bourgeat, A., Carasso, C., Luckhaus, S., Mikelić, A. (eds) Mathematical Modelling of Flow through Porous Media, pp. 15-25. World Scientific, Singapore (1995).

7. Allaire, G. : One-Phase Newtonian Flow. In Hornung, U. (ed) Homogenization and Porous Media, pp. 45-68. Springer, New-York (1997).

8. Allaire, G., Brizzi, R., Mikelić, A., Piatnitski, A.: Two-scale expansion with drift approach to the Taylor dispersion for reactive transport through porous media. Chemical Engineering Science, $\mathbf{6 5}, 2292-2300$ (2010).

9. Allaire, G., Mikelić, A., Piatnitski, A.: Homogenization approach to the dispersion theory for reactive transport through porous media. SIAM J. Math. Anal., 42, 125-144 (2010).

10. Allaire, G., Mikelić, A., Piatnitski, A.: Homogenization of The Linearized Ionic Transport Equations in Rigid Periodic Porous Media, Journal of Mathematical Physics, 51, 123103 (2010). Erratum in the same journal, 52, 063701 (2011).

11. Allaire, G., Brizzi, R., Dufrêche, J.-F., Mikelić, A. Piatnitski, A. : Ion transport in porous media: derivation of the macroscopic equations using upscaling and properties of the effective coefficients, Comp. Geosci., 17, 479-495 (2013).

12. Allaire, G., Dufrêche, J.-F., Mikelić, A., Piatnitski, A.: Asymptotic analysis of the PoissonBoltzmann equation describing electrokinetics in porous media, Nonlinearity, 26, 881-910 (2013).

13. Allaire, G., Brizzi, R., Dufrêche, J.-F., Mikelić, A., Piatnitski, A. : Role of non-ideality for the ion transport in porous media: derivation of the macroscopic equations using upscaling. Phys. D, 282 , 39-60 (2014).

14. Allaire, G., Bernard, O., Dufrêche, J.-F., Mikelić, A. : Ion transport through deformable porous media: derivation of the macroscopic equations using upscalin., 2015, < hal01215457>, accepted for publication in Comp. Appl. Math., 2016, doi:10.1007/s40314-0160321-0.

15. Auriault, J. L., Strzelecki, T.: On the electro-osmotic flow in a saturated porous medium, Int. J. Engng Sci. 19, 915-928 (1981). 
16. Beliaev, A. Yu., Kozlov S. M. : Darcy equation for random porous media, Comm. Pure Appl. Math. , 49, 1-34 (1995).

17. Baranger, J., Najib, K. : Analyse numérique des écoulements quasi-newtoniens dont la viscosité obéit à la loi puissance ou la loi de Carreau, Numer. Math. 58, 3549 (1990).

18. Bird, R.B. , Stewart, W. E. N. Lightfoot, E. N. : Transport Phenomena. Wiley and Sons, New York (1960).

19. Bird, R.B., Armstrong R.C., Hassager, O. : Dynamics of Polymeric Liquids, Vol.1, Fluid Mechanics. Wiley, New York, (1987).

20. Bourgeat, A., Mikelić, A. : Note on the Homogenization of Bingham Flow through Porous Medium. Journal de Mathématiques Pures et Appliquées, 72, 405-414 (1993).

21. Bourgeat, A., Mikelić, A., Tapiero, R. : Dérivation des équations moyennées décrivant un écoulement non newtonien dans un domaine de faible épaisseur, C. R. Acad. Sci. Paris, Sér. I 316 (1993), 965970.

22. Bourgeat, A., Mikelić, A. : Homogenization of the Non-Newtonian Flow through Porous Medium, Nonlinear Anal., Theory Methods Appl., 26, 1221 - 1253 (1996).

23. Bourgeat, A., Gipouloux, O., Marusic-Paloka, E. : Filtration law for polymer flow through porous media. Multiscale Modeling Simulation, 1, 432-457 (2003).

24. Brezis, H.: Functional analysis, Sobolev spaces and partial differential equations. Springer Science andBusiness Media, New York (2010).

25. Bulíček, M. , Gwiazda, P. , Málek, J., Świerczewska-Gwiazda, A.: On steady flows of incompressible fluids with implicit power-law-like rheology. Advances in Calculus of Variations 2, 109136 (2009).

26. Cattabriga, L. : Su un problema al contorno relativo al sistema di equazioni di Stokes. Rendiconti del Seminario Matematico della Universit di Padova, 31, 308-340 (1961).

27. Chan, D. Y., Horn, R.G. : The drainage of thin liquid films between solid surfaces, J. Chem. Phys. 83, 5311-5325 (1985).

28. Christopher, R. H., Middleman, S. : Power-Law Flow Through Porous Media, Ind. Eng. Cheni. Fund., 4, 422 (1965).

29. Cioranescu, D., Donato, P.: An Introduction to Homogenization. Oxford University Press, Oxford (2000).

30. Coelho, D., Shapiro, M., Thovert, J.-F. , Adler, P. M. : Electro-osmotic phenomena in porous media, J. Colloid Interface Sci. 181, 169-90 (1996).

31. Dufrêche, J.-F. , Bernard, O. , Durand-Vidal, S. , Turq, P. : Analytical Theories of Transport in Concentrated Electrolyte Solutions from the MSA, J. Phys. Chem. B 109 , 9873 (2005).

32. Dufrêche, J.-F., Marry, V. , Malikova, N. , Turq, P., : Molecular hydrodynamics for electroosmosis in clays: from Kubo to Smoluchowski, J. Mol. Liq. 118, 145 (2005).

33. Duvaut G., Lions J.L.: Inequalities in Mechanics and Physics. Springer-Verlag, Heidelberg (1976)

34. Ekeland, I., Temam, R. : Analyse Convexe et Problèmes Variationnels (Gauthier-Villars, 1973).

35. Ene, H. I. , Sanchez-Palencia, E. : Equations et phénomènes de surface pour l'écoulement dans un modèle de milieu poreux. J. Mécan., 14 , 73-108 (1975).

36. Ern, A. , Joubaud, R. , Lelièvre, T.: Mathematical study of non-ideal electrostatic correlations in equilibrium electrolytes, Nonlinearity, 25 1635-1652 (2012).

37. Gipouloux, O., Zine, A.M.: Computation of the filtration laws through porous media for a non-Newtonian fluid obeying the power law, Computational Geosciences 1, 127-153 (1997).

38. Gupta, A.K. , Coelho, D. , Adler, P.M.: Electroosmosis in porous solids for high zeta potentials, Journal of Colloid and Interface Science 303, 593-603 (2006).

39. Hornung, U., ed. : Homogenization and Porous Media, Interdisciplinary Applied Mathematics Series 6 Springer, New York, (1997)

40. Jikov, V.V., Kozlov, S., Oleinik, O. : Homogenization of Differential Operators and Integral Functionals. Springer Verlag, New York, (1994)

41. Kaloušek, M. : Homogenization of incompressible generalized Stokes flows through a porous medium. Nonlinear Analysis: Theory, Methods and Applications, 136, 1-39 (2016). 
42. Karniadakis, G., Beskok, A., Aluru, N. : Microflows and Nanoflows. Fundamentals and Simulation, Interdisciplinary Applied Mathematics, Vol. 29, Springer, New York (2005).

43. Khuzhayorov, B., Auriault, J.-L., Royer, P. : Derivation of macroscopic filtration law for transient linear viscoelastic fluid flow in porous media. International Journal of Engineering Science 38, 487-504 (2000).

44. Lions, J.L. : Quelques méthodes de résolution des problèmes aux limites non linéaires. Dunod Gauthier-Villars, Paris (1969).

45. Lions, J.L., Sanchez-Palencia, E.: Écoulement d'un fluide viscoplastique de Bingham dans un milieu poreux. J. Math. pures et appl. 60, 341-360 (1981).

46. Lipton, R., Avellaneda, M. : A Darcy Law for Slow Viscous Flow Past a Stationary Array of Bubbles. Proc. Royal Soc. Edinburgh 114A, 71-79 (1990).

47. Looker, J. R. : Semilinear elliptic Neumann problems and rapid growth in the nonlinearity, Bull. Austral. Math. Soc., Vol. 74, 161-175 (2006).

48. Looker, J. R. , Carnie, S. L. : Homogenization of the ionic transport equations in periodic porous media, Transp. Porous Media 65, 107-131 (2006).

49. Lukkassen, D., Nguetseng, G., Wall, P. : Two-scale convergence. International Journal of Pure and Applied Mathematical Sciences, 2, 35-86 (2002)

50. Marciniak-Czochra, A., Ptashnyk, M. : Derivation of a macroscopic receptor-based model using homogenization techniques. SIAM Journal on Mathematical Analysis, 40, 215-237 (2008).

51. Marino, S., Shapiro, M., Adler, P. M. : Coupled transports in heterogeneous media, J. Colloid Interface Sci. 243, 391-419 (2001).

52. Marry, V., Dufrêche, J.-F., Jardat, M., Turq, P. : Equilibrium and electrokinetic phenomena in charged porous media from microscopic and mesoscopic models: electro-osmosis in montmorillonite, Mol. Phys. 101, 3111 (2005).

53. Mei, C. C. , Vernescu, B. : Homogenization Methods for Multiscale Mechanics. World Scientific Publishing Company, Singapour (2010).

54. Marušić-Paloka, E., Piatnitski, A.: Homogenization of a nonlinear convection-diffusion equation with rapidly oscillating coefficients and strong convection. Journal of London Math. Soc., Vol. 72, 391-409 (2005).

55. Mikelić, A., Tapiero, R. : Mathematical derivation of the power law describing polymer flow through a thin slab. Math. Modelling Numer. Anal. 29, 322 (1995).

56. Mikelić, A. : Non-Newtonian Flow. In Hornung, U. (ed) Homogenization and Porous Media, pp. 69-95. Springer, New-York (1997).

57. Moyne, C., Murad, M. : Electro-chemo-mechanical couplings in swelling clays derived from a micro/macro-homogenization procedure, Int. J. Solids Structures 39, 6159-6190 (2002).

58. Moyne, C., Murad, M. : Macroscopic behavior of swelling porous media derived from micromechanical analysis, Transport Porous Media 50, 127-151 (2003).

59. Moyne, C., Murad, M. : A Two-scale model for coupled electro-chemomechanical phenomena and Onsager's reciprocity relations in expansive clays: I Homogenization analysis, Transport Porous Media 62, 333-380 (2006).

60. Moyne, C., Murad, M. : A two-scale model for coupled electro-chemo-mechanical phenomena and Onsager's reciprocity relations in expansive clays: II. Computational validation, Transp. Porous Media 63(1), 13-56 (2006).

61. Moyne, C., Murad, M. : A dual-porosity model for ionic solute transport in expansive clays, Comput Geosci 12, 47-82 (2008).

62. Neuss-Radu, M. : Some extensions of two-scale convergence. C. R. Acad. Sci. Paris Sér. I Math. 322 (1996), no. 9, p. 899-904.

63. Nguetseng, G. : A General Convergence Result for a Functional Related to the Theory of Homogenization. SIAM J. Math. Analysis, 20, 608-623 (1989).

64. O'Brien, R. W., White, L. R. : Electrophoretic mobility of a spherical colloidal particle, J. Chem. Soc., Faraday Trans. 2 74(2), 1607-1626 (1978).

65. Oleinik, O. A. E., Shamaev, A. S., Yosifian, G. A. : Mathematical problems in elasticity and homogenization. Vol. 2, Elsevier, Amsterdam (2009). 
66. Orgéas, L., Idris, Z., Geindreau, C., Bloch, J.-F., Auriault, J.-L. : Modelling the flow of powerlaw fluids through anisotropic porous media at low-pore Reynolds numbe., Chemical Engineering Science, 61, 44904502 (2006).

67. Pavliotis, G. A., Stuart, A.: Multiscale Methods: Averaging and Homogenization. Springer New York (2008).

68. Pearson, J., Tardy, P. : Models for flow of non-Newtonian and complex fluids through porous media. Journal of Non-Newtonian Fluid Mechanics, 102, 447473 (2002).

69. Ray, N. , Eck, Ch., Muntean, A., Knabner, P.: Variable Choices of Scaling in the Homogenization of a Nernst-Planck-Poisson Problem, preprint no. 344, Institut für Angewandte Mathematik, Universitaet Erlangen-Nürnberg (2011).

70. Ray, N., Muntean, A., Knabner, P. : Rigorous homogenization of a Stokes-Nernst-PlanckPoisson system, J. Math. Anal. Appl. 390(1), 374-393 (2012).

71. Rosanne, M., Paszkuta, M., Adler, P. M.: Electrokinetic phenomena in saturated compact clays, Journal of Colloid and Interface Science, 297, 353-364 (2006).

72. Sanchez-Palencia, E.: Non-Homogeneous Media and Vibration Theory. Lecture Notes in Physics 127, Springer Verlag, Heidelberg (1980).

73. Shah, C.B. , Yortsos, Y. C. : Aspects of Flow of Power-Law Fluids in Porous Media, AIChE Journal, 41, 1099-1112 (1995).

74. Shahsavari, S., McKinley, G. H. : Mobility of power-law and Carreau fluids through fibrous media. Physical Review E, 92, 063012 (2015).

75. Shahsavari, S., McKinley, G. H. : Mobility and pore-scale fluid dynamics of rate-dependent yield-stress fluids flowing through fibrous porous media. Journal of Non-Newtonian Fluid Mechanics, 235, 76-82 (2016).

76. Schmuck, M.: Analysis of the NavierStokesNernstPlanckPoisson system. Mathematical Models and Methods in Applied Sciences, 19, 993-1014 (2009)..

77. Schmuck, M. : Modeling And Deriving Porous Media Stokes-Poisson-Nernst-Planck Equations By A Multiple-Scale Approach, Commun. Math. Sci. 9, no. 3, 685-710 (2011).

78. Schmuck, M.: First error bounds for the porous media approximation of the PoissonNernstPlanck equations. ZAMM (Journal of Applied Mathematics and Mechanics/Zeitschrift für Angewandte Mathematik und Mechanik), 92, 304-319 (2012).

79. Schmuck, M. : New porous medium Poisson-Nernst-Planck equations for strongly oscillating electric potentials. Journal of Mathematical Physics, 54, 021504 (2013).

80. Temam, R. : Navier-Stokes Equations. Elsevier, Amsterdam (1984).

81. Wu, Y. S., Pruess, K., Witherspoon, P. A. : Displacement of a Newtonian Fluid by a NonNewtonian Fluid in a Porous Medium, Trans. Porous Media, 6, 115 (1991). 